\title{
The Additivity of the two-dimensional Miller ideal
}

\author{
Dissertation \\ zur Erlangung des Doktorgrades \\ der Mathematisch-Naturwissenschaftlichen Fakultät \\ der Christian-Albrechts-Universität \\ zu Kiel
}

vorgelegt von

Sonja Thiele

Kiel 2005 

The additivity of the two-dimensional Miller ideal

Dissertation, 2005

\section{Sonja Thiele}

Christian-Albrechts-Universität zu Kiel

Mathematisches Seminar

Ludewig-Meyn-Straße 4

D-24098 Kiel

Referent: Prof. Dr. Otmar Spinas

Korreferent:

Tag der mündlichen Prüfung:

Zum Druck genehmigt: Kiel, 



\section{Contents}

$\begin{array}{ll}\text { Introduction } & 7\end{array}$

$\begin{array}{lll}0 & \text { Preliminaries } & 11\end{array}$

1 The consistency of $\operatorname{cov}(\mathcal{M})<\operatorname{add}\left(\mathcal{J}\left(\mathbb{M}^{2}\right)\right) \quad 15$

1.1 An amoeba forcing for $\mathbb{M}^{2} \ldots \ldots \ldots \ldots \ldots$

$1.2 \mathbb{A}\left(\mathbb{M}^{2}\right)$ has the pure decision property $\ldots \ldots \ldots . \ldots 16$

$1.3 \mathbb{A}\left(\mathbb{M}^{2}\right)$ does not add Cohen-reals . . . . . . . . . . 22

1.4 The model . . . . . . . . . . . . . . . . . 27

2 Martin's Axiom and the additivity of $\mathcal{J}\left(\mathbb{M}^{2}\right)$

2.1 Good sequences . . . . . . . . . . . . . . 31

2.2 The proof of the maintheorem . . . . . . . . . . 40

$2.3 \mathrm{MA}(\sigma$-centered $)$ implies Martin's Axiom for $\mathbb{M}^{2} \ldots \ldots . . . .44$

3 Infinite maximal antichains in $(\mathfrak{P}(\omega) / \text { fin })^{n^{*}} \quad 49$

3.1 Definitions and combinatorics . . . . . . . . . . . . . 49

3.2 A finite-dimensional version of Mathias forcing $\ldots \ldots \ldots 52$

3.3 Finding Ramsey ultrafilters . . . . . . . . . . . . . . 54

3.4 The proof of the maintheorem . . . . . . . . 56

$\begin{array}{ll}\text { Bibliography } & 59\end{array}$ 


\section{Introduction}

For several of the classical tree forcings, such as Sacks forcing $\mathbb{S}$, Laver forcing $\mathbb{L}$ and Miller forcing $\mathbb{M}$, there are corresponding $\sigma$-ideals. Already in 1935 Marcewski [Ma] studied the $\sigma$-ideal $\mathcal{J}(\mathbb{S}):=\left\{X \subseteq{ }^{\omega} 2 \mid \forall p \in \mathbb{S} \exists q \in \mathbb{S}(q \leq\right.$ $p \wedge[q] \cap X=\emptyset)\}$, later, for example, Veličković, Judah, Miller and Shelah $[\mathrm{Ve}, \mathrm{JuMiSh}]$ continued this investigation of $\mathcal{J}(\mathbb{S})$. Brendle, Goldstern, Johnson, Judah, Miller, Repický, Shelah and Spinas [Br, JuMiSh, GoReShSp, GoJSp, Sp1] studied the $\sigma$-ideals $J(Q):=\left\{X \subseteq{ }^{\omega} \omega \mid \forall p \in Q \exists q \in Q(q \leq p \wedge[q] \cap X=\right.$ $\emptyset)\}$ for $Q \in\{\mathbb{M}, \mathbb{L}\}$. For every ideal $\mathcal{I}$ one can define the additivity $\operatorname{add}(\mathcal{I})$ and the covering number $\operatorname{cov}(\mathcal{I})$ of $\mathcal{I}$. The additivity is the minimal cardinality of a subset of the ideal whose union is not in the ideal, and the covering number is the least cardinality of a subset of the ideal whose union is the whole set, on which the ideal is defined. Clearly, the following inequalities hold for every $\sigma$-ideal $\mathcal{I}$ on ${ }^{\omega} 2$ or ${ }^{\omega} \omega$ :

$$
\omega_{1} \leq \operatorname{add}(\mathcal{I}) \leq \operatorname{cov}(\mathcal{I}) \leq 2^{\omega}
$$

and a typical question is whether any of the above inequalities could consistently be strict. Judah, Miller and Shelah built a model for $\operatorname{add}(\mathcal{J}(\mathbb{S}))<\operatorname{cov}(\mathcal{J}(\mathbb{S}))$ and Goldstern, Repický, Shelah and Spinas built models for $\operatorname{add}(J(Q))<$ $\operatorname{cov}(J(Q))$ for $Q \in\{\mathbb{M}, \mathbb{L}\}$. In [Sp1, Sp2, Sp3] Spinas started to develop a combinatorial theory for the two-dimensional Miller forcing $\mathbb{M}^{2}$, where $\mathbb{M}^{n}$ for $n \in \omega \backslash\{0\}$ consists of all $n$-tupels of superperfect trees and carries the coordinatewise ordering. It turned out that $\mathbb{M}^{2}$ is much closer to $\mathbb{M}$ as it is to $\mathbb{M}^{3}$. On the one hand, for example, both, $\mathbb{M}$ and $\mathbb{M}^{2}$ do not add a Cohen-real (see $[\mathrm{Mi}, \mathrm{Sp} 2]$ ), while on the other hand, by an unpublished result of Shelah, $\mathbb{M}^{3}$ does add a Cohen-real.

Jossen and Spinas [JoSp] started the investigation of the $n$-dimensional ideals

$$
\begin{gathered}
\mathcal{J}\left(Q^{n}\right):=\left\{X \subseteq\left({ }^{\omega} \omega\right)^{n} \mid \forall\left(p_{0}, \ldots, p_{n-1}\right) \in Q^{n} \exists\left(q_{0}, \ldots, q_{n-1}\right) \in Q^{n}\right. \\
\left.\left(\left(q_{0}, \ldots, q_{n-1}\right) \leq\left(p_{0}, \ldots, p_{n-1}\right) \wedge\left(\left[q_{0}\right] \times \ldots \times\left[q_{n-1}\right]\right) \cap X=\emptyset\right)\right\}
\end{gathered}
$$

for $Q \in\{\mathbb{L}, \mathbb{M}\}$. They showed that only the two-dimensional Miller ideal $J\left(\mathbb{M}^{2}\right)$ is a $\sigma$-ideal, but neither the higher dimensional Miller ideals $J\left(\mathbb{M}^{n}\right)$ 
for $n \geq 3$ nor the Laver ideals $J\left(\mathbb{L}^{m}\right)$ for $m \geq 2$ are $\sigma$-ideals, thus the additivity of the higher dimensional ideals is $\omega$. Therefore, it is interesting to pay more attention to $\mathcal{J}\left(\mathbb{M}^{2}\right)$. Analogously to the one-dimensional case, Jossen and Spinas have built a model for $\operatorname{add}\left(J\left(\mathbb{M}^{2}\right)\right)<\operatorname{cov}\left(J\left(\mathbb{M}^{2}\right)\right)$. It is obtained - starting with a model of ZFC and the continuum hypothesis - by a countable support iteration of $\mathbb{M}^{2}$ of length $\omega_{2}$. Since no Cohen-reals are added, $\operatorname{cov}(\mathcal{M})<\operatorname{cov}\left(J\left(\mathbb{M}^{2}\right)\right)$ holds in their model, hence, since the continuum equals $\omega_{2}$, we have $\operatorname{cov}(\mathcal{M})=\operatorname{add}\left(J\left(\mathbb{M}^{2}\right)\right)=\omega_{1}$ there, where $\mathcal{M}$ is the ideal of all meager subsets of ${ }^{\omega} \omega$.

Here we prove the consistency of $\operatorname{cov}(\mathcal{M})<\operatorname{add}\left(J\left(\mathbb{M}^{2}\right)\right)$ (Theorem 1.1.2). The natural forcings to increase the additivity numbers are called amoeba forcings. In general, given any definable tree forcing $Q$, an amoeba forcing $\mathbb{A}\left(Q^{n}\right)$ for $Q^{n}$ is a forcing adding some $\left(p_{0}, \ldots, p_{n-1}\right) \in Q^{n}$ such that every $n$-tupel of branches $\left(x_{0}, \ldots, x_{n-1}\right) \in\left[p_{0}\right] \times \ldots \times\left[p_{n-1}\right]$ is $Q^{n}$-generic. We construct an amoeba forcing $\mathbb{A}\left(\mathbb{M}^{2}\right)$ for $\mathbb{M}^{2}$ that does not add Cohen-reals. Our construction is inspired by the work of Spinas [Sp1], where, in the one-dimensional situation, amoeba forcings for $\mathbb{L}$ and $\mathbb{M}$ have been constructed, which have the Laver property. The Laver property is a combinatorial property ruling out that Cohen-reals are added. By a result of Shelah, the Laver property is preserved under countable support forcing iterations. Our $\mathbb{A}\left(\mathbb{M}^{2}\right)$ will also have the Laver property, hence we can increase $\operatorname{add}\left(\mathcal{J}\left(\mathbb{M}^{2}\right)\right)$ without adding Cohen-reals. Let us remark, that the natural amoeba forcings for $\mathbb{L}, \mathbb{M}$ and $\mathbb{M}^{2}$ that come to one's mind are not suitable for our purpose, as they add lots of Cohen-reals (see [Sp1]).

Similar ideas as in [Sp1] have been used independently in [LoShVe], where implicitly an amoeba forcing for Sacks forcing has been constructed, which does not add Cohen-reals.

Judah, Miller, Shelah and Veličković [JuMiSh, Ve] have independently shown that Martin's Axiom does not imply that $\operatorname{add}(\mathcal{J}(\mathbb{S}))=2^{\omega}$. In contrast to this, it is possible to blow up $\operatorname{add}(\mathcal{J}(\mathbb{L}))$ and $\operatorname{add}(\mathcal{J}(\mathbb{M}))$ by a forcing fulfilling the countable chain condition. In fact, in [JuMiSh] it was shown that Martin's Axiom $\left(t=2^{\omega}\right.$ is enough, where $t$ is the tower number) implies $\operatorname{add}(\mathcal{J}(\mathbb{L}))=2^{\omega}$. The analogous result for $\mathbb{M}$ was claimed as well, but the proof was faulty. It was later corrected in [GoJSp]. Here we show that Martin's Axiom for $\sigma$-centered forcings $(\mathrm{MA}(\sigma$-centered $))$ implies that add $\left(\mathcal{J}\left(\mathbb{M}^{2}\right)\right)=2^{\omega}$ hold (Theorem 2.0.5) by combining the ideas of these two papers with the combinatorial properties of $\mathbb{M}^{2}$. As a corollary it turns out that under the assumption that MA $(\sigma$-centered $)$ is true forcing with $\mathbb{M}^{2}$ does not collapse cardinals. By using the same methods we can prove that MA( $\sigma$-centered $)$ implies Martin's Axiom for the forcing $\mathbb{M}^{2}$ (Theorem 2.3.5).

In chapter 3 we will treat a completely different problem with similar methods, in the sense that carefully chosen finite products of Mathias forcing are used that have the Laver property. In [Ma], Mathias introduced his famous Math- 
ias forcing $M(U)$ restricted to a Ramsey ultrafilter $U$, i.e. $M(U):=\{(s, S) \in$ $[\omega]^{<\omega} \times[\omega]^{\omega} \mid(\max (s)<\min (S)$ or $s=\emptyset)$ and $\left.S \in U\right\}$ and the ordering is defined by $(s, S) \leq(t, T)$ if and only if $s \supseteq t$ and $S \cup(s \backslash t) \subseteq T$. The analysis of this forcing led him, among many other things, to the result that there are no analytical infinite maximal almost disjoint families in $\mathfrak{P}(\omega)$. Note that maximal almost disjoint families in $\mathfrak{P}(\omega)$ correspond to maximal antichains in the Boolean algebra $\mathfrak{P}(\omega)$ /fin. Here we investigate infinite maximal antichains in finite products of $\mathfrak{P}(\omega) /$ fin. Using similar but simpler ideas as in [LoShVe] and [Sp1], Shelah and Spinas [ShSp2] introduced an $n$-dimensional version of Mathias forcing with the Laver property. Combining their analysis of this forcing with some combinatorial facts about infinite maximal antichains in $(\mathfrak{P}(\omega) / \mathrm{fin})^{n}$ we prove that for every $n \in \omega \backslash\{0,1\}$ there are no analytical infinite maximal antichains in $(\mathfrak{P}(\omega) / \text { fin })^{n}$ (Theorem 3.1.1). Curiously, in dimension $\omega$ there exists a perfect (thus closed) partition, as the following example shows: choose $a \in[\omega]^{\omega}$ with $\omega \backslash a \in[\omega]^{\omega}$, too, and consider $\mathcal{A}:=\left\{\left(a_{i}\right)_{i \in \omega} \mid \forall i \in \omega\left(a_{i} \in\{a, \omega \backslash a\}\right)\right\}$.

I would like to thank Otmar Spinas for many helpful discussions and his dedicated supervision of this thesis. 


\section{Chapter 0}

\section{Preliminaries}

We fix our notation: For a set $A$ let $[A]^{<\omega}$ denote the collection of all finite subsets of $A$ and let $[A]^{\omega}$ denote the collection of all countably infinite subsets of $A$. ${ }^{<\omega} A$ denotes the set of all functions $s: n \rightarrow A$ for some $n \in \omega,{ }^{\omega} A$ is the set of all functions $f: \omega \rightarrow A$. For $s \in{ }^{<\omega} A$ for any set $A$ we write $|s|=n$ (the length of $s$ ) if $s: n \rightarrow A$. For $a \in A$ let $s \frown\langle a\rangle$ be the function $s \cup\{(|s|, a)\}$.

Trees: A set $p \subseteq{ }^{<\omega} \omega$ is called a tree if for every $\sigma \in p$ and $\tau \subseteq \sigma$ we have $\tau \in p$. Given a tree $p \subseteq{ }^{<\omega} \omega$, the set of all infinite branches through $p$ is denoted by $[p]$. By $\operatorname{st}(p)$ we denote the stem of $p$, this is the shortest splitnode in $p$. For $\sigma \in p$ let $(p)_{\sigma}$ be the subtree of $p$ consisting of all $\nu \in p$ which are comparable with $\sigma$. For $\sigma \in p$ let $\operatorname{succ}_{p}(\sigma)$ designates the set of all extensions of the form $\sigma^{\frown}\langle n\rangle$, for $n \in \omega$, with $\sigma \frown\langle n\rangle \in p$. Call $\sigma \in p$ a splitnode, if $\left|\operatorname{succ}_{p}(\sigma)\right|>1$, an infinite splitnode, if $\left|\operatorname{succ}_{p}(\sigma)\right|$ is infinite. The set of all infinite splitnodes of a tree $p$ is denoted by $\operatorname{split}(p)$. For $\sigma \in \operatorname{split}(p)$ define $\operatorname{Succ}_{p}(\sigma):=\{\tau \in \operatorname{split}(p) \mid \sigma \varsubsetneqq \tau \wedge \forall \rho(\sigma \varsubsetneqq \rho \varsubsetneqq \tau \Rightarrow \rho \notin \operatorname{split}(p))\}$, the set of all direct successors of $\sigma$ in the tree sense in $\operatorname{split}(p)$. A tree $p \subseteq{ }^{<\omega} \omega$ is called a Laver tree if it has a stem st $(p)$ and for every $\sigma \in p$ with $\sigma \supseteq \operatorname{st}(p)$ we have $\sigma \in \operatorname{split}(p)$. Laver forcing is the set of all Laver trees $\mathbb{L}$ ordered by inclusion. A tree $p \subseteq{ }^{<\omega} \omega$ is called a Miller or superperfect tree, if it has a stem and for every $\sigma \in p$ there exists an extension $\tau \supsetneqq \sigma$ in $p$ which is an infinite splitnode. Let $\mathbb{M}$ denote the set of all superperfect trees $p$ with the additional property, that every splitnode of $p$ is an infinite one. Miller forcing is $\mathbb{M}$ ordered by inclusion, i.e. $p \leq q$ if and only if $p \subseteq q$. Notice that $\mathbb{M}$ is dense in the forcing sense in the set of all superperfect trees. During the whole paper, if we write $p$ is "superperfect" or "Miller" we mean $p \in \mathbb{M}$. Let $\mathbb{M}^{2}$ and more generally $\mathbb{M}^{n}$ denote the set of all $n$-tupels of superperfect trees carrying the coordinatewise ordering. We write $p \leq 0 q$ if $p \leq q$ and additionally we have $\operatorname{st}(p)=\operatorname{st}(q)$ and $\left(p^{\prime}, q^{\prime}\right) \leq 0(p, q)$ if $\left(p^{\prime}, q^{\prime}\right) \leq(p, q)$ and we have that $\operatorname{st}\left(p^{\prime}\right)=\operatorname{st}(p)$ and $\operatorname{st}\left(q^{\prime}\right)=\operatorname{st}(q)$ hold.

A set $S \subseteq{ }^{\omega} \omega$ is called superperfect, if there exists a superperfect tree $p \in$ 
$\mathbb{M}$ with $S=[p]$. For $(p, q) \in \mathbb{M}^{2}$ by $[p] \times \times^{+}[q]$ we denote the upper half of the superperfect rectangle $[p] \times[q]$, i.e. the set of all $(x, y) \in[p] \times[q]$ with $x(|\operatorname{st}(p)|)<y(|\operatorname{st}(q)|)$. Similar, $[p] \times^{-}[q]$ denotes the lower half of $[p] \times[q]$.

We use the following well-ordering $\prec$ of $<\omega \omega$ :

$$
\begin{aligned}
& \sigma \prec \tau: \Leftrightarrow \max \{|\sigma|, \max \operatorname{ran}(\sigma)\}<\max \{|\tau|, \max \operatorname{ran}(\tau)\} \\
& \vee(\max \{|\sigma|, \max \operatorname{ran}(\sigma)\}=\max \{|\tau|, \max \operatorname{ran}(\tau)\} \wedge|\sigma|<|\tau|) \\
& \vee(\max \{|\sigma|, \max \operatorname{ran}(\sigma)\}=\max \{|\tau|, \max \operatorname{ran}(\tau)\} \wedge|\sigma|=|\tau| \\
& \wedge \sigma \text { precedes } \tau \text { lexicographically). }
\end{aligned}
$$

Let $T: \omega \rightarrow{ }^{<\omega} \omega$ be the order preserving enumeration of $\left({ }^{<\omega} \omega, \prec\right)$ and write $\# \rho=n$ if $T(n)=\rho$, so we have $\# \rho \geq \max \{|\rho|, \max \operatorname{ran}(\rho)\}$.

We repeat the following definition and fact of [Sp2]:

Definition 0.0.1 Let $(\sigma, \tau) \in\left({ }^{<\omega} \omega\right)^{2}$ and $(x, y) \in\left({ }^{\omega} \omega\right)^{2}$ with $\sigma \subseteq x$ and $\tau \subseteq y$. We say that $(x, y)$ oscillates infinitely often above $(\sigma, \tau)$ if there exists a strictly increasing sequence $\left(k_{i}\right)_{i \in \omega}$ in $\omega$ such that the following hold for all $n \in \omega:$

$$
\begin{aligned}
& k_{0}=|\tau|, k_{1}>|\sigma| ; \\
& k_{2 n}=\min \left\{i \in \omega \mid y(i)>\# x\left\lceil k_{2 n+1}\right\} ;\right. \\
& k_{2 n+1}=\min \left\{i \in \omega \mid x(i)>\# y\left\lceil k_{2 n+2}\right\} ;\right. \\
& k_{2 n+1}<y\left(k_{2 n}\right)<k_{2 n+2}<x\left(k_{2 n+1}\right)<k_{2 n+3} .
\end{aligned}
$$

The sequence

$$
\left(\sigma, \tau, x\left\lceil k_{1}, y\left\lceil k_{2}, x \uparrow k_{3}, y \uparrow k_{4}, \ldots\right)\right.\right.
$$

is called the type ${ }_{\sigma, \tau}$-sequence of the pair $(x, y)$.

Fact 0.0.2 [Sp2] For every $(p, q) \in \mathbb{M}^{2}$ there exists $\left(p^{\prime}, q^{\prime}\right) \leq 0(p, q)$ such that for every $(x, y) \in\left[p^{\prime}\right] \times^{+}\left[q^{\prime}\right],(x, y)$ oscillates infinitely often above $(\operatorname{st}(p), \operatorname{st}(q))$. Moreover, if $\left(\mu_{0}, \nu_{0}, \mu_{1}, \nu_{1}, \ldots\right)$ is the type sequence of $(x, y)$, we have $\left\{\mu_{n} \mid n \in\right.$ $\omega\} \subseteq \operatorname{split}\left(p^{\prime}\right)$ and $\left\{\nu_{n} \mid n \in \omega\right\} \subseteq \operatorname{split}\left(q^{\prime}\right)$.

Hence for every $(x, y) \in\left[p^{\prime}\right] \times\left[q^{\prime}\right],\left(p^{\prime}, q^{\prime}\right)$ as in fact 0.0 .2 , there is a unique associated sequence $\left(k_{i}\right)_{i \in \omega}$ in $\omega$, which is determined solely by $(x, y)$ and $(\operatorname{st}(p), \operatorname{st}(q))=:(\sigma, \tau)$. Define the 0 -type pair of the pair of branches $(x, y)$ by $\operatorname{tp}_{\sigma, \tau^{-}} 0$-pair $(x, y):=(\sigma, \tau)$ and for every $n \in \omega$ define $\operatorname{tp}_{\sigma, \tau^{-}}(2 n+1)$-pair $(x, y)=$ $\left(x\left\lceil k_{2 n+1}, y\left\lceil k_{2 n}\right)\right.\right.$ and $\operatorname{tp}_{\sigma, \tau^{-}}(2 n+2)$-pair $(x, y)=\left(x\left\lceil k_{2 n+1}, y\left\lceil k_{2 n+2}\right)\right.\right.$. Using this we can define a partial function

$$
\operatorname{tp}_{\sigma, \tau}^{p^{\prime}, q^{\prime}}:\left({ }^{<\omega} \omega\right)^{2} \rightarrow \omega
$$


by letting $\operatorname{tp}_{\sigma, \tau}^{p^{\prime}, q^{\prime}}(\mu, \nu)=n$ if and only if there exists $(x, y) \in\left[p^{\prime}\right] \times^{+}\left[q^{\prime}\right]$ such

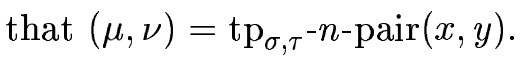

For $(\mu, \nu) \in p^{\prime} \times q^{\prime}$ with $\operatorname{tp}_{\sigma, \tau}^{p^{\prime}, q^{\prime}}(\mu, \nu)=2 n$ there exists a unique sequence $\left(\mu_{0}, \nu_{0}, \ldots, \mu_{n}, \nu_{n}\right)$, which is the initial sequence of length $2 n$ of the type $e_{\sigma, \tau^{-}}$ sequence of $(x, y)$ for some infinitely oscillating $(x, y) \in\left[p^{\prime}\right] \times^{+}\left[q^{\prime}\right]$ over $(\sigma, \tau)$. We call this sequence the type ${ }_{\sigma, \tau^{-}}$sequence of the pair $(\mu, \nu)$ and define $\operatorname{tp}_{\sigma, \tau^{-}}$ $i$-pair $(\mu, \nu)=\operatorname{tp}_{\sigma, \tau^{-}}-$-pair $(x, y)$ for all $i \leq 2 n$. And similar for $(\mu, \nu) \in p^{\prime} \times q^{\prime}$ with $\operatorname{tp}_{\sigma, \tau}^{p^{\prime}, q^{\prime}}(\mu, \nu)$ is odd.

If $(\sigma, \tau)$ or $\left(p^{\prime}, q^{\prime}\right)$ are clear from context, we omit them in the above notation. For $(p, q) \in \mathbb{M}^{2}$ and $(\sigma, \tau) \in p \times q$ let

$$
\operatorname{TP}_{\sigma, \tau}(p, q):=\operatorname{dom}\left(\operatorname{tp}_{\sigma, \tau}^{p, q}\right) \cap\left(\operatorname{split}\left((p)_{\sigma}\right) \times{ }^{+} \operatorname{split}\left((q)_{\tau}\right)\right),
$$

the set of all type pairs of $(p, q)$, and for $n \in \omega$ let $\operatorname{TP}_{\sigma, \tau}^{n}(p, q)$ denote the set of all type pairs of $(p, q)$ of type $n$. For $(\mu, \nu) \in \mathrm{TP}_{\sigma, \tau}^{n}$ with $n$ even let

$$
\begin{aligned}
& \operatorname{Sop}_{\sigma, \tau}^{p, q}(\mu, \nu):=\left\{\mu^{\prime} \in \operatorname{split}\left(p_{\mu}\right) \mid \operatorname{tp}_{\sigma, \tau}^{p, q}\left(\mu^{\prime}, \nu\right)\right.=n+1 \wedge \\
&\left.\operatorname{tp}_{\sigma, \tau}-n-\operatorname{pair}\left(\mu^{\prime}, \nu\right)=(\mu, \nu)\right\},
\end{aligned}
$$

the set of all possible successive oscillation points, and similar, we define $\operatorname{Sop}_{\sigma, \tau}^{p, q}(\mu, \nu)$, if $n$ is odd.

Note that for $\left(p^{\prime}, q^{\prime}\right)$ as in fact 0.0 .2 and $(u, v) \leq\left(p^{\prime}, q^{\prime}\right)$ and $(\sigma, \tau) \in u \times v$ we in general have $\operatorname{TP}_{\sigma, \tau}\left(p^{\prime}, q^{\prime}\right) \cap(u \times v) \neq \mathrm{TP}_{\sigma, \tau}(u, v)$, but there exists $\left(u^{\prime}, v^{\prime}\right) \leq 0$ $(u, v)$ such that for almost all $n \in \omega$ and every $(x, y) \in\left[u^{\prime}\right] \times^{+}\left[v^{\prime}\right]$ we have $\operatorname{tp}_{\sigma, \tau^{-}}$-pair $(x, y) \in \operatorname{TP}_{\sigma, \tau}\left(u^{\prime}, v^{\prime}\right)$ (and therefore $\operatorname{tp}_{\sigma, \tau^{-}}$-pair $(x, y) \in \operatorname{split}\left(u^{\prime}\right) \times$ $\left.\operatorname{split}\left(v^{\prime}\right)\right)$.

For the rest of the paper we always assume that we work with elements $(p, q) \in$ $\mathbb{M}^{2}$, which have the property of $\left(p^{\prime}, q^{\prime}\right)$ of fact 0.0 .2 . By the fact, the set of all such $(p, q)$ is dense in $\mathbb{M}^{2}$, so with this partial ordering we get forcing extensions isomorphic to those of $\mathbb{M}^{2}$.

We will need the following result of Miller, which is not very difficult to prove:

Fact 0.0.3 [Mi] For every colouring of the splitnodes of a Miller tree $p$ in finitely many colours, there exists a $q \in \mathbb{M}$ with $q \leq p$ and such that all splitnodes of $q$ have the same colour.

Ideals: For every ideal $\mathcal{I}$ on ${ }^{\omega} \omega$, its additivity and its covering number is defined by

$$
\begin{aligned}
& \operatorname{add}(\mathcal{I}):=\min \{|F| \mid F \subseteq \mathcal{I} \text { and } \bigcup F \notin \mathcal{I}\} \\
& \operatorname{cov}(\mathcal{I}):=\min \left\{|F| \mid F \subseteq \mathcal{I} \text { and } \bigcup F={ }^{\omega} \omega\right\}
\end{aligned}
$$

Let $\mathcal{M}$ denote the ideal of the meager subsets of ${ }^{\omega} \omega$.

Forcing: For a forcing $P$, an $M$-generic filter $G$ for $P$ and a $P$-name $\dot{a}$ we write 
$\operatorname{val}_{G}(\dot{a})$ or $\dot{a}_{G}$ for the value of $\dot{a}$. For elements $x$ of the ground model we do not distinguish between the canonical name $\check{x}$ and $x$ itself.

Recall that a forcing $P$ has the Laver property if there exists $F \in{ }^{\omega} \omega \cap V$ such that for every $P$-name $\dot{f}$ for an element of ${ }^{\omega} \omega$, every $p \in P$ and every $g \in{ }^{\omega} \omega \cap V$ with $p \Vdash_{P} \forall n \in \omega(\dot{f}(n) \leq g(n))$, there exist $q \leq p$ and $\left(H_{n}\right)_{n \in \omega}$ in $V$ such that $\left|H_{n}\right| \leq F(n)$ and $q \Vdash_{P} \forall n\left(\dot{f}(n) \in H_{n}\right)$. 


\section{Chapter 1}

\section{The consistency of $\operatorname{cov}(\mathcal{M})<\operatorname{add}\left(\mathcal{J}\left(\mathbb{M}^{2}\right)\right)$}

\subsection{An amoeba forcing for $\mathbb{M}^{2}$}

We repeat the definition of the introduction:

\section{Definition 1.1.1 Let}

$$
\begin{aligned}
& \mathrm{J}\left(\mathbb{M}^{n}\right):=\left\{X \subseteq\left({ }^{\omega} \omega\right)^{n} \mid \forall\left(p_{0}, \ldots, p_{n-1}\right) \in \mathbb{M}^{n} \exists\left(q_{0}, \ldots, q_{n-1}\right) \in \mathbb{M}^{n}\right. \\
&\left.\left(\left(q_{0}, \ldots, q_{n-1}\right) \leq\left(p_{0}, \ldots, p_{n-1}\right) \wedge\left(\left[q_{0}\right] \times \ldots \times\left[q_{n-1}\right]\right) \cap X=\emptyset\right)\right\} .
\end{aligned}
$$

It is easy to see that $J(\mathbb{M})$ is a $\sigma$-ideal. Jossen and Spinas [JoSp] have shown that $\mathrm{J}\left(\mathbb{M}^{2}\right)$ is a $\sigma$-ideal but $J\left(\mathbb{M}^{n}\right)$ is not for $n>2$. And they pointed out parallelisms between the behaviour of $\mathcal{J}(\mathbb{M})$ and $\mathcal{J}\left(\mathbb{M}^{2}\right)$. Here, we underpin this by showing that, analogous to the one-dimensional case, we have the following:

Theorem 1.1.2 It is relatively consistent with $Z F C$ that $\operatorname{add}\left(J\left(\mathbb{M}^{2}\right)\right)=\omega_{2}$ and $\operatorname{cov}(\mathcal{M})=\omega_{1}$.

For this define an amoeba forcing $\mathbb{A}\left(\mathbb{M}^{2}\right)$ for $\mathbb{M}^{2}$ as follows:

Definition 1.1.3 Let $\mathbb{A}\left(\mathbb{M}^{2}\right)$ be the set of all pairs $((s, p),(t, q))$ such that

(i) $(p, q) \in \mathbb{M}^{2}, s: n \rightarrow \operatorname{split}(p)$ and $t: m \rightarrow \operatorname{split}(q)$ for some $n, m \in \omega \backslash\{0\}$ with $n=m$ or $n=m+1$;

(ii) the downward closure $S$ of $\operatorname{ran}(s)$ is a finite subtree of $p$ and $\operatorname{split}(S) \subseteq$ $\operatorname{ran}(s)$, the downward closure $T$ of $\operatorname{ran}(t)$ is a finite subtree of $q$ with $\operatorname{split}(T) \subseteq \operatorname{ran}(t)$; 
(iii) the mappings $\Phi:(\operatorname{ran}(s), \subseteq) \rightarrow(\operatorname{ran}(T\lceil n), \subseteq)$ and $\Psi:(\operatorname{ran}(t), \subseteq) \rightarrow$ $(\operatorname{ran}(T\lceil m), \subseteq)$ defined by $s(k) \longmapsto T(k)$ and $t(k) \longmapsto T(k)$ are both isomorphisms, where $T$ is the order preserving enumeration of our wellordering $\prec$ of $<\omega \omega$ fixed in the preliminaries;

(iv) if $s(j)$ is the immediate predecessor in the tree sense of $s(k+1)$ in $\operatorname{split}(p)$ then $s(k+1)(|s(j)|)>\max (\{\# s(i) \mid i \leq k\} \cup\{\# t(i) \mid i \leq k\})$ and $|s(k+1)|>s(k+1)(|s(j)|)$;

if $t(j)$ is the immediate predecessor in the tree sense of $t(k+1)$ in $\operatorname{split}(q)$ then $t(k+1)(|t(j)|)>\max (\{\# s(i) \mid i \leq k+1\} \cup\{\# t(i) \mid i \leq k\})$ and $|t(k+1)|>t(k+1)(|t(k)|)$.

Define a partial ordering $\leq$ on $\mathbb{A}\left(\mathbb{M}^{2}\right)$ by

$$
((s, p),(t, q)) \leq\left(\left(s^{\prime}, p^{\prime}\right),\left(t^{\prime}, q^{\prime}\right)\right): \Leftrightarrow s \supseteq s^{\prime}, t \supseteq t^{\prime}, p \subseteq p^{\prime} \text { and } q \subseteq q^{\prime} .
$$

Oftenly we will write $s$ and $t$ as tupels.

\section{$1.2 \mathbb{A}\left(\mathbb{M}^{2}\right)$ has the pure decision property}

To rule out that $\mathbb{A}\left(\mathbb{M}^{2}\right)$ adds Cohen-reals, we need the following decision property of $\mathbb{A}\left(\mathbb{M}^{2}\right)$ :

Lemma 1.2.1 $\mathbb{A}\left(\mathbb{M}^{2}\right)$ has the pure decision property.

Here, the pure decision property means that for every $\mathbb{A}\left(\mathbb{M}^{2}\right)$-name $\Theta$ and every $((s, p),(t, q)) \in \mathbb{A}\left(\mathbb{M}^{2}\right)$ such that

$$
((s, p),(t, q)) \Vdash_{\mathbb{A}\left(\mathbb{M}^{2}\right)} \Theta \in\{0,1\}
$$

there exists $\left(\left(s^{\prime}, p^{\prime}\right),\left(t^{\prime}, q^{\prime}\right)\right) \in \mathbb{A}\left(\mathbb{M}^{2}\right)$ with $\left(\left(s^{\prime}, p^{\prime}\right),\left(t^{\prime}, q^{\prime}\right)\right) \leq((s, p),(t, q))$ and $\left(\left(s^{\prime}, p^{\prime}\right),\left(t^{\prime}, q^{\prime}\right)\right)$ decides $\Theta$ (this means there exists $j<2$ such that $\left.\left(\left(s^{\prime}, p^{\prime}\right),\left(t^{\prime}, q^{\prime}\right)\right) \Vdash_{{\mathbb{A}\left(\mathbb{M}^{2}\right)}^{2}} \Theta=j\right)$.

Proof of lemma 1.2.1: Let $\Theta$ be an $\mathbb{A}\left(\mathbb{M}^{2}\right)$-name and $((s, p),(t, q)) \in \mathbb{A}\left(\mathbb{M}^{2}\right)$ such that $((s, p),(t, q)) \Vdash_{{\mathrm{A}\left(\mathbb{M}^{2}\right)}^{2}} \Theta \in\{0,1\}$. Our goal is to construct by a fusion a pair $\left(p^{\prime}, q^{\prime}\right)$ of superperfect trees with $\left(p^{\prime}, q^{\prime}\right) \leq^{0}(p, q),\left(\left(s, p^{\prime}\right),\left(t, q^{\prime}\right)\right) \in \mathbb{A}\left(\mathbb{M}^{2}\right)$ and with the following property:

(*) For all $\left(s^{\prime}, t^{\prime}\right) \in<\omega\left(\operatorname{split}\left(p^{\prime}\right)\right) \times<\omega\left(\operatorname{split}\left(q^{\prime}\right)\right)$, if there is some $(u, v) \leq$ $\left(p^{\prime}, q^{\prime}\right)$ with $\left(\left(s^{\prime}, u\right),\left(t^{\prime}, v\right)\right) \in \mathbb{A}\left(\mathbb{M}^{2}\right),\left(\left(s^{\prime}, u\right),\left(t^{\prime}, v\right)\right) \leq\left(\left(s, p^{\prime}\right),\left(t, q^{\prime}\right)\right)$ and $\left(\left(s^{\prime}, u\right),\left(t^{\prime}, v\right)\right)$ decides $\Theta$, then already $\left(\left(s^{\prime}, p^{\prime}\right),\left(t^{\prime}, q^{\prime}\right)\right)$ decides $\Theta$. 
Suppose $|s|=|t|$, so we have to extend $s$ first. The case $|s|=|t|+1$ is very similar and is not written down. We want to construct sequences $\left(\sigma_{n}\right)_{n \in \omega}$, $\left(\tau_{n}\right)_{n \in \omega}$ in $<\omega \omega$ and $\left(\left(u_{n}^{\prime}, v_{n}^{\prime}\right)\right)_{n \in \omega \backslash\{0\}},\left(\left(u_{n}, v_{n}\right)\right)_{n \in \omega}$ in $\mathbb{M}^{2}$ such that, letting $s_{n}:=s \frown\left\langle\sigma_{0}, \ldots, \sigma_{n-1}\right\rangle$ and $t_{n}:=t \frown\left\langle\tau_{0}, \ldots, \tau_{n-1}\right\rangle$, for every $n \in \omega$ the following hold:

(a) $(p, q) \geq\left(u_{0}, v_{0}\right) \geq\left(u_{n+1}^{\prime}, v_{n+1}^{\prime}\right) \geq\left(u_{n+1}, v_{n+1}\right) \geq\left(u_{n+2}^{\prime}, v_{n+2}^{\prime}\right)$;

(b) $\left(\left(s_{n}, u_{n}\right),\left(t_{n}, v_{n}\right)\right) \in \mathbb{A}\left(\mathbb{M}^{2}\right)$ and $\left(\left(s_{n+1}, u_{n+1}^{\prime}\right),\left(t_{n}, v_{n+1}^{\prime}\right)\right) \in \mathbb{A}\left(\mathbb{M}^{2}\right)$;

(c) for every $\left(s^{\prime}, t^{\prime}\right) \in<\omega\left(\operatorname{ran}\left(s_{n+1}\right)\right) \times<\omega\left(\operatorname{ran}\left(t_{n}\right)\right)$ with $s^{\prime}\left(\left|s^{\prime}\right|-1\right)=$ $\sigma_{n}$, if there exists $(u, v) \in \mathbb{M}^{2}$ such that $\left(\left(s^{\prime}, u\right),\left(t^{\prime}, v\right)\right) \in \mathbb{A}\left(\mathbb{M}^{2}\right)$, $\left(\left(s^{\prime}, u\right),\left(t^{\prime}, v\right)\right) \leq\left(\left(s, u_{n+1}^{\prime}\right),\left(t, v_{n+1}^{\prime}\right)\right)$ and $\left(\left(s^{\prime}, u\right),\left(t^{\prime}, v\right)\right)$ decides $\Theta$, then $\left(\left(s^{\prime}, u_{n+1}^{\prime}\right),\left(t^{\prime}, v_{n+1}^{\prime}\right)\right)$ decides $\Theta$ as well;

and for every $\left(s^{\prime}, t^{\prime}\right) \in<\omega\left(\operatorname{ran}\left(s_{n+1}\right)\right) \times<\omega\left(\operatorname{ran}\left(t_{n+1}\right)\right)$ with $t^{\prime}\left(\left|t^{\prime}\right|-\right.$ $1)=\tau_{n}$, if there exists $(u, v) \in \mathbb{M}^{2}$ with $\left(\left(s^{\prime}, u\right),\left(t^{\prime}, v\right)\right) \in \mathbb{A}\left(\mathbb{M}^{2}\right)$, $\left(\left(s^{\prime}, u\right),\left(t^{\prime}, v\right)\right) \leq\left(\left(s, u_{n+1}\right),\left(t, v_{n+1}\right)\right)$ and $\left(\left(s^{\prime}, u\right),\left(t^{\prime}, v\right)\right)$ decides $\Theta$, then $\left(\left(s^{\prime}, u_{n+1}\right),\left(t^{\prime}, v_{n+1}\right)\right)$ decides $\Theta$ as well.

For beginning the construction define $s_{0}:=s, t_{0}:=t, u_{0}:=p$ and $v_{0}:=q$.

Suppose $s_{n}=s^{\frown}\left\langle\sigma_{0}, \ldots, \sigma_{n-1}\right\rangle, t_{n}=t \frown\left\langle\tau_{0}, \ldots, \tau_{n-1}\right\rangle, u_{n}$ and $v_{n}$ are already defined. Fix $\mu \in \operatorname{ran}\left(s_{n}\right)$ maximal such that whenever the element $\left(\left(s_{n} \frown\langle\sigma\rangle, u_{n}\right),\left(t_{n}, v_{n}\right)\right) \in \mathbb{A}\left(\mathbb{M}^{2}\right)$ extends $\left(\left(s_{n}, u_{n}\right),\left(t_{n}, v_{n}\right)\right)$ we have $\mu \varsubsetneqq \sigma$. Choose $\sigma_{n} \in \operatorname{Succ}_{u_{n}}(\mu)$ such that $\left(\left(s_{n} \frown\left\langle\sigma_{n}\right\rangle, u_{n}\right),\left(t_{n}, v_{n}\right)\right) \in \mathbb{A}\left(\mathbb{M}^{2}\right)$ and define $s_{n+1}:=s_{n} \frown\left\langle\sigma_{n}\right\rangle$.

Claim 1: There exists $\left(u_{n+1}^{\prime}, v_{n+1}^{\prime}\right) \in \mathbb{M}^{2}$ such that $\left(u_{n+1}^{\prime}, v_{n+1}^{\prime}\right) \leq\left(u_{n}, v_{n}\right)$, $\left(\left(s_{n+1}, u_{n+1}^{\prime}\right),\left(t_{n}, v_{n+1}^{\prime}\right)\right)$ is an element of $\mathbb{A}\left(\mathbb{M}^{2}\right)$ and the first part of (c) holds.

Proof of claim 1: Let $\left(\left(\bar{s}_{i}, \bar{t}_{i}\right)\right)_{i<N}$ for some $N \in \omega$ enumerate all pairs $\left(s^{\prime}, t^{\prime}\right) \in<^{<\omega}\left(\operatorname{ran}\left(s_{n+1}\right)\right) \times<\omega\left(\operatorname{ran}\left(t_{n}\right)\right)$ with $s^{\prime}\left(\left|s^{\prime}\right|-1\right)=\sigma_{n}$. We want to consider successively all pairs $\left(\bar{s}_{i}, \bar{t}_{i}\right)$ and thin out $\left(u_{n}, v_{n}\right)$ to get $\left(u_{n+1}^{\prime}, v_{n+1}^{\prime}\right)$. Let $p_{0}:=u_{n}$ and $q_{0}:=v_{n}$ and suppose we have already constructed $\left(p_{i}, q_{i}\right) \in \mathbb{M}^{2}$ for some $i<N$.

Consider $\left(\bar{s}_{i}, \bar{t}_{i}\right)$ and suppose there exists a pair $(u, v) \leq\left(p_{i}, q_{i}\right)$ such that $\left(\left(\bar{s}_{i}, u\right),\left(\bar{t}_{i}, v\right)\right) \in \mathbb{A}\left(\mathbb{M}^{2}\right),\left(\left(\bar{s}_{i}, u\right),\left(\bar{t}_{i}, v\right)\right) \leq\left(\left(s, p_{i}\right),\left(t, q_{i}\right)\right)$ and $\left(\left(\bar{s}_{i}, u\right),\left(\bar{t}_{i}, v\right)\right)$ decides $\Theta$ (if there does not exist such a pair $(u, v)$ we let $p_{i+1}:=p_{i}$ and $\left.q_{i+1}:=q_{i}\right)$. Let $S_{i}$ be the downward closure of $\operatorname{ran}\left(\bar{s}_{i}\right)$ in the tree sense and define

$$
\begin{gathered}
r_{p}:=\bigcup\left\{\left(p_{i}\right)_{\sigma} \mid \sigma \in \operatorname{ran}\left(s_{n+1}\right) \backslash S_{i}\right\}, \\
r_{p}^{\prime}:=\bigcup\left\{\left(p_{i}\right)_{\sigma \frown\langle k\rangle} \mid \sigma \in\left(\operatorname{ran}\left(s_{n+1}\right) \backslash \operatorname{ran}\left(\bar{s}_{i}\right)\right) \cap S_{i} \wedge \sigma \frown\langle k\rangle \notin S_{i}\right\}
\end{gathered}
$$


and let

$$
p_{i+1}:=u \cup r_{p} \cup r_{p}^{\prime}
$$

Then $p_{i+1} \in \mathbb{M}$ and note that every $\sigma \in \operatorname{ran}\left(s_{n+1}\right) \backslash \operatorname{ran}\left(\bar{s}_{i}\right)$ remains an infinite splitnode of $p_{i+1}$. Analogous let $T_{i}$ be the downward closure of $\operatorname{ran}\left(\bar{t}_{i}\right)$ and define

$$
\begin{gathered}
r_{q}:=\bigcup\left\{\left(q_{i}\right)_{\tau} \mid \tau \in \operatorname{ran}\left(t_{n+1}\right) \backslash T_{i}\right\}, \\
\left.r_{q}^{\prime}:=\bigcup\left\{\left(q_{i}\right)_{\tau \curlyvee\langle k\rangle} \mid \tau \in\left(\operatorname{ran}\left(t_{n+1}\right) \backslash \operatorname{ran}\left(\bar{t}_{i}\right)\right) \cap T_{i} \wedge \tau \frown\langle k\rangle \notin T_{i}\right)\right\}
\end{gathered}
$$

and let

$$
q_{i+1}:=v \cup r_{q} \cup r_{q}^{\prime} .
$$

Again, every $\tau \in \operatorname{ran}\left(t_{n}\right) \backslash \operatorname{ran}\left(\bar{t}_{i}\right)$ remains an infinite splitnode of $q_{i+1}$. Easily, $\left(\left(s_{n+1}, p_{i+1}\right),\left(t_{n}, q_{i+1}\right)\right) \in \mathbb{A}\left(\mathbb{M}^{2}\right)$ and $\left(\left(s_{n+1}, p_{i+1}\right),\left(t_{n}, q_{i+1}\right)\right) \leq$ $\left(\left(s_{n+1}, p_{i}\right),\left(t_{n}, q_{i}\right)\right)$. And $\left(\left(\bar{s}_{i}, p_{i+1}\right),\left(\bar{t}_{i}, q_{i+1}\right)\right)$ decides $\Theta$ as well, because for $((\bar{s}, \bar{u}),(\bar{t}, \bar{v})) \leq\left(\left(\bar{s}_{i}, p_{i+1}\right),\left(\bar{t}_{i}, q_{i+1}\right)\right)$ our construction and (iv) of the definition of $\mathbb{A}\left(\mathbb{M}^{2}\right)$ guarantees that we have $\bar{s} \backslash \bar{s}_{i} \subseteq u$ and $\bar{t} \backslash \bar{t}_{i} \subseteq v$ (remember that $\left.\bar{s}_{i}\left(\left|\bar{s}_{i}-1\right|\right)=\sigma_{n}\right)$. So for $\overline{\bar{u}}:=\bar{u} \backslash\left(r_{p} \cup r_{p}^{\prime}\right)$ and $\overline{\bar{v}}:=\bar{v} \backslash\left(r_{q} \cup r_{q}^{\prime}\right)$ we have $((\bar{s}, \overline{\bar{u}}),(\bar{t}, \overline{\bar{v}})) \in \mathbb{A}\left(\mathbb{M}^{2}\right)$ and $((\bar{s}, \overline{\bar{u}}),(\bar{t}, \overline{\bar{v}})) \leq\left(\left(\bar{s}_{i}, u\right),\left(\bar{t}_{i}, v\right)\right)$, hence $((\bar{s}, \bar{u}),(\bar{t}, \bar{v}))$ and $\left(\left(\bar{s}_{i}, u\right),\left(\bar{t}_{i}, v\right)\right)$ are compatible, as required.

Finally let $u_{n+1}^{\prime}:=p_{N}$ and $v_{n+1}^{\prime}:=q_{N}$. Then $\left(u_{n+1}^{\prime}, v_{n+1}^{\prime}\right)$ is as desired.

$\square($ claim1)

Fix now $\nu \in \operatorname{ran}\left(t_{n}\right)$, the point where we have to extend the finite tree generated by $\operatorname{ran}\left(t_{n}\right)$. Choose an element $\tau_{n} \in \operatorname{Succ}_{v_{n+1}^{\prime}}(\nu)$ such that we have $\left(\left(s_{n+1}, u_{n+1}^{\prime}\right),\left(t_{n} \frown\langle\tau\rangle\right)\right) \in \mathbb{A}\left(\mathbb{M}^{2}\right)$ and define $t_{n+1}:=t_{n} \frown\left\langle\tau_{n}\right\rangle$. In the same way as we got claim 1 , we get

Claim 2: There exists $\left(u_{n+1}, v_{n+1}\right) \in \mathbb{M}^{2}$ such that $\left(u_{n+1}, v_{n+1}\right) \leq\left(u_{n+1}^{\prime}, v_{n+1}^{\prime}\right)$, $\left(\left(s_{n+1}, u_{n+1}\right),\left(t_{n+1}, v_{n+1}\right)\right) \in \mathbb{A}\left(\mathbb{M}^{2}\right)$ and for every $\left(s^{\prime}, t^{\prime}\right) \in<\omega\left(\operatorname{ran}\left(s_{n+1}\right)\right) \times$ $<\omega\left(\operatorname{ran}\left(t_{n+1}\right)\right)$ with $t^{\prime}\left(\left|t^{\prime}\right|-1\right)=\tau_{n}$, if there exists $(u, v) \in \mathbb{M}^{2}$ such that $\left(\left(s^{\prime}, u\right),\left(t^{\prime}, v\right)\right) \in \mathbb{A}\left(\mathbb{M}^{2}\right),\left(\left(s^{\prime}, u\right),\left(t^{\prime}, v\right)\right) \leq\left(\left(s, u_{n+1}\right),\left(t, v_{n+1}\right)\right)$ and the pair $\left(\left(s^{\prime}, u\right),\left(t^{\prime}, v\right)\right)$ decides $\Theta$, then $\left(\left(s^{\prime}, u_{n+1}\right),\left(t^{\prime}, v_{n+1}\right)\right)$ decides $\Theta$ as well.

$\square($ claim 2)

This finishes our construction.

Let $p^{\prime}$ be the unique superperfect tree determined by

$$
\operatorname{split}\left(p^{\prime}\right)=\bigcup_{n \in \omega} \operatorname{ran}\left(s_{n}\right)
$$

and $q^{\prime}$ the unique superperfect tree determined by

$$
\operatorname{split}\left(q^{\prime}\right)=\bigcup_{n \in \omega} \operatorname{ran}\left(t_{n}\right) .
$$

Claim 3: $\left(p^{\prime}, q^{\prime}\right)$ satisfies property $(*)$ (see at the beginning of this proof). 
Proof of claim 3: Suppose $\left(s^{\prime}, t^{\prime}\right) \in{ }^{<\omega}\left(\operatorname{split}\left(p^{\prime}\right)\right) \times<\omega\left(\operatorname{split}\left(q^{\prime}\right)\right)$ and there exists some $(u, v) \leq\left(p^{\prime}, q^{\prime}\right)$ with $\left(\left(s^{\prime}, u\right),\left(t^{\prime}, v\right)\right) \in \mathbb{A}\left(\mathbb{M}^{2}\right),\left(\left(s^{\prime}, u\right),\left(t^{\prime}, v\right)\right) \leq$ $\left(\left(s, p^{\prime}\right),\left(t, q^{\prime}\right)\right)$ and $\left(\left(s^{\prime}, u\right),\left(t^{\prime}, v\right)\right)$ decides $\Theta$. If $\left|s^{\prime}\right|=\left|t^{\prime}\right|$, there exists $n \in \omega$ with $\left(s^{\prime}, t^{\prime}\right) \in<\omega\left(\operatorname{ran}\left(s_{n+1}\right)\right) \times<\omega\left(\operatorname{ran}\left(t_{n+1}\right)\right)$ and $t^{\prime}\left(\left|t^{\prime}\right|-1\right)=\tau_{n}$. And if $\left|s^{\prime}\right|=$ $\left|t^{\prime}\right|+1$ there exists $n \in \omega$ such that $\left(s^{\prime}, t^{\prime}\right) \in<\omega\left(\operatorname{ran}\left(s_{n+1}\right)\right) \times<\omega\left(\operatorname{ran}\left(t_{n}\right)\right)$ and $s^{\prime}\left(\left|s^{\prime}\right|-1\right)=\sigma_{n}$. Since

$$
\begin{aligned}
\left(\left(s^{\prime}, u\right),\left(t^{\prime}, v\right)\right) \leq & \left(\left(s^{\prime}, p^{\prime}\right),\left(t^{\prime}, q^{\prime}\right)\right) \leq \\
& \left(\left(s^{\prime}, u_{n+1}\right),\left(t^{\prime}, v_{n+1}\right)\right) \leq\left(\left(s^{\prime}, u_{n+1}^{\prime}\right),\left(t^{\prime}, v_{n+1}^{\prime}\right)\right),
\end{aligned}
$$

property (c) gives us that $\left(\left(s^{\prime}, u_{n+1}^{\prime}\right),\left(t^{\prime}, v_{n+1}^{\prime}\right)\right)$ or $\left(\left(s^{\prime}, u_{n+1}\right),\left(t^{\prime}, v_{n+1}\right)\right)$ decides $\Theta$, so $\left(\left(s^{\prime}, p^{\prime}\right),\left(t^{\prime}, q^{\prime}\right)\right)$ decides $\Theta$ as well.

$\square($ claim 3)

Claim 4: $\left(\left(s, p^{\prime}\right),\left(t, q^{\prime}\right)\right)$ decides $\Theta$.

Proof of claim 4: Suppose not. And suppose again that we have $|s|=$ $|t|$; the case $|s|=|t|+1$ is once more similar. We will construct sequences $\left(\left(u_{n}, v_{n}\right)\right)_{n \in \omega},\left(\left(u_{n}^{\prime}, v_{n}^{\prime}\right)\right)_{n \in \omega}$ in $\mathbb{M}^{2}$ and $\left(s_{n}\right)_{n \in \omega}$ and $\left(t_{n}\right)_{n \in \omega}$ in $<\omega(<\omega \omega)$ with $s_{n}=s \frown\left\langle\sigma_{0}, \ldots \sigma_{n-1}\right\rangle$ and $t_{n}=t \frown\left\langle\tau_{0}, \ldots, \tau_{n-1}\right\rangle$ such that for every $n \in \omega$ the following hold:

(a') $s \subseteq s_{n} \subseteq s_{n+1}$ and $t \subseteq t_{n} \subseteq t_{n+1}$;

(b') $\left(p^{\prime}, q^{\prime}\right) \geq\left(u_{n}^{\prime}, v_{n}^{\prime}\right) \geq\left(u_{n}, v_{n}\right) \geq\left(u_{n+1}^{\prime}, v_{n+1}^{\prime}\right)$;

(c') $\left(\left(s_{n+1}, p^{\prime}\right),\left(t_{n+1}, q^{\prime}\right)\right) \in \mathbb{A}\left(\mathbb{M}^{2}\right)$ and $\left(\left(s_{n+1}, p^{\prime}\right),\left(t_{n}, q^{\prime}\right)\right) \in \mathbb{A}\left(\mathbb{M}^{2}\right)$;

(d') if $\left(s^{\prime}, t^{\prime}\right) \in<\omega\left(\operatorname{ran}\left(s_{n}\right)\right) \times<\omega\left(\operatorname{ran}\left(t_{n}\right)\right)$ such that $\left|s^{\prime}\right|=\left|t^{\prime}\right|, t^{\prime}\left(\left|t^{\prime}\right|-1\right)=$ $\tau_{n-1}$ for $n>0,\left(\left(s^{\prime}, u_{n}^{\prime}\right),\left(t^{\prime}, v_{n}^{\prime}\right)\right) \in \mathbb{A}\left(\mathbb{M}^{2}\right)$ and $\left(\left(s^{\prime}, u_{n}^{\prime}\right),\left(t^{\prime}, v_{n}^{\prime}\right)\right) \leq$ $\left(\left(s, u_{n}^{\prime}\right),\left(t, v_{n}^{\prime}\right)\right)$, then there is no $\sigma \in \operatorname{split}\left(u_{n}^{\prime}\right)$ such that we have $\left(\left(s^{\prime} \frown\langle\sigma\rangle, u_{n}^{\prime}\right),\left(t^{\prime}, v_{n}^{\prime}\right)\right) \in \mathbb{A}\left(\mathbb{M}^{2}\right)$ and $\left(\left(s^{\prime} \frown\langle\sigma\rangle, u_{n}^{\prime}\right),\left(t^{\prime}, v_{n}^{\prime}\right)\right)$ decides $\Theta ;$

(e') if $\left(s^{\prime}, t^{\prime}\right) \in<^{<\omega}\left(\operatorname{ran}\left(s_{n+1}\right)\right) \times<\omega\left(\operatorname{ran}\left(t_{n}\right)\right)$ such that $\left|s^{\prime}\right|=\left|t^{\prime}\right|+1, s^{\prime}\left(\left|s^{\prime}\right|-1\right)=$ $\sigma_{n},\left(\left(s^{\prime}, u_{n}\right),\left(t^{\prime}, v_{n}\right)\right) \in \mathbb{A}\left(\mathbb{M}^{2}\right)$ and $\left(\left(s^{\prime}, u_{n}\right),\left(t^{\prime}, v_{n}\right)\right) \leq\left(\left(s, u_{n}\right),\left(t, v_{n}\right)\right)$, then there is no $\tau \in \operatorname{split}\left(v_{n}\right)$ such that we have $\left(\left(s^{\prime}, u_{n}\right),\left(t^{\prime}\left\langle\langle\tau\rangle, v_{n}\right)\right) \in\right.$ $\mathbb{A}\left(\mathbb{M}^{2}\right)$ and $\left(\left(s^{\prime}, u_{n}\right),\left(t^{\prime} \frown\langle\tau\rangle, v_{n}\right)\right)$ decides $\Theta$.

Define $s:=s_{0}$ and $t:=t_{0}$. For beginning the induction fix $\mu \in \operatorname{ran}(s)$, the unique point where we can extend the finite subtree of $p$ generated by $\operatorname{ran}(s)$, and prune $p^{\prime}$ by defining

$$
\bar{p}:=\bigcup\left\{\left(p^{\prime}\right)_{\mu \smile\langle k\rangle} \mid \forall \sigma \in \operatorname{ran}(s) \backslash\{\mu\}(\mu \frown\langle k\rangle \nsubseteq \sigma)\right\} .
$$

Then $\bar{p}$ is a superperfect tree with stem $\mu$ and for $\rho \in \operatorname{Succ}_{\bar{p}}(\mu)$ we have $(\bar{p})_{\rho}=$ $\left(p^{\prime}\right)_{\rho}$. For every $\rho \in \operatorname{Succ}_{\bar{p}}(\mu)$ we want to colour the set

$$
S_{\rho}:=\left\{\sigma \in \operatorname{split}\left(p^{\prime}\right)_{\rho} \mid\left(\left(s \frown\langle\sigma\rangle, p^{\prime}\right),\left(t, q^{\prime}\right)\right) \in \mathbb{A}\left(\mathbb{M}^{2}\right)\right\}
$$


in three colours by defining

$$
\begin{aligned}
\varphi_{\rho}: S_{\rho} & \longrightarrow\{0,1,2\} \\
\sigma & \longmapsto \begin{cases}0, & \text { if }\left(\left(s^{\curlyvee}\langle\sigma\rangle, p^{\prime}\right),\left(t, q^{\prime}\right)\right) \Vdash_{\left.\mathbb{A}_{(\mathbb{M}}^{2}\right)} \Theta=0, \\
1, & \text { if }\left(\left(s \smile\langle\sigma\rangle, p^{\prime}\right),\left(t, q^{\prime}\right)\right) \Vdash_{\left.\mathbb{A}_{(\mathbb{M}}^{2}\right)} \Theta=1, \\
2, & \text { if }\left(\left(s^{\curlyvee}\langle\sigma\rangle, p^{\prime}\right),\left(t, q^{\prime}\right)\right) \text { does not decide } \Theta .\end{cases}
\end{aligned}
$$

Fact 0.0 .3 gives us for every $\rho \in \operatorname{Succ}_{\bar{p}}(\mu)$ a superperfect tree $p^{\rho} \subseteq\left(p^{\prime}\right)_{\rho}$ whose splitnodes all have colour $\varepsilon_{\rho}$.

If there is an infinite set $A \subseteq \operatorname{Succ}_{\bar{p}}(\mu)$ and an $\varepsilon \in\{0,1\}$ such that for every $\rho \in A$ we have $\varepsilon_{\rho}=\varepsilon$, then we can define an element of $\mathbb{A}\left(\mathbb{M}^{2}\right)$ which forces $\Theta$ to be this $\varepsilon$ as follows: Define

$$
\bar{p}^{\prime}:=\bigcup\left\{p^{\rho} \mid \rho \in A\right\} \cup\left(p^{\prime} \backslash \bar{p}\right)
$$

then clearly $\left(\left(s, \bar{p}^{\prime}\right),\left(t, q^{\prime}\right)\right) \Vdash_{{\mathrm{A}\left(\mathbb{M}^{2}\right)}} \Theta=\varepsilon$.

By the property $(*)$ of our constructed pair $\left(p^{\prime}, q^{\prime}\right)$ this implies that $\left(\left(s, p^{\prime}\right),\left(t, q^{\prime}\right)\right)$ decides $\Theta$, a contradiction to our assumption.

So there must exist a finite set $F \subseteq \operatorname{Succ}_{\bar{p}}(\mu)$ such that for every $\rho \in \operatorname{Succ}_{\bar{p}}(\mu) \backslash F$ we have $\varepsilon_{\rho}=2$. Define

$$
u_{0}^{\prime}:=\bigcup\left\{p^{\rho} \mid \rho \in \operatorname{Succ}_{\bar{p}}(\mu) \backslash F\right\} \cup\left(p^{\prime} \backslash \bar{p}\right)
$$

and $v_{0}^{\prime}:=q^{\prime}$. Then for no $\sigma \in \operatorname{split}\left(u_{0}^{\prime}\right)$ with $\left(\left(s \frown\langle\sigma\rangle, u_{0}^{\prime}\right),\left(t, v_{0}^{\prime}\right)\right) \in \mathbb{A}\left(\mathbb{M}^{2}\right)$ the element $\left(\left(s \frown\langle\sigma\rangle, u_{0}^{\prime}\right),\left(t, v_{0}^{\prime}\right)\right)$ decides $\Theta$. Choose $\sigma_{0} \in \operatorname{Succ}_{u_{0}^{\prime}}(\mu)$ such that $\left(\left(s \frown\left\langle\sigma_{0}\right\rangle, u_{0}^{\prime}\right),\left(t, v_{0}^{\prime}\right)\right) \in \mathbb{A}\left(\mathbb{M}^{2}\right)$ and define $s_{1}:=s \frown\left\langle\sigma_{0}\right\rangle$.

Now fix $\nu \in \operatorname{ran}(t)$, the unique point where we can extend the finite subtree generated by $\operatorname{ran}(t)$. We want to do the same as before at the right side: Prune $v_{0}^{\prime}$ by defining

$$
\bar{v}:=\bigcup\left\{\left(v_{0}^{\prime}\right)_{\nu \smile\langle k\rangle} \mid \forall \tau \in \operatorname{ran}(t) \backslash\{\nu\}(\nu \frown\langle k\rangle \nsubseteq \tau)\right\}
$$

and colour for every $\rho \in \operatorname{Succ}_{\bar{v}}(\nu)$ the set

$$
T_{\rho}:=\left\{\tau \in \operatorname{split}\left(v_{0}^{\prime}\right)_{\rho} \mid\left(\left(s_{1}, u_{0}^{\prime}\right),\left(t \frown\langle\tau\rangle, v_{0}^{\prime}\right)\right) \in \mathbb{A}\left(\mathbb{M}^{2}\right)\right\}
$$

by defining

$$
\begin{aligned}
\psi_{\rho}: T_{\rho} & \longrightarrow\{0,1,2\} \\
\tau & \longmapsto \begin{cases}0, & \text { if }\left(\left(s_{1}, u_{0}^{\prime}\right),\left(t^{\frown}\langle\tau\rangle, v_{0}^{\prime}\right)\right) \Vdash_{\mathbb{A}\left(\mathbb{M}^{2}\right)} \Theta=0, \\
1, & \text { if }\left(\left(s_{1}, u_{0}^{\prime}\right),\left(t^{\frown}\langle\tau\rangle, v_{0}^{\prime}\right)\right) \Vdash_{\mathbb{A}\left(\mathbb{M}^{2}\right)} \Theta=1, \\
2, & \text { if }\left(\left(s_{1}, u_{0}^{\prime}\right),\left(t^{\frown}\langle\tau\rangle, v_{0}^{\prime}\right)\right) \text { does not decide } \Theta .\end{cases}
\end{aligned}
$$

Again we get for every $\rho \in \operatorname{Succ}_{\bar{v}}(\nu)$ a superperfect tree $q^{\rho} \subseteq\left(v_{0}^{\prime}\right)_{\rho}$, whose splitnodes all have colour $\varepsilon_{\rho}$. If there exists an infinite set $A \subseteq \operatorname{Succ}_{\bar{v}}(\nu)$ and $\varepsilon \in\{0,1\}$, such that for every $\rho \in A$ we have $\varepsilon_{\rho}=\varepsilon$, we can define 
$\bar{v}^{\prime}$ such that $\left(\left(s_{1}, u_{0}^{\prime}\right),\left(t, \bar{v}^{\prime}\right)\right) \Vdash_{\left.\mathbb{A}_{(\mathbb{M}}^{2}\right)} \Theta=\varepsilon$ and therefore - again by property $(*)-\left(\left(s_{1}, p^{\prime}\right),\left(t, q^{\prime}\right)\right)$ decides $\Theta$. But $\left(\left(s_{1}, u_{0}^{\prime}\right),\left(t, v_{0}^{\prime}\right)\right) \leq\left(\left(s_{1}, p^{\prime}\right),\left(t, q^{\prime}\right)\right)$, hence $\left(\left(s_{1}, u_{0}^{\prime}\right),\left(t, v_{0}^{\prime}\right)\right)$ decides $\Theta$, this is a contradiction to the choice of $\left(u_{0}^{\prime}, v_{0}^{\prime}\right)$ (property (d') for $n=0$ ). Hence we get a finite set $F \subseteq \operatorname{Succ}_{\bar{v}}(\nu)$ with $\varepsilon_{\rho}=2$ for every $\rho \in \operatorname{Succ}_{\bar{v}}(\nu) \backslash F$. Define

$$
v_{0}:=\bigcup\left\{q^{\rho} \mid \rho \in \operatorname{Succ}_{\bar{v}}(\nu) \backslash F\right\} \cup\left(v_{0}^{\prime} \backslash \bar{v}\right)
$$

and $u_{0}:=u_{0}^{\prime}$. Then for no $\tau \in \operatorname{split}\left(v_{0}\right)$ with $\left(\left(s_{1}, u_{0}\right),\left(t^{\frown}\langle\tau\rangle, v_{0}\right)\right) \in \mathbb{A}\left(\mathbb{M}^{2}\right)$ the element $\left(\left(s_{1}, u_{0}\right),\left(t \frown\langle\tau\rangle, v_{0}\right)\right)$ decides $\Theta$. Choose $\tau_{0} \in \operatorname{Succ}_{v_{0}}(\nu)$ with $\left(\left(s_{1}, u_{0}\right),\left(\tau \frown\left\langle\tau_{0}\right\rangle, v_{0}\right)\right) \in \mathbb{A}\left(\mathbb{M}^{2}\right)$ and define $t_{1}:=t \frown\left\langle\tau_{0}\right\rangle$.

Suppose we have constructed $\left(\left(u_{i}, v_{i}\right)\right)_{i \leq n},\left(\left(u_{i}^{\prime}, v_{i}^{\prime}\right)\right)_{i \leq n},\left(s_{i}\right)_{i \leq n+1}$ and $\left(t_{i}\right)_{i \leq n+1}$ with $s_{i}=s \frown\left\langle\sigma_{0}, \ldots \sigma_{i-1}\right\rangle$ and $t_{i}=t \frown\left\langle\tau_{0}, \ldots, \tau_{i-1}\right\rangle$ for some $n \in \omega$. First we will describe how to get $\left(u_{n+1}^{\prime}, v_{n+1}^{\prime}\right)$ : Let $\left(\left(\bar{s}_{i}, \bar{t}_{i}\right)_{i<N}\right.$ be an enumeration of all $\left(s^{\prime}, t^{\prime}\right) \in<\omega\left(\operatorname{ran}\left(s_{n+1}\right)\right) \times<\omega\left(\operatorname{ran}\left(t_{n+1}\right)\right)$ with $\left|s^{\prime}\right|=\left|t^{\prime}\right|$ and $t^{\prime}\left(\left|t^{\prime}\right|-1\right)=\tau_{n}$. By considering successively all pairs $\left(\bar{s}_{i}, \bar{t}_{i}\right)$, we will thin out $\left(u_{n}, v_{n}\right)$ step by step to make (d') true. Let $p_{0}:=u_{n}$ and $q_{0}:=v_{n}$. Suppose $\left(p_{i}, q_{i}\right)$ is already constructed for some $i<N$. Consider $\left(\bar{s}_{i}, \bar{t}_{i}\right)$ and fix $\mu \in \operatorname{ran}\left(\bar{s}_{i}\right)$, the unique point where the finite subtree of $u_{n}$ generated by $\bar{s}_{i}$ can be extended. Prune $p_{i}$ by defining

$$
\bar{p}_{i}:=\bigcup\left\{\left(p_{i}\right)_{\mu \smile\langle k\rangle} \mid \forall \sigma \in \operatorname{ran}\left(s_{n}\right) \backslash\{\mu\}(\mu \smile\langle k\rangle \nsubseteq \sigma)\right\} .
$$

For every $\rho \in \operatorname{Succ}_{\bar{p}}(\mu)$ we colour the set

$$
S_{\rho}^{i}:=\left\{\sigma \in \operatorname{split}\left(p_{i}\right)_{\rho} \mid\left(\left(\bar{s}_{i} \frown\langle\sigma\rangle, p_{i}\right),\left(\bar{t}_{i}, q_{i}\right)\right) \in \mathbb{A}\left(\mathbb{M}^{2}\right)\right\}
$$

with the three colours 0,1 and 2 as in the first step of the induction. We get superperfect trees $p^{\rho} \subseteq\left(p_{i}\right)_{\rho}$ whose splitnodes all have colour $\varepsilon_{\rho}$.

If there is an $\varepsilon \in\{0,1\}$ such that for infinitely many $\rho \in \operatorname{Succ}_{\bar{p}_{i}}(\mu)$ we have $\varepsilon_{\rho}=\varepsilon$, we can easily define $(\bar{u}, \bar{v})$ such that $\left(\left(\bar{s}_{i}, \bar{u}\right),\left(\bar{t}_{i}, \bar{v}\right)\right) \Vdash_{{\mathrm{A}\left(\mathbb{M}^{2}\right)}^{2}} \Theta=\varepsilon$.

By property $(*)$ we have that $\left(\left(\bar{s}_{i}, p^{\prime}\right),\left(\bar{t}_{i}, q^{\prime}\right)\right)$ decides $\Theta$. Fix $k \leq n$ such that $\bar{s}_{i}\left(\left|\bar{s}_{i}\right|-1\right)=\sigma_{k}$, then $\left(\left(\bar{s}_{i}, u_{k}\right),\left(\bar{t}_{i}, v_{k}\right)\right) \leq\left(\left(\bar{s}_{i}, p^{\prime}\right),\left(\bar{t}_{i}, q^{\prime}\right)\right)$ and therefore $\left(\left(\bar{s}_{i}, u_{k}\right),\left(\bar{t}_{i}, v_{k}\right)\right)$ decides $\Theta$ as well. But $\left(\bar{s}_{i}, \bar{t}_{i}\left\lceil\left(\left|\bar{t}_{i}\right|-1\right)\right) \in<\omega\left(\operatorname{ran}\left(s_{k+1}\right)\right) \times\right.$ $<\omega\left(\operatorname{ran}\left(t_{k}\right)\right)$ and $\left.\left|\bar{s}_{i}\right|=\mid \bar{t}_{i} \uparrow\left(\left|\bar{t}_{i}\right|-1\right)\right) \mid+1$, hence the element $\left(\left(\bar{s}_{i}, u_{k}\right),\left(\bar{t}_{i}, v_{k}\right)\right)$ does not decide $\Theta$ by property $\left(e^{\prime}\right)$ for $k$, a contradiction.

So for all but finitely many $\rho \in \operatorname{Succ}_{p_{i}}(\mu)$ we have $\varepsilon_{\rho}=2$. Define

$$
p_{i+1}:=\bigcup\left\{p^{\rho} \mid \rho \in \operatorname{Succ}_{p_{i}}(\mu) \wedge \varepsilon_{\rho}=2\right\} \cup\left(p_{i} \backslash \bar{p}_{i}\right)
$$

and $q_{i+1}:=q_{i}$.

In the end, we define $u_{n+1}^{\prime}:=p_{N}$ and $v_{n+1}^{\prime}:=q_{N}$. Then $\left(u_{n+1}^{\prime}, v_{n+1}^{\prime}\right)$ is as desired. Choose an element $\sigma_{n+1} \in \operatorname{split}\left(u_{n+1}^{\prime}\right)$ such that we have $\left(\left(s_{n+1} \frown\left\langle\sigma_{0}\right\rangle, u_{n+1}^{\prime}\right),\left(t_{n+1}, v_{n+1}^{\prime}\right)\right) \in \mathbb{A}\left(\mathbb{M}^{2}\right)$ and let $s_{n+2}:=s_{n+1} \frown\left\langle\sigma_{n+1}\right\rangle$. 
Analogously we get $\left(u_{n+1}, v_{n+1}\right) \leq\left(u_{n+1}^{\prime}, v_{n+1}^{\prime}\right)$ in $\mathbb{M}^{2}$ such that property $\left(e^{\prime}\right)$ holds. Choose an element $\tau_{n+1} \in \operatorname{split}\left(v_{n+1}\right)$ such that we have $\left(\left(s_{n+2}, u_{n+1}\right),\left(t_{n+1} \frown\left\langle\tau_{n+1}\right\rangle, v_{n+1}\right)\right) \in \mathbb{A}\left(\mathbb{M}^{2}\right)$ and let $t_{n+2}:=t_{n+1} \frown\left\langle\tau_{n+1}\right\rangle$.

This finishes the construction. Now we can define $u$ and $v$ to be the unique superperfect trees with

$$
\operatorname{split}(u)=\bigcup\left\{\operatorname{ran}\left(s_{n}\right) \mid n \in \omega\right\}
$$

and

$$
\operatorname{split}(v)=\bigcup\left\{\operatorname{ran}\left(t_{n}\right) \mid n \in \omega\right\} .
$$

Then $((s, u),(t, v)) \in \mathbb{A}\left(\mathbb{M}^{2}\right)$ and the construction and property $(*)$ guarantee that no extension of $((s, u),(t, v))$ decides $\Theta$, which is a contradiction. So claim 4 is proven and we are done.

$\square($ claim 4)

Corollary 1.2.2 $\mathbb{A}\left(\mathbb{M}^{2}\right)$ has the following decision property:

If $\Theta$ is an $\mathbb{A}\left(\mathbb{M}^{2}\right)$-name and $((s, p),(t, q)) \in \mathbb{A}\left(\mathbb{M}^{2}\right)$ such that for some $n \in \omega$

$$
((s, p),(t, q)) \Vdash_{\mathbb{A}\left(\mathbb{M}^{2}\right)} \Theta<n,
$$

then there exists an element $\left(\left(s, p^{\prime}\right),\left(t, q^{\prime}\right)\right) \in \mathbb{A}\left(\mathbb{M}^{2}\right)$ with $\left(\left(s, p^{\prime}\right),\left(t, q^{\prime}\right)\right) \leq$ $((s, p),(t, q))$ and $\left(\left(s, p^{\prime}\right),\left(t, q^{\prime}\right)\right)$ decides $\Theta$.

Proof: Suppose $((s, t),(t, q)) \Vdash_{{\mathbb{A}\left(\mathbb{M}^{2}\right)}^{2}} \Theta<n$. Choose an $\mathbb{A}\left(\mathbb{M}^{2}\right)$-name $\dot{a}_{0}$ such that for every $\mathbb{A}\left(\mathbb{M}^{2}\right)$-generic filter $G$ we have $\left(\dot{a}_{0}\right)_{G}=0$ if and only if $\Theta_{G}=$ 0 and $\left(\dot{a}_{0}\right)_{G}=1$ if and only if $\Theta_{G} \neq 0$. As $\Vdash_{\left.\mathbb{A M}^{2}\right)} \dot{a}_{0} \in\{0,1\}$, therefore $((s, p),(t, q)) \Vdash_{{\mathbb{A}\left(\mathbb{M}^{2}\right)}} \dot{a}_{0} \in\{0,1\}$, the pure decision property of $\mathbb{A}\left(\mathbb{M}^{2}\right)$ gives us a pair $\left(p_{0}, q_{0}\right) \in \mathbb{M}^{2}$ such that $\left(p_{0}, q_{0}\right) \leq(p, q),\left(\left(s, p_{0}\right),\left(t, q_{0}\right)\right) \in \mathbb{A}\left(\mathbb{M}^{2}\right)$ and $\left(\left(s, p_{0}\right),\left(t, q_{0}\right)\right)$ decides $\dot{a}_{0}$. If $\left(\left(s, p_{0}\right),\left(t, q_{0}\right)\right) \Vdash_{\left.\mathbb{A}_{(\mathbb{M}}^{2}\right)} \dot{a}_{0}=0$ define $p^{\prime}:=p_{0}$ and $q^{\prime}:=q_{0}$; if $\left(\left(s, p_{0}\right),\left(t, q_{0}\right)\right) \Vdash_{\left.\mathbb{A}_{(\mathbb{M}}^{2}\right)} \dot{a}_{0}=1$ choose an $\mathbb{A}\left(\mathbb{M}^{2}\right)$-name $\dot{a}_{1}$ such that for every $\mathbb{A}\left(\mathbb{M}^{2}\right)$-generic filter $G$ we have $\left(\dot{a}_{1}\right)_{G}=0$ if and only if $\Theta_{G}=$ 1 and $\left(\dot{a}_{1}\right)_{G}=1$ if and only if $\Theta_{G} \neq 1$. Again the pure decision property of $\mathbb{A}\left(\mathbb{M}^{2}\right)$ gives us $\left(p_{1}, q_{1}\right) \leq\left(p_{0}, q_{0}\right)$ such that $\left(\left(s, p_{1}\right),\left(t, q_{1}\right)\right)$ decides $\dot{a}_{1}$. If $\left(\left(s, p_{1}\right),\left(t, q_{1}\right)\right) \Vdash_{{\mathrm{A}\left(\mathbb{M}^{2}\right)}^{2}} \dot{a}_{1}=0$ let $p^{\prime}:=p_{1}$ and $q^{\prime}:=q_{1}$; if $\left(\left(s, p_{1}\right),\left(t, q_{1}\right)\right) \Vdash_{\left.\mathrm{A}_{(\mathbb{M}}^{2}\right)}$ $\dot{a}_{0}=1$ choose an $\mathbb{A}\left(\mathbb{M}^{2}\right)$-name $\dot{a}_{2}$ with $\left(\dot{a}_{2}\right)_{G}=0$ if and only if $\Theta_{G}=2$ and $\left(\dot{a}_{2}\right)_{G}=1$ if and only if $\Theta_{G} \neq 2$ and so on. Since $((s, p),(t, q)) \Vdash_{\mathbb{A}_{\left(\mathbb{M}^{2}\right)}} \Theta<n$, this procedure stops after finitely many steps and we get $\left(p^{\prime}, q^{\prime}\right)$ as desired.

\section{3 $\mathbb{A}\left(\mathbb{M}^{2}\right)$ does not add Cohen-reals}

Lemma 1.3.1 $\mathbb{A}\left(\mathbb{M}^{2}\right)$ has the Laver property. 
As an abbreviation we will use the following:

Definition 1.3.2 For $((s, p),(t, q)) \in \mathbb{A}\left(\mathbb{M}^{2}\right)$ and an element $s^{\prime} \in<\omega(\operatorname{ran}(s))$ with $\left(\left(s^{\prime}, p\right),\left(t^{\prime}, q\right)\right) \in \mathbb{A}\left(\mathbb{M}^{2}\right)$ for some $t^{\prime}$ define

$$
p\left(s, s^{\prime}\right):=\bigcup\left\{(p)_{\sigma \smile\langle k\rangle} \mid \sigma \in \operatorname{ran}\left(s^{\prime}\right) \wedge \forall \sigma^{\prime} \in \operatorname{ran}(s) \backslash \operatorname{ran}\left(s^{\prime}\right)\left(\sigma^{\frown}\langle k\rangle \nsubseteq \sigma^{\prime}\right)\right\}
$$

and analogous define a superperfect tree $q\left(t, t^{\prime}\right)$ for $((s, p),(t, q)) \in \mathbb{A}\left(\mathbb{M}^{2}\right)$ and $t^{\prime} \in<\omega(\operatorname{ran}(t))$ with $\left(\left(s^{\prime}, p\right),\left(t^{\prime}, q\right)\right) \in \mathbb{A}\left(\mathbb{M}^{2}\right)$ for some $s^{\prime}$.

Proof of lemma 1.3.1: Suppose $((s, p),(t, q)) \in \mathbb{A}\left(\mathbb{M}^{2}\right), g \in{ }^{\omega} \omega \cap V$ and $\dot{f}$ is an $\mathbb{A}\left(\mathbb{M}^{2}\right)$-name for an element of ${ }^{\omega} \omega$ such that

$$
((s, p),(t, q)) \Vdash_{\mathbb{A}_{\left(\mathbb{M}^{2}\right)}} \forall n(\dot{f}(n)<g(n)) .
$$

We want to construct by a fusion $\left(p^{\prime}, q^{\prime}\right) \in \mathbb{A}\left(\mathbb{M}^{2}\right)$ and a sequence $\left(H_{n}\right)_{n \in \omega}$ in $V$ such that $\left(\left(s, p^{\prime}\right),\left(t, q^{\prime}\right)\right) \in \mathbb{A}\left(\mathbb{M}^{2}\right),\left(\left(s, p^{\prime}\right),\left(t, q^{\prime}\right)\right) \leq((s, p),(t, q)),\left|H_{n}\right| \leq 2^{m_{n}}$ for a strictly increasing sequence $\left(m_{n}\right)_{n \in \omega}$ of natural numbers not depending on $\left(\left(s, p^{\prime}\right),\left(t, q^{\prime}\right)\right), g$ and $\dot{f}$ and $\left(\left(s, p^{\prime}\right),\left(t, q^{\prime}\right)\right) \Vdash_{{\mathrm{A}\left(\mathbb{M}^{2}\right)}^{2}} \forall n\left(\dot{f}(n) \in H_{n}\right)$. Oftenly $m_{n}$ equals $n$, here we have to choose it a little bit larger, but as the specific value of $m_{n}$ is insignificant, we avoid the finitary combinatorics.

Suppose $|s|=|t|$; the case $|s|=|t|+1$ is similar. We construct sequences $\left(s_{n}\right)_{n \in \omega},\left(t_{n}\right)_{n \in \omega}$ in $<\omega(<\omega \omega),\left(\left(u_{n}, v_{n}\right)\right)_{n \in \omega}$ in $\mathbb{M}^{2},\left(m_{n}\right)_{n \in \omega}$ in $\omega$ and a sequence $\left(H_{n}\right)_{n \in \omega}$ of subsets of $\omega$ in $V$ by induction such that for every $n \in \omega$ the following hold:

(i) $(p, q) \geq\left(u_{n}, v_{n}\right) \geq\left(u_{n+1}, v_{n+1}\right)$;

(ii) $\left(\left(s_{n}, u_{n}\right),\left(t_{n}, v_{n}\right)\right) \in \mathbb{A}\left(\mathbb{M}^{2}\right)$;

(iii) $s \subseteq s_{n} \subseteq s_{n+1}$ and $t \subseteq t_{n} \subseteq t_{n+1}$; and if $n$ is even we have $s_{n+1}=s_{n}$ and if $n$ is odd we have $t_{n+1}=t_{n}$;

(iv) $\left(\left(s, u_{n}\right),\left(t, v_{n}\right)\right) \Vdash_{\mathbb{A}\left(\mathbb{M}^{2}\right)} \dot{f}(n) \in H_{n}$;

(v) the sequence $\left(m_{n}\right)_{n \in \omega}$ is strictly increasing and only depends on $n$ and is independent of $\left(\left(s, p^{\prime}\right),\left(t, q^{\prime}\right)\right), g$ and $\dot{f}$ and $\left|H_{n}\right| \leq 2^{m_{n}}$.

For beginning the induction we find a natural number $K$ and $(u, v) \in \mathbb{M}^{2}$ with $((s, u),(t, v)) \leq((s, p),(t, q))$ and $((s, u),(t, v)) \Vdash_{{\mathbb{A}\left(\mathbb{M}^{2}\right)}} \dot{f}(0)=K$ by corollary 1.2.2. Define $\left(u_{0}, v_{0}\right):=(u, v), s_{0}:=s, t_{0}:=t, m_{0}:=0$ and $H_{0}:=\{K\}$.

Suppose we have already constructed $\left(\left(s_{i}, u_{i}\right),\left(t_{i}, v_{i}\right)\right) \in \mathbb{A}\left(\mathbb{M}^{2}\right), m_{i}$ and $H_{i}$ for $i \leq n$ for some $n \in \omega$ and suppose first that $n$ is odd. Fix $\mu \in \operatorname{ran}\left(s_{n}\right)$, the node where we have to extend the finite tree generated by $\operatorname{ran}\left(s_{n}\right)$. Choose $\sigma \in \operatorname{Succ}_{u_{n}}(\mu)$ such that $\left(\left(s_{n} \frown\langle\sigma\rangle, u_{n}\right),\left(t_{n}, v_{n}\right)\right) \in \mathbb{A}\left(\mathbb{M}^{2}\right)$ and define $s_{n+1}:=$ 
$s_{n} \frown\langle\sigma\rangle$ and $t_{n+1}:=t_{n}$.

Claim 1: There exists a pair $(u, v) \in \mathbb{M}^{2}$ such that $(u, v) \leq\left(u_{n}, v_{n}\right)$ and $\left(\left(s_{n+1}, u\right),\left(t_{n+1}, v\right)\right) \in \mathbb{A}\left(\mathbb{M}^{2}\right)$ and for every pair $\left(s^{\prime}, t^{\prime}\right) \in<\omega\left(\operatorname{ran}\left(s_{n+1}\right)\right) \times$ $<\omega\left(\operatorname{ran}\left(t_{n+1}\right)\right)$ with $\left(\left(s^{\prime}, u\right),\left(t^{\prime}, v\right)\right) \in \mathbb{A}\left(\mathbb{M}^{2}\right)$ and $\left(\left(s^{\prime}, u\right),\left(t^{\prime}, v\right)\right) \leq\left(\left(s, u_{n}\right),\left(t, v_{n}\right)\right)$ there exists some $K$ such that

$$
\left(\left(s^{\prime}, u\left(s_{n+1}, s^{\prime}\right)\right),\left(t^{\prime}, v\left(t_{n+1}, t^{\prime}\right)\right)\right) \Vdash_{\mathbb{A}\left(\mathbb{M}^{2}\right)} \dot{f}(n+1)=K .
$$

Proof of claim 1: Let $\left(\left(\bar{s}_{i}, \bar{t}_{i}\right)\right)_{i<N}$ for some $N \in \omega$ enumerate all pairs $\left(s^{\prime}, t^{\prime}\right)$ as in claim 1 . We want to consider successively all pairs $\left(\bar{s}_{i}, \bar{t}_{i}\right)$ and thin out $\left(u_{n}, v_{n}\right)$ step by step to get $(u, v)$ : Define $p_{0}:=u_{n}$ and $q_{0}:=v_{n}$ and suppose we have constructed $\left(p_{i}, q_{i}\right)$ for some $i<N$. Consider $\left(\bar{s}_{i}, \bar{t}_{i}\right)$. Suppose there exists $(\bar{u}, \bar{v}) \in$ $\mathbb{M}^{2}$ with $\left(\left(\bar{s}_{i}, \bar{u}\right),\left(\bar{t}_{i}, \bar{v}\right)\right) \leq\left(\left(s, p_{i}\right),\left(t, q_{i}\right)\right)$ (if not, we let $p_{i+1}:=p_{i}$ and $q_{i+1}:=$ $\left.q_{i}\right)$. It is $\left(\left(s, p_{i}\right),\left(t, q_{i}\right)\right) \leq((s, p),(t, q))$, hence $\left(\left(\bar{s}_{i}, \bar{u}\right),\left(\bar{t}_{i}, \bar{v}\right)\right) \Vdash_{\mathrm{A}_{\left(\mathbb{M}^{2}\right)}} \dot{f}(n+1)<$ $g(n+1)$, and the pure decision property of $\mathbb{A}\left(\mathbb{M}^{2}\right)$, corollary 1.2 .2 to be precise, gives us a pair $(\bar{p}, \bar{q}) \in \mathbb{M}^{2}$ with $\left(\left(\bar{s}_{i}, \bar{p}\right),\left(\bar{t}_{i}, \bar{q}\right)\right) \in \mathbb{A}\left(\mathbb{M}^{2}\right),\left(\left(\bar{s}_{i}, \bar{p}\right),\left(\bar{t}_{i}, \bar{q}\right)\right) \leq$ $\left(\left(\bar{s}_{i}, \bar{u}\right),\left(\bar{t}_{i}, \bar{v}\right)\right)$ and $\left(\left(\bar{s}_{i}, \bar{p}\right),\left(\bar{t}_{i}, \bar{q}\right)\right) \Vdash_{\left.\mathrm{A}_{(\mathbb{M}}^{2}\right)} \dot{f}(n+1)=K$ for some $K$. Clearly, we can thin out $\bar{p}$ and $\bar{q}$ to $\overline{\bar{p}}$ and $\overline{\bar{q}}$ such that $(\overline{\bar{p}}, \overline{\bar{q}}) \leq\left(p_{i}\left(s_{n+1}, \bar{s}_{i}\right), q_{i}\left(t_{n+1}, \bar{t}_{i}\right)\right)$ and $\left.\left(\left(\bar{s}_{i}, \overline{\bar{p}}\right), \bar{t}_{i}, \overline{\bar{q}}\right)\right) \in \mathbb{A}\left(\mathbb{M}^{2}\right)$. Define

$$
p_{i+1}:=\overline{\bar{p}} \cup\left(p_{i} \backslash p_{i}\left(s_{n+1}, \bar{s}_{i}\right)\right)
$$

and

$$
q_{i+1}:=\overline{\bar{q}} \cup\left(q_{i} \backslash q_{i}\left(t_{n+1}, \bar{t}_{i}\right)\right)
$$

Then clearly we have

$$
\left(\left(\bar{s}_{i}, p_{i+1}\left(s_{n+1}, \bar{s}_{i}\right)\right),\left(\bar{t}_{i}, q_{i+1}\left(t_{n+1}, \bar{t}_{i}\right)\right)\right) \Vdash_{\mathbb{A}\left(\mathbb{M}^{2}\right)} \dot{f}(n+1)=K,
$$

as well.

In the end, define $u:=p_{N}$ and $v:=q_{N}$.

$\square($ Claim 1)

Fix $(u, v) \in \mathbb{M}^{2}$ as in claim 1 and define $H^{\prime}$ to be the collection of all $K$ with $\left(\left(s^{\prime}, u\left(s_{n+1}, s^{\prime}\right)\right),\left(t^{\prime}, v\left(t_{n+1}, t^{\prime}\right)\right)\right) \Vdash_{\left.\mathrm{A}_{(\mathbb{M}}^{2}\right)} \dot{f}(n+1)=K$ for some $\left(s^{\prime}, t^{\prime}\right)$ as in claim 1 , then $H^{\prime}$ is finite and the cardinality of $H^{\prime}$ only depends on the number of $\sigma_{i}$ 's and $\tau_{i}$ ', which we have already chosen, hence on $n$.

Now we thin out $u$ and $v$ such that for every pair $\left(s^{\prime}, t^{\prime}\right)$ as in claim 1 with $\left|s^{\prime}\right|=\left|t^{\prime}\right|$ and every $\rho \in u\left(s_{n+1}, s^{\prime}\right)$ such that $\left(\left(s^{\prime} \frown\langle\rho\rangle, u\right),\left(t^{\prime}, v\right)\right) \in \mathbb{A}\left(\mathbb{M}^{2}\right)$ and for every $\left(s^{\prime}, t^{\prime}\right)$ as in claim 1 with $\left|s^{\prime}\right|=\left|t^{\prime}\right|+1$ and every $\rho \in v\left(t_{n+1}, t^{\prime}\right)$ such that $\left(\left(s^{\prime}, u\right),\left(t^{\prime}\langle\langle\rho, v)) \in \mathbb{A}\left(\mathbb{M}^{2}\right)\right.\right.$, respectively, we have

$$
\min \{|\rho|, \rho(|\mu|)\}>\max \left(\left\{\# \sigma \mid \sigma \in \operatorname{ran}\left(s_{n+1}\right)\right\} \cup\left\{\# \tau \mid \tau \in \operatorname{ran}\left(t_{n+1}\right)\right\}\right),
$$


where $\mu$ is the direct predecessor of $\rho$ in the tree sense in $\operatorname{ran}\left(s^{\prime}\right), \operatorname{ran}\left(t^{\prime}\right)$, respectively. For this, we have to consider all pairs $\left(s^{\prime}, t^{\prime}\right)$ and cut of finitely often finitely many superperfect trees, so there is no problem for the remaining tree to be a superperfect one. This implies the following property for $\left(s^{\prime}, t^{\prime}\right)$ as in claim 1:

(*) Suppose $\rho \in u\left(s_{n+1}, s^{\prime}\right)$ such that $\left(\left(s^{\prime} \frown\langle\rho\rangle, u\right),\left(t^{\prime}, v\right)\right) \in \mathbb{A}\left(\mathbb{M}^{2}\right)$. If $\bar{s} \supseteq s^{\prime} \frown\langle\rho\rangle$ and $\bar{t} \supseteq t^{\prime}$ with $((\bar{s}, u),(\bar{t}, v)) \in \mathbb{A}\left(\mathbb{M}^{2}\right)$ and with $((\bar{s}, u),(\bar{t}, v)) \leq\left(\left(s^{\prime} \frown\langle\rho\rangle, u\right),\left(t^{\prime}, v\right)\right)$, then we have $\bar{s} \subseteq u\left(s_{n+1}, s^{\prime}\right)$ and $\bar{t} \subseteq v\left(t_{n+1}, t^{\prime}\right)$.

And similar suppose $\rho \in v\left(t_{n+1}, t^{\prime}\right)$ such that $\left(\left(s^{\prime}, u\right),\left(t^{\prime} \frown\langle\rho\rangle, v\right)\right) \in$ $\mathbb{A}\left(\mathbb{M}^{2}\right)$. If $\bar{t} \supseteq t^{\prime} \frown\langle\rho\rangle$ and $\bar{s} \supseteq s^{\prime}$ with $((\bar{s}, u),(\bar{t}, v)) \in \mathbb{A}\left(\mathbb{M}^{2}\right)$ and $((\bar{s}, u),(\bar{t}, v)) \leq\left(\left(s^{\prime}, u\right),\left(t^{\prime} \frown\langle\rho\rangle, v\right)\right)$, then we have $\bar{s} \subseteq u\left(s_{n+1}, s^{\prime}\right)$ and $\bar{t} \subseteq v\left(t_{n+1}, t^{\prime}\right)$.

By a similar fusion as by the construction of the pair $\left(p^{\prime}, q^{\prime}\right)$ in the proof of lemma 1.2.1, using the pure decision property of $\mathbb{A}\left(\mathbb{M}^{2}\right)$, we get $\left(u^{\prime}, v^{\prime}\right) \in$ $\mathbb{M}^{2}$ such that $\left(\left(s_{n+1}, u^{\prime}\right),\left(t_{n+1}, v^{\prime}\right)\right) \in \mathbb{A}\left(\mathbb{M}^{2}\right), \quad\left(\left(s_{n+1}, u^{\prime}\right),\left(t_{n+1}, v^{\prime}\right)\right) \leq$ $\left(\left(s_{n+1}, u\right),\left(t_{n+1}, v\right)\right)$ and such that for every $\left(s^{\prime}, t^{\prime}\right) \in<\omega\left(\operatorname{split}\left(u^{\prime}\right)\right) \times<\omega\left(\operatorname{split}\left(v^{\prime}\right)\right)$ with $\operatorname{ran}\left(s^{\prime}\right) \nsubseteq \operatorname{ran}\left(s_{n}\right), \quad\left(\left(s^{\prime}, u^{\prime}\right),\left(t^{\prime}, v^{\prime}\right)\right) \in \mathbb{A}\left(\mathbb{M}^{2}\right)$ and $\left(\left(s^{\prime}, u^{\prime}\right),\left(t^{\prime}, v^{\prime}\right)\right) \leq$ $\left(\left(s, u^{\prime}\right),\left(t, v^{\prime}\right)\right)$, the element $\left(\left(s^{\prime}, u^{\prime}\right),\left(t^{\prime}, v^{\prime}\right)\right)$ decides $\dot{f}(n+1)$.

Claim 2: There exists a pair $\left(u^{\prime \prime}, v^{\prime \prime}\right) \in \mathbb{M}^{2}$ with $\left(u^{\prime \prime}, v^{\prime \prime}\right) \leq\left(u^{\prime}, v^{\prime}\right)$ such that $\left(\left(s_{n+1}, u^{\prime \prime}\right),\left(t_{n+1}, v^{\prime \prime}\right)\right) \in \mathbb{A}\left(\mathbb{M}^{2}\right)$ and for every pair $\left(s^{\prime}, t^{\prime}\right) \in<\omega\left(\operatorname{ran}\left(s_{n+1}\right)\right) \times$ $<\omega\left(\operatorname{ran}\left(t_{n+1}\right)\right)$ with $\left(\left(s^{\prime}, u^{\prime \prime}\right),\left(t^{\prime}, v^{\prime \prime}\right)\right) \in \mathbb{A}\left(\mathbb{M}^{2}\right)$ and $\left(\left(s^{\prime}, u^{\prime \prime}\right),\left(t^{\prime}, v^{\prime \prime}\right)\right) \leq$ $\left(\left(s, u^{\prime}\right),\left(t, v^{\prime}\right)\right)$ we have the following:

If $\tau \in \operatorname{ran}\left(s_{n+1}\right)$ with $\left(\left(s^{\prime}\langle\tau\rangle, u^{\prime \prime}\right),\left(t^{\prime}, v^{\prime \prime}\right)\right) \in \mathbb{A}\left(\mathbb{M}^{2}\right)$ then there exists $K$ such that for every $\rho \in \operatorname{split}\left(u^{\prime \prime}\left(s_{n+1}, s^{\prime}\langle\tau\rangle\right)\right)$ which properly extends $\tau$ we have $\left(\left(s^{\prime}\langle\rho\rangle, u^{\prime \prime}\right),\left(t^{\prime}, v^{\prime \prime}\right)\right) \Vdash_{{\mathbb{A}\left(\mathbb{M}^{2}\right)}^{2}} \dot{f}(n+1)=K$;

if $\tau \in \operatorname{ran}\left(t_{n+1}\right)$ with $\left(\left(s^{\prime}, u^{\prime \prime}\right),\left(t^{\prime} \frown\langle\tau\rangle, v^{\prime \prime}\right)\right) \in \mathbb{A}\left(\mathbb{M}^{2}\right)$ then there exists $K$ such that for every $\rho \in \operatorname{split}\left(v^{\prime \prime}\left(t_{n+1}, t^{\prime} \frown\langle\tau\rangle\right)\right)$ which properly extends $\tau$ we have $\left(\left(s^{\prime}, u^{\prime \prime}\right),\left(t^{\prime}\langle\rho\rangle, v^{\prime \prime}\right)\right) \Vdash_{\mathrm{A}\left(\mathbb{M}^{2}\right)} \dot{f}(n+1)=K$.

Proof of claim 2: Let $\left(\left(\bar{s}_{i}, \bar{t}_{i}, \bar{\tau}_{i}\right)\right)_{i<N}$ for some $N \in \omega$ enumerate all tripel $\left(s^{\prime}, t^{\prime}, \tau\right)$ as in the claim. Define $p_{0}:=u^{\prime}$ and $q_{0}:=v^{\prime}$ and suppose we have constructed $\left(p_{i}, q_{i}\right)$ for some $i<N$. Consider $\left(\bar{s}_{i}, \bar{t}_{i}, \bar{\tau}_{i}\right)$ and suppose $\bar{\tau}_{i} \in \operatorname{ran}\left(s_{n+1}\right)$, so $\left|\bar{s}_{i}\right|=\left|\bar{t}_{i}\right| ;$ the case $\bar{\tau}_{i} \in \operatorname{ran}\left(t_{n+1}\right)$ is similar.

For all $\rho \in \operatorname{split}\left(p_{i}\left(s_{n+1}, \bar{s}_{i} \frown\left\langle\bar{\tau}_{i}\right\rangle\right)\right)$ which properly extends $\bar{\tau}_{i}$ and with $\left(\left(\bar{s}_{i} \frown\langle\rho\rangle, p_{i}\right),\left(\bar{t}_{i}, q_{i}\right)\right) \in \mathbb{A}\left(\mathbb{M}^{2}\right)$ we have $\operatorname{ran}\left(\bar{s}_{i} \frown\langle\rho\rangle\right) \nsubseteq \operatorname{ran}\left(s_{n}\right)$ (by property $(*)),\left(\left(\bar{s}_{i} \frown\langle\rho\rangle, u^{\prime}\right),\left(\bar{t}_{i}, v^{\prime}\right)\right) \in \mathbb{A}\left(\mathbb{M}^{2}\right)$ and $\left(\left(\bar{s}_{i} \frown\langle\rho\rangle, u^{\prime}\right),\left(\bar{t}_{i}, v^{\prime}\right)\right) \leq\left(\left(s, u^{\prime}\right),\left(t, v^{\prime}\right)\right)$. 
The choice of $\left(u^{\prime}, v^{\prime}\right)$ gives us that $\left(\left(\bar{s}_{i} \frown\langle\rho\rangle, p_{i}\right),\left(\bar{t}_{i}, q_{i}\right)\right)$ decides $\dot{f}(n+1)$, say $\left(\left(\bar{s}_{i} \frown\langle\rho\rangle, p_{i}\right),\left(\bar{t}_{i}, q_{i}\right)\right) \Vdash_{\left.\mathrm{A}_{(\mathbb{M}}^{2}\right)} \dot{f}(n+1)=K_{\rho}$.

By colouring splitnodes as in the proof of claim 4 in lemma 1.2.1, we can find for every above considered $\rho \in \operatorname{Succ}_{\left.p_{i}\left(s_{n+1}, \bar{s}_{i} \backslash \bar{\tau}_{i}\right\rangle\right)}\left(\bar{\tau}_{i}\right)$ a $K_{\rho}$ and a tree $p^{\rho} \in \mathbb{M}$ such that $p^{\rho} \subseteq\left(p_{i}\right)_{\rho}$ and for every $\nu \in \operatorname{split}\left(p^{\rho}\right)$ we have $\left(\left(\bar{s}_{i} \frown\langle\nu\rangle, p_{i}\right),\left(\bar{t}_{i}, q_{i}\right)\right) \Vdash_{\mathbb{A}\left(\mathbb{M}^{2}\right)}$ $\dot{f}(n+1)=K_{\rho}$. There are infinitely many $\rho$ but finitely many $K_{\rho}$, so there is an infinite set $A$ of $\rho$ 's and a $K_{i}$ with $K_{\rho}=K_{i}$ for every $\rho \in A$. Fix $\mu \in \operatorname{ran}\left(\bar{s}_{i}\right)$, the direct predecessor in the tree sense of $\bar{\tau}_{i}$ and define

$$
\begin{aligned}
p_{i+1}:=\bigcup\left\{p^{\rho} \mid \rho \in A\right\} \cup\left\{\sigma \in p_{i} \mid \mu \nsubseteq \sigma \vee\right. & \\
& \left.\exists \nu \in \operatorname{ran}\left(s_{n+1}\right)(\mu \varsubsetneqq \nu \subseteq \sigma \vee \mu \subseteq \sigma \varsubsetneqq \nu)\right\}
\end{aligned}
$$

and $q_{i+1}:=q_{i}$.

In the end, define $u^{\prime \prime}:=p_{N}$ and $v^{\prime \prime}:=q_{N}$.

$\square($ Claim 2)

Define $u_{n+1}:=u^{\prime \prime}$ and $v_{n+1}:=v^{\prime \prime}$ and let $H^{\prime \prime}$ be the collection of all $K$ as in claim 2. Clearly, $H^{\prime \prime}$ is finite and the cardinality only depends on $n$. Define $H_{n+1}:=H^{\prime} \cup H^{\prime \prime}$ then we can choose $m_{n+1} \in \omega$ with $m_{n+1}>m_{n}$ and $\left|H^{\prime \prime}\right| \leq 2^{m_{n+1}}$.

Claim 3: $\left(\left(s, u_{n+1}\right),\left(t, v_{n+1}\right)\right) \Vdash_{\left.\mathbb{A}_{(\mathbb{M}}^{2}\right)} \dot{f}(n+1) \in H_{n+1}$.

Proof of claim 3: Let $\left(\left(s^{\prime}, u^{\prime}\right),\left(t^{\prime}, v^{\prime}\right)\right) \in \mathbb{A}\left(\mathbb{M}^{2}\right)$ with $\left(\left(s^{\prime}, u^{\prime}\right),\left(t^{\prime}, v^{\prime}\right)\right) \leq$ $\left(\left(s, u_{n+1}\right),\left(t, v_{n+1}\right)\right)$ and $\left(\left(s^{\prime}, u^{\prime}\right),\left(t^{\prime}, v^{\prime}\right)\right) \Vdash_{\left.\mathrm{A}_{(\mathbb{M}}{ }^{2}\right)} \dot{f}(n+1)=K$ for some $K$. Let $\bar{s}$ be the maximal initial segment of $s^{\prime}$ with $\operatorname{ran}(\bar{s}) \subseteq \operatorname{ran}\left(s_{n+1}\right)$ and $\bar{t}$ the maximal initial segment of $t^{\prime}$ with $\operatorname{ran}(\bar{t}) \subseteq \operatorname{ran}\left(t_{n+1}\right)$. Without loss of generality, the length of $s^{\prime}$ is longer than the length of $\bar{s}$ and the length of $t^{\prime}$ is longer than the length of $\bar{t}$. Define $\mu:=s^{\prime}(|\bar{s}|)$ and $\nu:=t^{\prime}(|\bar{t}|)$. Distinguish the following two cases:

First case: $\mu \in u_{n+1}\left(s_{n+1}, \bar{s}\right)$ and $\nu \in v_{n+1}\left(t_{n+1}, \bar{t}\right)$, so by property $(*)$ we have $\operatorname{ran}\left(s^{\prime}\right) \subseteq u_{n+1}\left(s_{n+1}, \bar{s}\right)$ and $\operatorname{ran}\left(t^{\prime}\right) \subseteq v_{n+1}\left(t_{n+1}, \bar{t}\right)$ and hence $\left(\left(s^{\prime}, u^{\prime}\right),\left(t^{\prime}, v^{\prime}\right)\right)$ and $\left(\left(\bar{s}, u_{n+1}\left(s_{n+1}, \bar{s}\right)\right),\left(\bar{t}, v_{n+1}\left(t_{n+1}, \bar{t}\right)\right)\right)$ are compatible. Since

$$
\left(\left(\bar{s}, u_{n+1}\left(s_{n+1}, \bar{s}\right)\right),\left(\bar{t}, v_{n+1}\left(t_{n+1}, \bar{t}\right)\right)\right) \leq\left(\left(\bar{s}, u\left(s_{n+1}, \bar{s}\right)\right),\left(\bar{t}, v\left(t_{n+1}, \bar{t}\right)\right)\right)
$$

and $\left(\left(\bar{s}, u\left(s_{n+1}, \bar{s}\right)\right),\left(\bar{t}, v\left(t_{n+1}, \bar{t}\right)\right)\right) \Vdash_{\left.\mathrm{A}_{(\mathbb{M}}^{2}\right)} \dot{f}(n+1) \in H^{\prime}$ we conclude that $\left(\left(s^{\prime}, u^{\prime}\right),\left(t^{\prime}, v^{\prime}\right)\right) \Vdash_{\mathrm{A}\left(\mathbb{M}^{2}\right)} \dot{f}(n+1) \in H^{\prime}$.

Second case: $\mu \notin u_{n+1}\left(s_{n+1}, \bar{s}\right)$ or $\nu \notin v_{n+1}\left(t_{n+1}, \bar{t}\right)$. Suppose $\mu \notin u_{n+1}\left(s_{n+1}, \bar{s}\right)$, therefore $\mu$ extends an element of $\operatorname{ran}\left(s_{n+1}\right) \backslash \operatorname{ran}(\bar{s})$; the case $\nu \notin v_{n+1}\left(t_{n+1}, \bar{t}\right)$ is similar. Choose $\sigma \in \operatorname{ran}\left(s_{n+1}\right)$ with maximal length such that $\mu \supsetneqq \sigma$. It is easy to see that we have $\left(\left(\bar{s}^{\prime}\langle\sigma\rangle, u_{n+1}\right),\left(\bar{t}, v_{n+1}\right)\right) \in \mathbb{A}\left(\mathbb{M}^{2}\right)$, hence $(\bar{s}, \bar{t}, \sigma)$ is among the enumerated tripels of claim 2 above, say $(\bar{s}, \bar{t}, \sigma)=\left(\bar{s}_{i}, \bar{t}_{i}, \bar{\tau}_{i}\right)$. Then $\mu$ is an 
element of $\operatorname{split}\left(p_{i}\left(s_{n+1}, \bar{s}_{i} \frown\left\langle\bar{\tau}_{i}\right\rangle\right)\right)$ with $\mu \supsetneqq \bar{\tau}_{i}$ and $\left(\left(\bar{s}_{i} \frown\langle\mu\rangle, p_{i}\right),\left(\bar{t}_{i}, q_{i}\right)\right) \Vdash_{\left.\mathbb{A}_{(\mathbb{M}}{ }^{2}\right)}$ $\dot{f}(n+1)=K_{i} \in H^{\prime \prime}$. Since $\left(\left(s^{\prime}, u^{\prime}\right),\left(t^{\prime}, v^{\prime}\right)\right) \leq\left(\left(\bar{s}_{i} \frown\langle\mu\rangle, p_{i}\right),\left(\bar{t}_{i}, q_{i}\right)\right)$, also $\left(\left(s^{\prime}, u^{\prime}\right),\left(t^{\prime}, v^{\prime}\right)\right) \Vdash_{\mathrm{A}\left(\mathbb{M}^{2}\right)} \dot{f}(n+1) \in H^{\prime \prime}$.

$\square($ claim 3)

If $n$ is even, we have to do the analogous step at the right side by choosing $\tau \in \operatorname{Succ}_{v_{n}}(\nu)$, where $\nu \in \operatorname{ran}\left(t_{n}\right)$ is the node, where we have to extend $\operatorname{ran}\left(t_{n}\right)$. Then we let $t_{n+1}:=t_{n} \frown\langle\tau\rangle$ and $s_{n+1}:=s_{n}$. This step is very similar to the even case, we do not carry it out. This finishes the construction.

In the end, define $p^{\prime}$ and $q^{\prime}$ to be the superperfect trees determined by

$$
\operatorname{split}\left(p^{\prime}\right)=\bigcup\left\{\operatorname{ran}\left(s_{n}\right) \mid n \in \omega\right\}
$$

and

$$
\operatorname{split}\left(q^{\prime}\right)=\bigcup\left\{\operatorname{ran}\left(t_{n}\right) \mid n \in \omega\right\} .
$$

Then $\left(p^{\prime}, q^{\prime}\right)$ and $\left(H_{n}\right)_{n \in \omega}$ are as desired.

It is well known, that the Laver property of a forcing $P$ implies, that forcing with $P$ does not add Cohen-reals.

\subsection{The model}

Definition 1.4.1 Let $G$ be an $\mathbb{A}\left(\mathbb{M}^{2}\right)$-generic filter over $V$. Define $\left(p_{G}, q_{G}\right)$ to be the unique pair $\left(p^{\prime}, q^{\prime}\right) \in \mathbb{M}^{2}$ such that

$$
\operatorname{split}\left(p^{\prime}\right)=\bigcup\left\{\operatorname{ran}(s) \mid \exists p, q \in \mathbb{M} \exists t \in \in^{<\omega}(<\omega \omega)(((s, p),(t, q)) \in G)\right\}
$$

and

$$
\operatorname{split}\left(q^{\prime}\right)=\bigcup\left\{\operatorname{ran}(t) \mid \exists p, q \in \mathbb{M} \exists s \in \in^{<\omega}(<\omega \omega)(((s, p),(t, q)) \in G)\right\} .
$$

Clearly, $p_{G}$ and $q_{G}$ are welldefined.

For justifying the name amoeba for our forcing $\mathbb{A}\left(\mathbb{M}^{2}\right)$ we want to prove the following lemma:

Lemma 1.4.2 Suppose that $D \subseteq \mathbb{M}^{2}$ is open and dense and in $V$ and $G$ is $\mathbb{A}\left(\mathbb{M}^{2}\right)$-generic over $V$.

Then there exists a countable $F \subseteq D$ in $V$ such that in $V[G]$ we have $\left[p_{G}\right] \times\left[q_{G}\right] \subseteq \bigcup\{[p] \times[q] \mid(p, q) \in F\}$.

Moreover, every pair of branches of $\left(p_{G}, q_{G}\right)$ is $\mathbb{M}^{2}$-generic over $V$.

For the proof we use the following: 
Fact 1.4.3 [Sp2] Suppose $D \subseteq \mathbb{M}^{2}$ is open and dense and $(p, q) \in \mathbb{M}^{2}$.

Then there exists $\left(p^{\prime}, q^{\prime}\right) \in \mathbb{M}^{2}$ with $\left(p^{\prime}, q^{\prime}\right) \leq^{0}(p, q)$ and there exists some countable $F \subseteq D$ such that for every $(x, y) \in\left[p^{\prime}\right] \times\left[q^{\prime}\right]$ there is $(u, v) \in F$ with $(x, y) \in[u] \times[v]$.

This fact is the result of a difficult induction on types invented by Spinas.

Proof of lemma 1.4.2: So suppose $G$ is $\mathbb{A}\left(\mathbb{M}^{2}\right)$-generic over $V$ and $D \subseteq \mathbb{M}^{2}$ is open and dense. Define

$$
\begin{aligned}
& \bar{D}:=\left\{\left(p^{\prime}, q^{\prime}\right) \in \mathbb{M}^{2} \mid \text { there exists a countable } F \subseteq D\right. \text { such that } \\
& \left.\left.\qquad\left[p^{\prime}\right] \times\left[q^{\prime}\right] \subseteq \bigcup\{u] \times[v] \mid(u, v) \in F\right\}\right\},
\end{aligned}
$$

then $\bar{D}$ is $\leq^{0}$-dense in $\mathbb{M}^{2}$ by fact 1.4.3; recall that this means that for every $(p, q) \in \mathbb{M}^{2}$ there exists $\left(p^{\prime}, q^{\prime}\right) \leq(p, q)$ with $\left(p^{\prime}, q^{\prime}\right) \in \bar{D}$ and $\operatorname{st}\left(p^{\prime}\right)=\operatorname{st}(p)$ and $\operatorname{st}\left(q^{\prime}\right)=\operatorname{st}(q)$. So, given $((s, p),(t, q)) \in \mathbb{A}\left(\mathbb{M}^{2}\right)$ it is easy to find $\left(p^{\prime}, q^{\prime}\right) \leq 0(p, q)$ such that $\left(\left(s, p^{\prime}\right),\left(t, q^{\prime}\right)\right) \in \mathbb{A}\left(\mathbb{M}^{2}\right)$ and there exists a countable $F \subseteq D$ such that $\left[p^{\prime}\right] \times\left[q^{\prime}\right] \subseteq \bigcup\{[u] \times[v] \mid(u, v) \in F\}$. Hence the set

$$
\begin{aligned}
D^{\prime}:=\left\{\left(\left(s, p^{\prime}\right),\left(t, q^{\prime}\right)\right) \in \mathbb{A}\right. & \left(\mathbb{M}^{2}\right) \mid \\
& \exists F \subseteq D \text { countable } \\
& \left.\left(\left[p^{\prime}\right] \times\left[q^{\prime}\right] \subseteq \bigcup\{[u] \times[v] \mid(u, v) \in F\}\right)\right\}
\end{aligned}
$$

is $\leq^{0}$-dense in $\mathbb{A}\left(\mathbb{M}^{2}\right)$, i.e. for every $((s, p),(t, q)) \in \mathbb{A}\left(\mathbb{M}^{2}\right)$ exists $\left(p^{\prime}, q^{\prime}\right) \leq 0$ $(p, q)$ such that $\left(\left(s, p^{\prime}\right),\left(t, q^{\prime}\right)\right) \in D^{\prime}$. Choose $\left(\left(s, p^{\prime}\right),\left(t, q^{\prime}\right)\right) \in D^{\prime} \cap G$ with $\left(\left(s, p^{\prime}\right),{ }^{\prime}\left(t, q^{\prime}\right)\right) \leq^{0}((s, p),(t, q))$ and let $F \in V$ be the countable witness for this. Notice that the property of $F$ is a $\Pi_{1}^{1}$ one and is therefore absolute for transitive models of ZFC, hence $F$ has the same property in $V[G]$. For every $(x, y) \in$ $\left[p_{G}\right] \times\left[q_{G}\right]$ and every $n \in \omega$ there is an element $\left(\left(s_{n}, p_{n}\right),\left(t_{n}, q_{n}\right)\right) \in G$ such that for every $n \in \omega$ we have $\left(\left(s_{n+1}, p_{n+1}\right),\left(t_{n+1}, q_{n+1}\right)\right) \leq\left(\left(s_{n}, p_{n}\right),\left(t_{n}, q_{n}\right)\right) \leq$ $\left(\left(s, p^{\prime}\right),\left(t, q^{\prime}\right)\right)$ and $x\left\lceil n \in S_{n}\right.$ and $y\left\lceil n \in T_{n}\right.$, where $S_{n}$ and $T_{n}$ are the downward closures of $\operatorname{ran}\left(s_{n}\right), \operatorname{ran}\left(t_{n}\right)$, respectively. Hence $(x, y) \in\left[p^{\prime}\right] \times\left[q^{\prime}\right]$ and therefore $\left[p_{G}\right] \times\left[q_{G}\right] \subseteq \bigcup\{[p] \times[q] \mid(p, q) \in F\}$.

It remains to prove that every pair of branches in $\left(p_{G}, q_{G}\right)$ is $\mathbb{M}^{2}$-generic. For this we have to prove that every pair $(x, y) \in{ }^{\omega} \omega \times{ }^{\omega} \omega$ which has the following property:

(*) for every dense $D \subseteq \mathbb{M}^{2}$ in $V$ there exists $(p, q) \in D$ with $(x, y) \in[p] \times[q]$

is a pair of Miller-reals, i.e. $H:=\left\{(p, q) \in\left(\mathbb{M}^{2}\right)^{V} \mid(x, y) \in[p] \times[q]\right\}$ is $\mathbb{M}^{2}$ generic over $V$.

Clearly, $H$ is upwards closed. Suppose there are incompatible $(p, q),\left(p^{\prime}, q^{\prime}\right)$ in $H$. By Shoenfields absoluteness theorem there are incompatible in $V$, i.e. $[p] \cap\left[p^{\prime}\right]$ or $[q] \cap\left[q^{\prime}\right]$ (or both) does not contain the branches of a Miller tree, without 
loss of generality, $[p] \cap\left[p^{\prime}\right]$ does not. Since $[p] \cap\left[p^{\prime}\right]$ is a closed subset of ${ }^{\omega} \omega$, we can apply a fact of Kechris:

Fact 1.4.4 [Ke] Every closed set $A \subseteq{ }^{\omega} \omega$ is bounded with respect to $\leq{ }^{*}$ or contains the branches of a Miller tree.

Choose by this fact an upper bound $f \in{ }^{\omega} \omega$ of $[p] \cap\left[p^{\prime}\right]$. It is easy to see that the set $E:=\left\{(\bar{p}, \bar{q}) \in \mathbb{M}^{2} \mid \forall h \in[\bar{p}]\left(h \mathbb{Z}^{*} f\right)\right\}$ is dense in $\mathbb{M}^{2}$, so by property (*) of $(x, y)$ there exists $(\bar{p}, \bar{q}) \in E$ with $(x, y) \in[\bar{p}] \times[\bar{q}]$, hence we have $x \not^{*} f$. But by choice $x \in[p] \cap\left[p^{\prime}\right]$, therefore $x \leq^{*} f$, this is a contradiction. Hence $H$ is a filter.

The genericity of $H$ is clear by property $(*)$.

Now we are ready to prove the maintheorem of this chapter:

Proof of theorem 1.1.2: Suppose that $\left(\left(P_{\alpha}\right)_{\alpha \leq \omega_{2}},\left(\dot{Q}_{\alpha}\right)_{\alpha<\omega_{2}}\right)$ is a countable support iteration of $\mathbb{A}\left(\mathbb{M}^{2}\right)$, i.e. for every $\alpha<\omega_{2}, \dot{Q}_{\alpha}$ is a $P_{\alpha}$-name for $\mathbb{A}\left(\mathbb{M}^{2}\right)$ defined in the model $V^{P_{\alpha}}$. Let $G$ be $P_{\omega_{2}}$-generic over $V$.

By lemma 1.3.1, every iterand of our iteration has the Laver property and by a result of Shelah ([Sh], see also [Go]) the Laver property is preserved under countable support iterations. So $P_{\omega_{2}}$ has the Laver property, hence $V[G] \models$ $\operatorname{cov}(\mathcal{M})=\omega_{1}$. It remains to prove, that, in $V[G]$, we have $\operatorname{add}\left(J\left(\mathbb{M}^{2}\right)\right)=\omega_{2}$. So suppose, in $V[G],\left(X_{\alpha}\right)_{\alpha<\omega_{1}}$ is a family of elements of $J\left(\mathbb{M}^{2}\right)$ and let $X:=$ $\bigcup\left\{X_{\alpha} \mid \alpha<\omega_{1}\right\}$. Suppose $(p, q) \in \mathbb{M}^{2}$. We have to find $\left(p^{\prime}, q^{\prime}\right) \leq(p, q)$ in $\mathbb{M}^{2}$ such that $\left(\left[p^{\prime}\right] \times\left[q^{\prime}\right]\right) \cap X=\emptyset$.

For $\alpha<\omega_{1}$ define

$$
D_{\alpha}:=\left\{(u, v) \in \mathbb{M}^{2} \mid([u] \times[v]) \cap X_{\alpha}=\emptyset\right\} .
$$

Clearly, $D_{\alpha}$ is open and dense in $\mathbb{M}^{2}$. Our iteration has countable support, hence for every $\alpha<\omega_{2}$ with $\operatorname{cf}(\alpha)=\omega_{1}$ we have for every $(u, v) \in\left(\mathbb{M}^{2}\right)^{V\left[G_{\alpha}\right]}$ that $(u, v)$ already belongs to $V\left[G_{\beta}\right]$ for some $\beta<\alpha$ (see for example $[\mathrm{Ku}$, ch. VIII, lemma 5.14). With this fact, by a Löwenheim-Skolem-argument, the set

$$
\begin{aligned}
C_{\alpha}:=\left\{\beta<\omega_{2} \mid D_{\alpha} \cap V\left[G_{\beta}\right] \in V\left[G_{\beta}\right]\right. \text { and } \\
\left.\qquad D_{\alpha} \cap V\left[G_{\beta}\right] \text { is open and dense in }\left(\mathbb{M}^{2}\right)^{V\left[G_{\beta}\right]}\right\}
\end{aligned}
$$

is $\omega_{1}$-club in $\omega_{2}$, i.e. it is unbounded and closed under increasing sequences of length $\omega_{1}$. Hence $C:=\bigcap_{\alpha<\omega_{1}} C_{\alpha}$ is $\omega_{1}$-club in $\omega_{2}$, too. But $G$ is generic, hence by the above mentioned lemma of $[\mathrm{Ku}]$ we find $\gamma \in C$ and some $(s, t) \in$ $<\omega_{\text {split }}(p) \times{ }^{<\omega} \operatorname{split}(q)$ such that $((s, p),(t, q)) \in G(\gamma)$, where $G(\gamma)$ is the $\dot{Q}_{\gamma}\left[G_{\gamma}\right]-$ generic filter induced by $G$. By lemma 1.4.2 in $V\left[G_{\gamma+1}\right]$ we have for every $\alpha<\omega_{1}$

$$
\left[p_{G(\gamma)}\right] \times\left[q_{G(\gamma)}\right] \subseteq \bigcup\left\{[u] \times[v] \mid(u, v) \in D_{\alpha}^{\prime} \cap V\left[G_{\gamma}\right]\right\}
$$


for some countable $D_{\alpha}^{\prime} \subseteq D_{\alpha}$. By absolutness this is also true in $V[G]$, hence $\left.V[G]=\left(\left[p_{G(\gamma)}\right)\right] \times\left[q_{G(\gamma)}\right]\right) \cap X=\emptyset$ and as $\left(p_{G(\gamma)}, q_{G(\gamma)}\right) \leq(p, q)$ we are done. 


\section{Chapter 2}

\section{Martin's Axiom and the additivity of $\mathcal{J}\left(\mathbb{M}^{2}\right)$}

In this chapter, we want to prove the following theorem:

Theorem 2.0.5 $\mathrm{MA}(\sigma$-centered $)$ implies that $\operatorname{add}\left(\mathcal{J}\left(\mathbb{M}^{2}\right)\right)$ equals $2^{\omega}$.

Where $\sigma$-centered denotes the class of all forcings which are $\sigma$-centered, where a forcing $P$ is $\sigma$-centered, if it is a countable union of centered sets $P_{n}$, i.e. for $p, q \in P_{n}$ there exists $r \in P_{n}$ with $r \leq p, q$.

We will use the following:

Fact 2.0.6 $[\mathrm{JoSp}]$ For $(p, q) \in \mathbb{M}^{2}$ and $X \in J\left(\mathbb{M}^{2}\right)$ there exists $\left(p^{\prime}, q^{\prime}\right) \leq 0(p, q)$ such that $\left(\left[p^{\prime}\right] \times\left[q^{\prime}\right]\right) \cap X=\emptyset$.

In other words, the set $\left\{\left(p^{\prime}, q^{\prime}\right) \in \mathbb{M}^{2} \mid\left(\left[p^{\prime}\right] \times\left[q^{\prime}\right]\right) \cap X=\emptyset\right\}$ is $\leq^{0}$-dense in $\mathbb{M}^{2}$ for fixed $X \in \mathcal{J}\left(\mathbb{M}^{2}\right)$. This fact was the mainstep in proving that $\mathcal{J}\left(\mathbb{M}^{2}\right)$ is a $\sigma$-ideal.

\subsection{Good sequences}

For the proof of theorem 2.0.5 we need the concept of good sequences and the corresponding sequences of superperfect trees defined in the next definitions:

Definition 2.1.1 Suppose $(p, q) \in \mathbb{M}^{2}$ and $(p, q)$ have the property of $\left(p^{\prime}, q^{\prime}\right)$ formulated in fact 0.0.2 (recall that we always asume this).

Let $I_{(p, q)}:=\operatorname{TP}_{\mathrm{st}(p), \mathrm{st}(q)}(p, q)$, then we have $I_{(p, q)} \subseteq \operatorname{split}(p) \times{ }^{+} \operatorname{split}(q)$. A sequence $\mathcal{A}=\left(A_{(\sigma, \tau)}\right)_{(\sigma, \tau) \in I_{(p, q)}}$ is called good for $(p, q)$ or a $\operatorname{good}(p, q)$-sequence if the following hold for every $(\sigma, \tau) \in I_{(p, q)}$ : 
(i) $A_{(\sigma, \tau)} \subseteq{ }^{<\omega} \omega$ is infinite;

(ii) if $\operatorname{tp}(\sigma, \tau)$ is even, then $\forall \rho \in A_{(\sigma, \tau)}(\rho \supsetneqq \sigma \wedge|\rho|>\rho(|\sigma|))$ and $\forall \rho, \rho^{\prime} \in$ $A_{(\sigma, \tau)}\left(\rho \neq \rho^{\prime} \Rightarrow \rho(|\sigma|) \neq \rho^{\prime}(|\sigma|)\right)$

if $\operatorname{tp}(\sigma, \tau)$ is odd, then $\forall \rho \in A_{(\sigma, \tau)}(\rho \supsetneqq \tau \wedge|\rho|>\rho(|\tau|))$ and $\forall \rho, \rho^{\prime} \in$ $A_{(\sigma, \tau)}\left(\rho \neq \rho^{\prime} \Rightarrow \rho(|\tau|) \neq \rho^{\prime}(|\tau|)\right)$

(iii) $A_{(\sigma, \tau)} \subseteq \operatorname{Sop}_{\mathrm{st}(p), \operatorname{st}(q)}^{p, q}(\sigma, \tau)$;

(iv) if $\operatorname{tp}(\sigma, \tau)$ is even and $\sigma^{\prime}, \sigma^{\prime \prime} \in A_{(\sigma, \tau)}$ are such that $\sigma^{\prime}(|\sigma|)<\sigma^{\prime \prime}(|\sigma|)$, then we have $A_{\left(\sigma^{\prime}, \tau\right)} \supseteq A_{\left(\sigma^{\prime \prime}, \tau\right)}$,

if $\operatorname{tp}(\sigma, \tau)$ is odd and $\tau^{\prime}, \tau^{\prime \prime} \in A_{(\sigma, \tau)}$ are such that $\tau^{\prime}(|\tau|)<\tau^{\prime \prime}(|\tau|)$, then we have $A_{\left(\sigma, \tau^{\prime}\right)} \supseteq A_{\left(\sigma, \tau^{\prime \prime}\right)}$;

(v) if $\operatorname{tp}(\sigma, \tau)$ is even, then $\forall \sigma^{\prime} \in A_{(\sigma, \tau)} \exists \sigma^{\prime \prime} \in A_{(\sigma, \tau)}\left(\sigma^{\prime \prime}(|\sigma|)>\sigma^{\prime}(|\sigma|) \wedge\right.$ $\left.A_{\left(\sigma^{\prime}, \tau\right)} \supsetneqq A_{\left(\sigma^{\prime \prime}, \tau\right)}\right)$,

if $\operatorname{tp}(\sigma, \tau)$ is odd, then $\forall \tau^{\prime} \in A_{(\sigma, \tau)} \exists \tau^{\prime \prime} \in A_{(\sigma, \tau)}\left(\tau^{\prime \prime}(|\tau|)>\tau^{\prime}(|\tau|) \wedge\right.$ $\left.A_{\left(\sigma, \tau^{\prime}\right) \supsetneqq} A_{\left(\sigma, \tau^{\prime \prime}\right)}\right)$.

If $(p, q)$ is clear from context, we sometimes omit the mention of $(p, q)$.

For every good sequence $\mathcal{A}=\left(A_{(\sigma, \tau)}\right)_{(\sigma, \tau) \in I_{(p, q)}}$ there is a corresponding sequence $\left(\left(p_{(\sigma, \tau)}^{\mathcal{A}}, q_{(\sigma, \tau)}^{\mathcal{A}}\right)\right)_{(\sigma, \tau) \in I_{(p, q)}}$ in $\mathbb{M}^{2}$ :

We just describe how to get $\left(p_{(\sigma, \tau)}^{\mathcal{A}}, q_{(\sigma, \tau)}^{\mathcal{A}}\right)$ in the case where $\operatorname{tp}(\sigma, \tau)$ is even, the odd case is similar. So suppose $\operatorname{tp}(\sigma, \tau)$ is even. Define $p_{(\sigma, \tau)}^{\mathcal{A}}$ and $q_{(\sigma, \tau)}^{\mathcal{A}}$ by defining

$$
\begin{aligned}
\operatorname{split}_{n}\left(p_{(\sigma, \tau)}^{\mathcal{A}}\right):=\{\bar{\sigma} \in & \operatorname{split}\left(p_{(\sigma, \tau)}^{\mathcal{A}}\right) \mid \bar{\sigma} \text { has exactly } n \operatorname{different} \\
& \left.\operatorname{predecessors} \text { in the tree sense in } \operatorname{split}\left(p_{(\sigma, \tau)}^{\mathcal{A}}\right)\right\}
\end{aligned}
$$

and

$$
\begin{aligned}
\operatorname{split}_{n}\left(q_{(\sigma, \tau)}^{\mathcal{A}}\right):=\left\{\bar{\tau} \in \operatorname{split}\left(q_{(\sigma, \tau)}^{\mathcal{A}}\right) \mid \bar{\tau} \text { has exactly } n \operatorname{different}\right. \\
\\
\left.\quad \operatorname{predecessors~in~the~tree~sense~in~} \operatorname{split}\left(q_{(\sigma, \tau)}^{\mathcal{A}}\right)\right\}
\end{aligned}
$$

by induction on $n$.

For $n=0$ let $\operatorname{split}_{0}\left(p_{(\sigma, \tau)}^{\mathcal{A}}\right)=\left\{\operatorname{st}\left(p_{(\sigma, \tau)}^{\mathcal{A}}\right)\right\}:=\{\sigma\}$ and $\operatorname{split}_{0}\left(q_{(\sigma, \tau)}^{\mathcal{A}}\right)=$ $\left\{\operatorname{st}\left(q_{(\sigma, \tau)}^{\mathcal{A}}\right)\right\}:=\{\tau\}$. Suppose for $k \leq n$ we have already defined $\operatorname{split}_{k}\left(p_{(\sigma, \tau)}^{\mathcal{A}}\right)$ and $\operatorname{split}_{k}\left(q_{(\sigma, \tau)}^{\mathcal{A}}\right)$. For defining $\operatorname{split}_{n+1}\left(p_{(\sigma, \tau)}^{\mathcal{A}}\right)$ we have to define what is in $\operatorname{Succ}_{p_{(\sigma, \tau)}^{\mathcal{A}}}(\bar{\sigma})$ for every $\bar{\sigma} \in \operatorname{split}_{n}\left(p_{(\sigma, \tau)}^{\mathcal{A}}\right)$ : Let

$$
\operatorname{Succ}_{p_{(\sigma, \tau)}^{\mathcal{A}}}(\bar{\sigma}):=\bigcup\left\{A_{(\bar{\sigma}, t)} \mid t \in \bigcup_{k \leq n} \operatorname{split}_{k}\left(q_{(\sigma, \tau)}^{\mathcal{A}}\right) \wedge \operatorname{tp}_{(\sigma, \tau)}(\bar{\sigma}, t)=2 n\right\}
$$

for every $\bar{\sigma} \in \operatorname{split}_{n}\left(p_{(\sigma, \tau)}^{\mathcal{A}}\right)$. And then define

$$
\begin{aligned}
\operatorname{Succ}_{q_{(\sigma, \tau)}^{\mathcal{A}}}(\bar{\tau}):=\bigcup\left\{A_{(s, \bar{\tau})} \mid s \in \bigcup_{k \leq n+1} \operatorname{split}_{k}\left(p_{(\sigma, \tau)}^{\mathcal{A}}\right) \wedge\right. \\
\left.\operatorname{tp}_{(\sigma, \tau)}(s, \bar{\tau})=2 n+1\right\}
\end{aligned}
$$


for every $\bar{\tau} \in \operatorname{split}_{n}\left(q_{(\sigma, \tau)}^{\mathcal{A}}\right)$.
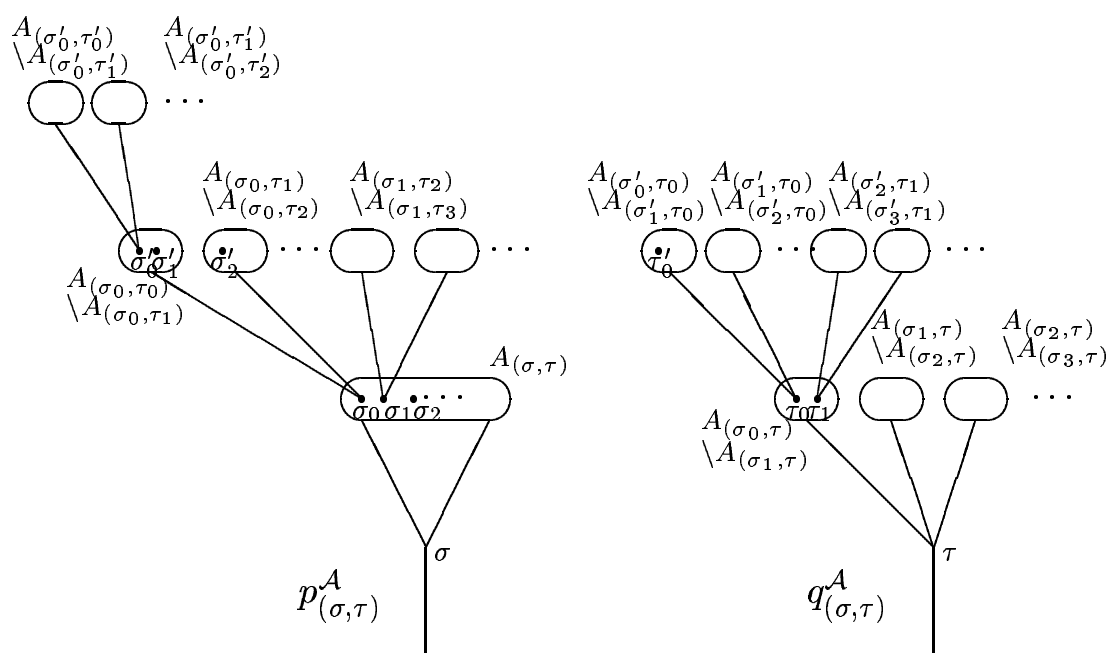

Notice that $\left(p_{(\sigma, \tau)}^{\mathcal{A}}, q_{(\sigma, \tau)}^{\mathcal{A}}\right) \leq(p, q)$, even $\left(p_{(\sigma, \tau)}^{\mathcal{A}}, q_{(\sigma, \tau)}^{\mathcal{A}}\right) \leq 0\left((p)_{\sigma},(q)_{\tau}\right)$, for every $(\sigma, \tau) \in I_{(p, q)}$.

Remark 2.1.2 Fix $(p, q) \in \mathbb{M}^{2}$. Let $\left(p^{\prime}, q^{\prime}\right) \in \mathbb{M}^{2}$ with $\left(p^{\prime}, q^{\prime}\right) \leq(p, q)$ and such that for almost all $n \in \omega$ and every $(x, y) \in\left[p^{\prime}\right] \times^{+}\left[q^{\prime}\right]$ we have tp-npair $(x, y) \in \operatorname{split}\left(p^{\prime}\right) \times \operatorname{split}\left(q^{\prime}\right)$ (recall that for every $(u, v) \in \mathbb{M}^{2},(u, v) \leq(p, q)$, there exists $\left(p^{\prime}, q^{\prime}\right) \leq^{0}(u, v)$ with this property (compare the preliminaries)). Then we can define a partial good sequence $\mathcal{A}=\left(A_{(\sigma, \tau)}\right)_{(\sigma, \tau) \in I^{\prime}}$ for $(p, q)$, where $I^{\prime}:=I_{(p, q)} \cap\left(\operatorname{split}\left(p^{\prime}\right) \times \operatorname{split}\left(q^{\prime}\right)\right):$ For $(\sigma, \tau) \in I^{\prime}$ with $\operatorname{tp}(\sigma, \tau)$ even let $A_{(\sigma, \tau)}$ be the subset of $\operatorname{Succ}_{p^{\prime}}(\sigma) \cap\{\rho \mid \rho(|\sigma|)>\# \sigma\}$ thinned out such that for every $\rho \in A_{(\sigma, \tau)}$ we have $|\rho|>\rho(|\sigma|), \min \{\rho(|\sigma|),|\rho|\}>\# \tau$ and $\rho(|\sigma|)>\# \rho^{\prime}$ for every $\rho^{\prime} \in A_{(\sigma, \tau)}$ with $\rho^{\prime}(|\sigma|)<\rho(|\sigma|)$. Analogously, if $\operatorname{tp}(\sigma, \tau)$ is odd let $A_{(\sigma, \tau)}$ be the subset of $\operatorname{Succ}_{q^{\prime}}(\tau) \cap\{\rho \mid \rho(|\tau|)>\# \tau\}$ thinned out such that for every $\rho \in A_{(\sigma, \tau)}$ we have $|\rho|>\rho(|\tau|), \min \{\rho(|\tau|),|\rho|\}>\# \sigma$ and $\rho(|\tau|)>\# \rho^{\prime}$ for every $\rho^{\prime} \in A_{(\sigma, \tau)}$ with $\rho^{\prime}(|\tau|)<\rho(|\tau|)$. It is easy to see that (i)-(v) of definition 2.1.1 hold for $\mathcal{A}=\left(A_{(\sigma, \tau)}\right)_{(\sigma, \tau) \in I^{\prime}}$.

Definition 2.1.3 Fix $(p, q) \in \mathbb{M}^{2}$. We define the following relations for any $\operatorname{good}(p, q)$-sequences $\mathcal{A}=\left(A_{(\sigma, \tau)}\right)_{(\sigma, \tau) \in I_{(p, q)}}$ and $\mathcal{B}=\left(B_{(\sigma, \tau)}\right)_{(\sigma, \tau) \in I_{(p, q)}}$ :

$$
\begin{aligned}
\mathcal{A} \leq \mathcal{B}: \Leftrightarrow & \left(p_{(\sigma, \tau)}^{\mathcal{A}}, q_{(\sigma, \tau)}^{\mathcal{A}}\right) \leq\left(p_{(\sigma, \tau)}^{\mathcal{B}}, q_{(\sigma, \tau)}^{\mathcal{B}}\right) \text { for every }(\sigma, \tau) \in I_{(p, q)} . \\
\mathcal{A} \approx \mathcal{B}: \Leftrightarrow & A_{(\sigma, \tau)}={ }^{*} B_{(\sigma, \tau)} \text { for every }(\sigma, \tau) \in I_{(p, q)} \text { and } \\
& A_{(\sigma, \tau)}=B_{(\sigma, \tau)} \text { for all but finitely many }(\sigma, \tau) \in I_{(p, q)} . \\
\mathcal{A} \leq{ }^{*} \mathcal{B}: \Leftrightarrow & \text { there exists a good sequence } \mathcal{C} \text { such that } \mathcal{A} \approx \mathcal{C} \leq \mathcal{B} .
\end{aligned}
$$


The good sequence $\mathcal{C}$ in the definition of $\mathcal{A} \leq^{*} \mathcal{B}$ we oftenly call a witness for $\mathcal{A} \leq^{*} \mathcal{B}$. Notice that $\mathcal{A} \leq^{*} \mathcal{B}$ implies $A_{(\sigma, \tau)} \subseteq{ }^{*} B_{(\sigma, \tau)}$ for every $(\sigma, \tau) \in I_{(p, q)}$. Clearly, $\approx$ is an equivalence relation on the set of good sequences for $(p, q)$. We have the following long but elementary lemma:

Lemma 2.1.4 Suppose $(p, q) \in \mathbb{M}^{2}, \mathcal{C}$ is good for $(p, q)$ and $(\sigma, \tau) \in I_{(p, q)}$.

(a) Suppose $\mu \in \operatorname{split}\left(p_{(\sigma, \tau)}^{\mathcal{C}}\right)$ with $\mu \supsetneqq \sigma$. Then we have the following: If $\operatorname{tp}(\sigma, \tau)$ is even, there exist sequences

$$
\mu=s_{0} \supsetneqq \cdots \supsetneqq s_{k}=\sigma \quad \text { and } \quad t_{1} \supsetneqq \cdots \supsetneqq t_{k}=\tau
$$

such that $s_{i} \in C_{\left(s_{i+1}, t_{i+1}\right)}$ for $i \in\{0, \ldots, k-1\}$ and $t_{i} \in C_{\left(s_{i}, t_{i+1}\right)}$ for $i \in\{1, \ldots, k-1\}$. If we choose $t_{1} \prec$-maximal with $\mu \in C_{\left(s_{1}, t_{1}\right)}$ (notice there exists only finitely many $t$ with $\left.\mu \in C_{\left(s_{1}, t\right)}\right)$, where $s_{1}$ is the direct predecessor in the tree sense of $\mu$ in $\operatorname{split}\left(p_{(\sigma, \tau)}^{\mathcal{C}}\right)$, then the sequences are unique.

If $\operatorname{tp}(\sigma, \tau)$ is odd, there exist sequences

$$
\mu=s_{0} \supsetneqq \cdots \supsetneqq s_{k}=\sigma \quad \text { and } \quad t_{1} \supsetneqq \cdots \supsetneqq t_{k+1}=\tau
$$

such that $s_{i} \in C_{\left(s_{i+1}, t_{i+1}\right)}$ for $i \in\{0, \ldots, k-1\}$ and $t_{i} \in C_{\left(s_{i}, t_{i+1}\right)}$ for $i \in\{1, \ldots, k\}$. Again, if we choose $t_{1} \prec$-maximal with $\mu \in C_{\left(s_{1}, t_{1}\right)}$, where $s_{1}$ is the direct predecessor in the tree sense of $\mu$ in $\operatorname{split}\left(p_{(\sigma, \tau)}^{\mathcal{C}}\right)$, then the sequences are unique.

(b) Suppose $\nu \in \operatorname{split}\left(q_{(\sigma, \tau)}^{\mathcal{C}}\right)$ with $\nu \supsetneqq \tau$. Then we have the following: If $\operatorname{tp}(\sigma, \tau)$ is even, there exist sequences

$$
s_{0} \supsetneqq \cdots \supsetneqq s_{k}=\sigma \quad \text { and } \quad \nu=t_{0} \supsetneqq \ldots \supsetneqq t_{k}=\tau
$$

such that $t_{i} \in C_{\left(s_{i}, t_{i+1}\right)}$ and $s_{i} \in C_{\left(s_{i+1}, t_{i+1}\right)}$ for $i \in\{0, \ldots, k-1\}$. If we choose $s_{0} \prec$-maximal with $\nu \in C_{\left(s_{0}, t_{1}\right)}$, where $t_{1}$ is the direct predecessor in the tree sense of $\nu$ in $\operatorname{split}\left(q_{(\sigma, \tau)}^{\mathcal{C}}\right)$, then the sequences are unique.

If $\operatorname{tp}(\sigma, \tau)$ is odd, there exist sequences

$$
s_{0} \supsetneqq \cdots \supsetneqq s_{k}=\sigma \quad \text { and } \quad \nu=t_{0} \supsetneqq \cdots \supsetneqq t_{k+1}=\tau
$$

such that $t_{i} \in C_{\left(s_{i}, t_{i+1}\right)}$ for $i \in\{0, \ldots, k\}$ and $s_{i} \in C_{\left(s_{i+1}, t_{i+1}\right)}$ for $i \in$ $\{0, \ldots, k-1\}$. Again, if we choose $s_{0} \prec$-maximal with $\nu \in C_{\left(s_{0}, t_{1}\right)}$, where $t_{1}$ is the direct predecessor in the tree sense of $\nu$ in $\operatorname{split}\left(q_{(\sigma, \tau)}^{\mathcal{C}}\right)$, then the sequences are unique.

Proof: We only prove the first case of (a), the rest is similar. So suppose that $\operatorname{tp}(\sigma, \tau)$ is even. Let $s_{0}:=\mu$ and choose $n \in \omega$ such that $s_{0}\lceil n$ is the direct predecessor in the tree sense of $s_{0}$ in $\operatorname{split}\left(p_{(\sigma, \tau)}^{\mathcal{C}}\right)$. Then there exists $t \in \operatorname{split}\left(q_{(\sigma, \tau)}^{\mathcal{C}}\right)$ 
with $\operatorname{tp}\left(s_{0}\lceil n, t)\right.$ is even and $s_{0} \in C_{\left(s_{0} \mid n, t\right)}$. Choose the $\prec$-maximal $t$ with that property (property $(\mathrm{v})$ of the definition of good sequences ensures that there are only finitely many such $t$ ) and define $s_{1}:=s_{0}\left\lceil n\right.$ and $t_{1}:=t$.

Suppose now, $s_{j}$ and $t_{j}$ are defined for some $j \in \omega$. If $s_{j}=\sigma$ we have $t_{j}=\tau$ and $k=j$ and we are done. Otherwise, there exists $n \in \omega$ and $m \in \omega$ such that $t_{j} \in C_{\left(s_{j}, t_{j} \mid m\right)}$ and $s_{j} \in C_{\left(s_{j}\left|n, t_{j}\right| m\right)}$. Define $s_{j+1}:=s_{j}\left\lceil n\right.$ and $t_{j+1}:=t_{j}\lceil m$. After finitely many steps the construction stops.

Lemma 2.1.5 Suppose $(p, q) \in \mathbb{M}^{2}$ and $\mathcal{A}$ and $\mathcal{B}$ are good $(p, q)$-sequences. If there exists a good $(p, q)$-sequence $\mathcal{C}$ with $\mathcal{A} \leq \mathcal{C} \approx \mathcal{B}$, then there exists a good $(p, q)$-sequence $\mathcal{D}$ with $\mathcal{A} \approx \mathcal{D} \leq \mathcal{B}$, hence we have $\mathcal{A} \leq{ }^{*} \mathcal{B}$. Moreover, $\mathcal{D}$ can be chosen such that $\mathcal{D} \leq \mathcal{A}$.

Proof: Suppose $\mathcal{A}, \mathcal{B}$ and $\mathcal{C}$ are good $(p, q)$-sequences with $\mathcal{A} \leq \mathcal{C} \approx \mathcal{B}$. Define

$$
D_{(\sigma, \tau)}:= \begin{cases}A_{(\sigma, \tau)} \cap \operatorname{split}\left(p_{(\sigma, \tau)}^{\mathcal{B}}\right), & \text { if } \operatorname{tp}(\sigma, \tau) \text { is even, } \\ A_{(\sigma, \tau)} \cap \operatorname{split}\left(q_{(\sigma, \tau)}^{\mathcal{B}}\right), & \text { if } \operatorname{tp}(\sigma, \tau) \text { is odd. }\end{cases}
$$

Claim: $\mathcal{D}:=\left(D_{(\sigma, \tau)}\right)_{(\sigma, \tau) \in I_{(p, q)}}$ is a good $(p, q)$-sequence such that $\mathcal{A} \approx \mathcal{D} \leq \mathcal{B}$.

Proof of the claim: For proving that $\mathcal{D}$ is good it is sufficient to show $D_{(\sigma, \tau)}={ }^{*} A_{(\sigma, \tau)}$ for every $(\sigma, \tau) \in I_{(p, q)}$.

If $\operatorname{tp}(\sigma, \tau)$ is even, we prove $\left|A_{(\sigma, \tau)} \backslash \operatorname{split}\left(p_{(\sigma, \tau)}^{\mathcal{B}}\right)\right|<\omega$ : For fixed $\mu \in A_{(\sigma, \tau)} \backslash$ $\operatorname{split}\left(p_{(\sigma, \tau)}^{\mathcal{B}}\right)$, therefore $\mu \in \operatorname{split}\left(p_{(\sigma, \tau)}^{\mathcal{C}}\right)$, we have the unique sequences $\mu=$ $s_{0} \supsetneqq \cdots \supsetneqq s_{k}=\sigma$ and $t_{1} \supsetneqq \ldots \supsetneqq t_{k}=\tau$ as in lemma 2.1.4. By choice, there must exist $i \in\{0, \ldots, k\}$ such that $s_{i} \in C_{\left(s_{i+1}, t_{i+1}\right)} \backslash B_{\left(s_{i+1}, t_{i+1}\right)}$. Define $\Phi_{(\sigma, \tau)}(\mu)$ to be the shortest such $s_{i}$. Then $\Phi_{(\sigma, \tau)}\left\lceil\left(A_{(\sigma, \tau)} \backslash \operatorname{split}\left(p_{(\sigma, \tau)}^{\mathcal{B}}\right)\right)\right.$ is an injective function, because for $\mu, \mu^{\prime} \in A_{(\sigma, \tau)}$ we have $\mu(|\sigma|) \neq \mu^{\prime}(|\sigma|)$. So we have $\left|A_{(\sigma, \tau)} \backslash \operatorname{split}\left(p_{(\sigma, \tau)}^{\mathcal{B}}\right)\right| \leq \Sigma_{(s, t) \in I_{(p, q)}}\left|C_{(s, t)} \backslash B_{(s, t)}\right|<\omega$, the last inequality holds because we have $\mathcal{B} \approx \mathcal{C}$, and therefore $B_{(\sigma, \tau)}=C_{(\sigma, \tau)}$ for almost every $(\sigma, \tau) \in I_{(p, q)}$.

If $\operatorname{tp}(\sigma, \tau)$ is odd, we get $\left|A_{(\sigma, \tau)} \backslash \operatorname{split}\left(q_{(\sigma, \tau)}^{\mathcal{B}}\right)\right|<\omega$ by a similar proof.

It remains to prove that we have $A_{(\sigma, \tau)}=D_{(\sigma, \tau)}$ for all but finitely many $(\sigma, \tau) \in I_{(p, q)}$. Define

$$
E:=\left\{(s, t) \in\left({ }^{<\omega} \omega\right)^{2} \mid \exists(\sigma, \tau) \in I_{(p, q)}\left((\sigma, \tau) \geq(s, t) \wedge B_{(\sigma, \tau)} \neq C_{(\sigma, \tau)}\right)\right\} .
$$

$E$ is finite and for all $(s, t)$ in $I_{(p, q)} \backslash E$ we have $\left(p_{(s, t)}^{\mathcal{B}}, q_{(s, t)}^{\mathcal{B}}\right)=\left(p_{(s, t)}^{\mathcal{C}}, q_{(s, t)}^{\mathcal{C}}\right)$, hence $A_{(s, t)}=D_{(s, t)}$.

Corollary 2.1.6 Fix $(p, q) \in \mathbb{M}^{2}$. Then our relation $\leq^{*}$ is transitive on the set of good $(p, q)$-sequences. 
Proof: Suppose $\mathcal{A} \leq^{*} \mathcal{B} \leq^{*} \mathcal{C}$, so there exist good sequences $\mathcal{A}^{\prime}$ and $\mathcal{B}^{\prime}$ witnessing these. Hence we have the following relationships:

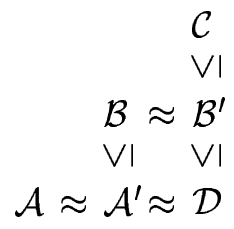

$\mathcal{D}$ exists by lemma 2.1 .5 and is a witness for $\mathcal{A} \leq^{*} \mathcal{C}$.

Lemma 2.1.7 Suppose $(p, q) \in \mathbb{M}^{2}, \mathcal{A}$ is a good $(p, q)$-sequence and $X \in$ $J\left(\mathbb{M}^{2}\right)$. Then there exists a good $(p, q)$-sequence $\mathcal{B} \leq \mathcal{A}$ such that $\left(\left[p_{(\sigma, \tau)}^{\mathcal{B}}\right] \times\right.$ $\left.\left[q_{(\sigma, \tau)}^{\mathcal{B}}\right]\right) \cap X=\emptyset$ for every $(\sigma, \tau) \in I_{(p, q)}$.

Proof: Let $\left(\left(\sigma_{n}, \tau_{n}\right)\right)_{n \in \omega}$ enumerate all pairs in $I_{(p, q)}$ such that $\left(\sigma_{0}, \tau_{0}\right)=$ $(\operatorname{st}(p), \operatorname{st}(q))$. Let $n_{0}:=0$ and choose by fact $2.0 .6\left(p_{\left(\sigma_{n_{0}}, \tau_{n_{0}}\right)}, q_{\left(\sigma_{n_{0}}, \tau_{n_{0}}\right)}\right)$ in $\mathbb{M}^{2}$ such that $\left(p_{\left(\sigma_{n_{0}}, \tau_{n_{0}}\right)}, q_{\left(\sigma_{n_{0}}, \tau_{n_{0}}\right)}\right) \leq^{0}\left(p_{\left(\sigma_{n_{0}}, \tau_{n_{0}}\right)}^{\mathcal{A}}, q_{\left(\sigma_{n_{0}}, \tau_{n_{0}}\right)}^{\mathcal{A}}\right)$ and

$$
\left(\left[p_{\left(\sigma_{n_{0}}, \tau_{n_{0}}\right)}\right] \times\left[q_{\left(\sigma_{n_{0}}, \tau_{n_{0}}\right)}\right]\right) \cap X=\emptyset .
$$

By a remark in the preliminaries, there exists $\left(p_{\left(\sigma_{n_{0}}, \tau_{n_{0}}\right)}^{\prime}, q_{\left(\sigma_{n_{0}}, \tau_{n_{0}}\right)}^{\prime}\right) \in \mathbb{M}^{2}$ such that $\left(p_{\left(\sigma_{n_{0}}, \tau_{n_{0}}\right)}^{\prime}, q_{\left(\sigma_{n_{0}}, \tau_{n_{0}}\right)}^{\prime}\right) \leq 0 \quad\left(p_{\left(\sigma_{n_{0}}, \tau_{n_{0}}\right)}, q_{\left(\sigma_{n_{0}}, \tau_{n_{0}}\right)}\right)$ and for almost all $n \in \omega$ and every $(x, y) \in\left[p_{\left(\sigma_{n_{0}}, \tau_{n_{0}}\right)}^{\prime}\right] \times^{+}\left[q_{\left(\sigma_{n_{0}}, \tau_{n_{0}}\right)}^{\prime}\right]$ we have $\operatorname{tp}_{\left(\sigma_{n_{0}}, \tau_{n_{0}}\right)}-n$-pair $(x, y) \in$ $\operatorname{split}\left(p_{\left(\sigma_{n_{0}}, \tau_{n_{0}}\right)}^{\prime}\right) \times \operatorname{split}\left(q_{\left(\sigma_{n_{0}}, \tau_{n_{0}}\right)}^{\prime}\right)$. By remark 2.1.2 we get a partial good sequence $\mathcal{B}_{0}=\left(B_{(\sigma, \tau)}^{0}\right)_{(\sigma, \tau) \in I_{0}}$ with $I_{0}:=I_{(p, q)} \cap\left(\operatorname{split}\left(p_{\left(\sigma_{n_{0}}, \tau_{n_{0}}\right)}^{\prime}\right) \times \operatorname{split}\left(q_{\left(\sigma_{n_{0}}, \tau_{n_{0}}\right)}^{\prime}\right)\right)$. Suppose we have already constructed a strictly ascending sequence $\left(n_{i}\right)_{i \leq k}$ of natural numbers and a sequence $\left(\mathcal{B}_{i}\right)_{i \leq k}$ of partial good sequences $\mathcal{B}_{i}=$ $\left(B_{(\sigma, \tau)}^{i}\right)_{(\sigma, \tau) \in I_{i}}$ such that $I_{0} \subseteq \ldots \subseteq I_{k-1} \subseteq I_{k}$ and $\mathcal{B}_{i+1}$ extends the sequence $\mathcal{B}_{i}$ for every $i<k$, this means $B_{(\sigma, \tau)}^{i+1}=B_{(\sigma, \tau)}^{i}$ for every $(\sigma, \tau) \in I_{i}$. Choose $n_{k+1} \in \omega$ minimal such that $\left(\sigma_{n_{k+1}}, \tau_{n_{k+1}}\right) \notin I_{k}$. Choose, by remark 2.0.6, $\left(p_{\left(\sigma_{n_{k+1}}, \tau_{n_{k+1}}\right)}, q_{\left(\sigma_{n_{k+1}}, \tau_{n_{k+1}}\right)}\right)$ in $\mathbb{M}^{2}$ with $\left(p_{\left(\sigma_{n_{k+1}}, \tau_{n_{k+1}}\right)}, q_{\left(\sigma_{n_{k+1}}, \tau_{n_{k+1}}\right)}\right) \leq 0$ $\left(p_{\left(\sigma_{n_{k+1}}, \tau_{n_{k+1}}\right)}^{\mathcal{A}}, q_{\left(\sigma_{n_{k+1}}, \tau_{n_{k+1}}\right)}^{\mathcal{A}}\right)$ and

$$
\left(\left[p_{\left(\sigma_{n_{k+1}}, \tau_{n_{k+1}}\right)}\right] \times\left[q_{\left(\sigma_{n_{k+1}}, \tau_{n_{k+1}}\right)}\right]\right) \cap X=\emptyset .
$$

Thin out $p_{\left(\sigma_{n_{k+1}}, \tau_{n_{k+1}}\right)}$ and $q_{\left(\sigma_{n_{k+1}}, \tau_{n_{k+1}}\right)}$ such that whenever $\sigma_{n_{k+1}} \in p_{\left(\sigma_{n_{i}}, \tau_{n_{i}}\right)} \backslash$ $\operatorname{split}\left(p_{\left(\sigma_{n_{i}}, \tau_{n_{i}}\right)}\right)$ for some $i \leq k$ we have $p_{\left(\sigma_{n_{k+1}}, \tau_{n_{k+1}}\right)} \cap p_{\left(\sigma_{n_{i}}, \tau_{n_{i}}\right)}=\emptyset$ and whenever $\tau_{n_{k+1}} \in q_{\left(\sigma_{n_{i}}, \tau_{n_{i}}\right)} \backslash \operatorname{split}\left(q_{\left(\sigma_{n_{i}}, \tau_{n_{i}}\right)}\right)$ for some $i \leq k$ we have $q_{\left(\sigma_{n_{k+1}}, \tau_{n_{k+1}}\right)} \cap$ $q_{\left(\sigma_{n_{i}}, \tau_{n_{i}}\right)}=\emptyset$ (Notice that we only have to cut out one single Miller tree for every relevant $i$ ). Again by the remark in the preliminaries there exists 
$\left(p_{\left(\sigma_{n_{k+1}}, \tau_{n_{k+1}}\right)}^{\prime}, q_{\left(\sigma_{n_{k+1}}, \tau_{n_{k+1}}\right)}^{\prime}\right) \leq^{0}\left(p_{\left(\sigma_{n_{k+1}}, \tau_{n_{k+1}}\right)}, q_{\left(\sigma_{n_{k+1}}, \tau_{n_{k+1}}\right)}\right)$ such that for almost all $n \in \omega$ and every $(x, y) \in\left[\left(p_{\left(\sigma_{n_{k+1}}, \tau_{n_{k+1}}\right)}^{\prime}\right] \times^{+}\left[q_{\left(\sigma_{n_{k+1}}, \tau_{n_{k+1}}\right)}^{\prime}\right)\right.$ we have $\operatorname{tp}_{\left(\sigma_{n_{k+1}}, \tau_{n} n_{k+1}\right)}-n$-pair $(x, y) \in \operatorname{split}\left(p_{\left(\sigma_{n_{k+1}}, \tau_{n_{k+1}}\right)}^{\prime}\right) \times \operatorname{split}\left(q_{\left(\sigma_{n_{k+1}}, \tau_{n_{k+1}}\right)}^{\prime}\right)$. By remark 2.1.2 there exists a partial good sequence $\mathcal{C}=\left(C_{(\sigma, \tau)}\right)_{(\sigma, \tau) \in J}$ for the pair $\left(p_{\left(\sigma_{n_{k+1}}, \tau_{n_{k+1}}\right)}, q_{\left(\sigma_{n_{k+1}}, \tau_{n_{k+1}}\right)}\right)$, where $J:=I_{(p, q)} \cap\left(\operatorname{split}\left(p_{\left(\sigma_{n_{k+1}}, \tau_{n_{k+1}}\right)}^{\prime}\right) \times\right.$ $\operatorname{split}\left(q_{\left(\sigma_{n_{k+1}}, \tau_{n_{k+1}}\right)}^{\prime}\right)$.

Define $I_{k+1}:=I_{k} \cup J$ and $\mathcal{B}_{k+1}$ by

$$
B_{(\sigma, \tau)}^{k+1}:= \begin{cases}B_{(\sigma, \tau)}^{k}, & \text { if }(\sigma, \tau) \in I_{k}, \\ C_{(\sigma, \tau)}, & \text { if }(\sigma, \tau) \in I_{k+1} \backslash I_{k} .\end{cases}
$$

It is easy to see that $\mathcal{B}_{k+1}$ is a partial good sequence which extends $\mathcal{B}_{k}$.

In the end, we get a good sequence $\mathcal{B}=\left(B_{(\sigma, \tau)}\right)_{(\sigma, \tau) \in \bigcup_{n \in \omega} I_{n}}$. It is $\bigcup_{n \in \omega} I_{n}=$ $I_{(p, q)}$, because for every $(\sigma, \tau) \in I_{(p, q)}$ there exists $n_{k} \in \omega$ with $(\sigma, \tau) \in$ $\operatorname{split}\left(p_{\left(\sigma_{n_{k}}, \tau_{n_{k}}\right)}\right) \times \operatorname{split}\left(q_{\left(\sigma_{n_{k}}, \tau_{n_{k}}\right)}\right)$, hence $B_{(\sigma, \tau)}$ is defined. Clearly, we have $\left(\left[p_{(\sigma, \tau)}^{\mathcal{B}}\right] \times\left[q_{(\sigma, \tau)}^{\mathcal{B}}\right]\right) \cap X=\emptyset$ for every $(\sigma, \tau) \in I_{(p, q)}$.

Lemma 2.1.8 Suppose $\mathrm{MA}_{\kappa}(\sigma$-centered $)$ is true and fix $(p, q) \in \mathbb{M}^{2}$.

If $\left(\mathcal{A}^{\alpha}\right)_{\alpha<\kappa}$ is a $\leq^{*}$-descending sequence of good $(p, q)$-sequences, then there exists a good $(p, q)$-sequence $\mathcal{B}$ such that $\mathcal{B} \leq^{*} \mathcal{A}^{\alpha}$ for all $\alpha<\kappa$.

Proof: Define a forcing $P$ as follows:

Conditions in $P$ are of the form $\left(F,\left\{T_{(\sigma, \tau)} \mid(\sigma, \tau) \in S\right\}\right)$ such that

(a) $F$ is a finite subset of $\kappa$;

(b) $S$ is a finite subset of $I_{(p, q)}$;

(c) $T_{(\sigma, \tau)}$ is a finite subset of $<\omega \omega$;

(d) if $\operatorname{tp}(\sigma, \tau)$ is even, then $\forall s \in T_{(\sigma, \tau)}(s \supsetneqq \sigma \wedge|s|>s(|\sigma|))$ and $\forall s, s^{\prime} \in$ $T_{(\sigma, \tau)}\left(s \neq s^{\prime} \Rightarrow s(|\sigma|) \neq s^{\prime}(|\sigma|)\right)$,

if $\operatorname{tp}(\sigma, \tau)$ is odd, then $\forall t \in T_{(\sigma, \tau)}(t \supsetneqq \tau \wedge|t|>t(|\tau|))$ and $\forall t, t^{\prime} \in$ $T_{(\sigma, \tau)}\left(t \neq t^{\prime} \Rightarrow t(|\tau|) \neq t^{\prime}(|\tau|)\right)$;

(e) $T_{(\sigma, \tau)} \subseteq \operatorname{Sop}_{\mathrm{st}(p), \operatorname{st}(q)}^{(p, q)}(\sigma, \tau)$;

(f) if $\operatorname{tp}(\sigma, \tau)$ is even and $(\sigma, \tau),(\rho, \tau),\left(\rho^{\prime}, \tau\right)$ are in $S$ such that $\rho, \rho^{\prime} \in T_{(\sigma, \tau)}$ and $\rho(|\sigma|)<\rho^{\prime}(|\sigma|)$, then we have $T_{(\rho, \tau)} \supseteq T_{\left(\rho^{\prime}, \tau\right)}$;

if $\operatorname{tp}(\sigma, \tau)$ is odd and $(\sigma, \tau),(\sigma, \rho),\left(\sigma, \rho^{\prime}\right)$ are in $S$ such that $\rho, \rho^{\prime} \in T_{(\sigma, \tau)}$ and $\rho(|\tau|)<\rho^{\prime}(|\tau|)$, then we have $T_{(\sigma, \rho)} \supseteq T_{\left(\sigma, \rho^{\prime}\right)}$. 
Define $\left(F,\left\{T_{(\sigma, \tau)} \mid(\sigma, \tau) \in S\right\}\right) \leq\left(F^{\prime},\left\{T_{(\sigma, \tau)}^{\prime} \mid(\sigma, \tau) \in S^{\prime}\right\}\right): \Leftrightarrow$

$$
\begin{aligned}
& F \supseteq F^{\prime}, \\
& S \supseteq S^{\prime}, \\
& \forall(\sigma, \tau) \in S^{\prime}\left(T_{(\sigma, \tau)} \supseteq T_{(\sigma, \tau)}^{\prime}\right) \text { and } \\
& \forall(\sigma, \tau) \in S \forall \alpha \in F^{\prime}\left(T_{(\sigma, \tau)} \backslash T_{(\sigma, \tau)}^{\prime} \subseteq \operatorname{split}\left(r_{(\sigma, \tau)}^{\mathcal{A}^{\alpha}}\right)\right),
\end{aligned}
$$

where $r=\left\{\begin{array}{ll}p, & \text { if } \operatorname{tp}(\sigma, \tau) \text { is even, } \\ q, & \text { if } \operatorname{tp}(\sigma, \tau) \text { is odd; }\end{array} \quad\right.$ and $T_{(\sigma, \tau)}^{\prime}=\emptyset$ if $(\sigma, \tau) \notin S^{\prime}$.

Then $P$ is $\sigma$-centered because

$$
P=\bigcup\left\{P_{\left\{T_{(\sigma, \tau)} \mid(\sigma, \tau) \in S\right\}} \mid\left\{T_{(\sigma, \tau)} \mid(\sigma, \tau) \in S\right\} \text { is as in the definition of } P\right\},
$$

where $P_{\left\{T_{(\sigma, \tau)} \mid(\sigma, \tau) \in S\right\}}:=\left\{\left(F,\left\{T_{(\sigma, \tau)} \mid(\sigma, \tau) \in S\right\}\right) \in P \mid F \subseteq \kappa\right.$ finite $\}$. There are only countable many sets $\left\{T_{(\sigma, \tau)} \mid(\sigma, \tau) \in S\right\}$ and obviously each set $P_{\left\{T_{(\sigma, \tau)} \mid(\sigma, \tau) \in S\right\}}$ is centered.

In the following we define suitable dense subsets of $P$ : For $\alpha<\kappa$ let

$$
D_{\alpha}:=\left\{\left(F,\left\{T_{(\sigma, \tau)} \mid(\sigma, \tau) \in S\right\}\right) \in P \mid \alpha \in F\right\} .
$$

Clearly, $D_{\alpha}$ is dense for every $\alpha<\kappa$.

We define

$$
E_{s, t, n}:=\left\{\left(F,\left\{T_{(\sigma, \tau)} \mid(\sigma, \tau) \in S\right\}\right) \mid(s, t) \in S \wedge \exists \mu \in T_{(s, t)}(\mu(|s|)>n)\right\}
$$

for $n \in \omega$ and $s, t \in{ }^{<\omega} \omega$ such that $\operatorname{tp}(s, t)$ is even and

$$
F_{s, t, m}:=\left\{\left(F,\left\{T_{(\sigma, \tau)} \mid(\sigma, \tau) \in S\right\}\right) \mid(s, t) \in S \wedge \exists \nu \in T_{(s, t)}(\nu(|t|)>m)\right\}
$$

for $m \in \omega$ and $s, t \in{ }^{<\omega} \omega$ such that $\operatorname{tp}(s, t)$ is odd.

Claim 1: The set $E_{s, t, n}$ is dense for every $n \in \omega$ and every $s, t \in{ }^{<\omega} \omega$ such that $\operatorname{tp}(s, t)$ is even and $F_{s, t, m}$ is dense for every $m \in \omega$ and every $s, t \in{ }^{<\omega} \omega$ such that $\operatorname{tp}(s, t)$ is odd.

Proof of claim 1: We only prove the even case, the odd case is similar. Suppose $n \in \omega$ and $s, t$ are in ${ }^{<\omega} \omega$ such that $\operatorname{tp}(s, t)$ even and $\left(F,\left\{T_{(\sigma, \tau)} \mid(\sigma, \tau) \in\right.\right.$ $S\}) \in P \backslash E_{s, t, n}$. Define $S^{\prime}:=S \cup\{(s, t)\}$ (maybe $\left.S=S^{\prime}\right)$. Let $\left(\alpha_{0}, \ldots, \alpha_{k-1}\right)$ be an ascending enumeration of $F$. Then we have $A_{\alpha_{0}} \geq^{*} \ldots \geq^{*} A_{\alpha_{k-1}}$ and this implies that the set $\bigcap_{i<k} A_{(s, t)}^{\alpha_{i}}$ is infinite. We have to care about property (f) in the definition of $P$ : Suppose there exists $\tau$ with $(s, \tau) \in S$ and $t \in T_{(s, \tau)}$ ( $\tau$ is unique); else we have nothing to do. Let $\left(t_{j}\right)_{j<N}$ be an enumeration of all $t^{\prime}$ with $t^{\prime} \in T_{(s, \tau)},\left(s, t^{\prime}\right) \in S$ and $t^{\prime}(|\tau|)<t(|\tau|)$ such that $t_{j}(|\tau|)<t_{j+1}(|\tau|)$ for every $j<N-1$. Then we have $A_{\left(s, t_{0}\right)}^{\alpha_{i}} \supseteq^{*} A_{\left(s, t_{1}\right)}^{\alpha_{i}} \supseteq^{*} \ldots \supseteq^{*} A_{(s, t)}^{\alpha_{i}}$ for every 
$i<k$ and therefore $\bigcap_{i<k} A_{(s, t)}^{\alpha_{i}} \subseteq^{*} \bigcap_{i<k} A_{\left(s, t_{N-1}\right)}^{\alpha_{i}} \subseteq^{*} \ldots \subseteq^{*} \bigcap_{i<k} A_{\left(s, t_{0}\right)}^{\alpha_{i}}$. So the set

$$
\bigcap_{i<k} A_{(s, t)}^{\alpha_{i}} \cap \bigcap_{i<k} A_{\left(s, t_{N-1}\right)}^{\alpha_{i}} \cap \ldots \cap \bigcap_{i<k} A_{\left(s, t_{0}\right)}^{\alpha_{i}}
$$

is infinite. Choose $\mu \in \bigcap_{i<k} A_{(s, t)}^{\alpha_{i}} \cap \bigcap_{i<k} A_{\left(s, t_{N-1}\right)}^{\alpha_{i}} \cap \ldots \cap \bigcap_{i<k} A_{\left(s, t_{0}\right)}^{\alpha_{i}}$ with $\mu(|s|)>n$ and $\mu \notin \pi_{1}(S)=\{s \mid \exists t((s, t) \in S)\}$. Define $T_{(s, t)}^{\prime}:=T_{(s, t)} \cup\{\mu\}$ $\left(T_{(s, t)}=\emptyset\right.$ if $\left.(s, t) \notin S\right), T_{\left(s, t_{j}\right)}^{\prime}:=T_{\left(s, t_{j}\right)} \cup\{\mu\}$ for every $j<N$ and $T_{(\sigma, \tau)}^{\prime}:=T_{(\sigma, \tau)}$ for every $(\sigma, \tau) \in S \backslash\left(\{(s, t)\} \cup\left\{\left(s, t_{j}\right) \mid j<N\right\}\right)$. We have $T_{\left(s, t^{\prime}\right)}^{\prime} \backslash T_{\left(s, t^{\prime}\right)}=\{\mu\} \subseteq$ $\operatorname{split}\left(p_{\left(s, t^{\prime}\right)}^{\left.\mathcal{A}^{\alpha_{i}}\right)}\right.$ for every $t^{\prime} \in\left\{t_{j} \mid j<N-1\right\} \cup\{t\}$, hence $\left(F,\left\{T_{(\sigma, \tau)}^{\prime} \mid(\sigma, \tau) \in\right.\right.$ $\left.S\} \cup\left\{T_{(s, t)}^{\prime}\right\}\right) \leq\left(F,\left\{T_{(\sigma, \tau)} \mid(\sigma, \tau) \in S\right\}\right)$ and $\left(F,\left\{T_{(\sigma, \tau)}^{\prime} \mid(\sigma, \tau) \in S\right\} \cup\left\{T_{(s, t)}^{\prime}\right\}\right)$ is in $D_{\alpha}$.

By MA( $\sigma$-centered $)$ let $G$ be a generic filter for the following dense sets: $E_{s, t, n}$ for $s, t \in{ }^{<\omega} \omega$ such that $\operatorname{tp}(s, t)$ is even and $n \in \omega, F_{s, t, m}$ for $s, t \in{ }^{<\omega} \omega$ such that $\operatorname{tp}(s, t)$ is odd and $m \in \omega$ and $D_{\alpha}$ for $\alpha<\kappa$. For $(s, t) \in I_{(p, q)}$ define

$$
B_{(s, t)}:=\left\{\rho \in \epsilon^{<\omega} \omega \mid \exists\left(F,\left\{T_{(\sigma, \tau)} \mid(\sigma, \tau) \in S\right\}\right) \in G\left((s, t) \in S \wedge \rho \in T_{(s, t)}\right)\right\} .
$$

Claim 2: $\mathcal{B}:=\left(B_{(s, t)}\right)_{(s, t) \in I_{(p, q)}}$ is a $\operatorname{good}(p, q)$-sequence.

Proof of claim 2: First suppose $\operatorname{tp}(s, t)$ is even and $\rho, \rho^{\prime} \in B_{(s, t)}$ with $\rho \neq \rho^{\prime}$. So there are $\left(F,\left\{T_{(\sigma, \tau)} \mid(\sigma, \tau) \in S\right\}\right)$ and $\left(F^{\prime},\left\{T_{(\sigma, \tau)}^{\prime} \mid(\sigma, \tau) \in S^{\prime}\right\}\right)$ in $G$ with $\rho \in T_{(s, t)}$ and $\rho^{\prime} \in T_{(s, t)}^{\prime}$, and there exists $\left(F^{\prime \prime},\left\{T_{(\sigma, \tau)}^{\prime \prime} \mid(\sigma, \tau) \in\right.\right.$ $\left.\left.S^{\prime \prime}\right\}\right) \leq\left(F,\left\{T_{(\sigma, \tau)} \mid(\sigma, \tau) \in S\right\}\right),\left(F^{\prime},\left\{T_{(\sigma, \tau)}^{\prime} \mid(\sigma, \tau) \in S^{\prime}\right\}\right)$ in $G$. So we have $T_{(s, t)}^{\prime \prime} \supseteq T_{(s, t)}, T_{(s, t)}^{\prime}$ and hence $\rho(|s|) \neq \rho^{\prime}(|s|)$. The case that $\operatorname{tp}(s, t)$ is odd is analogous, so (ii) of the definition of a good sequence (definition 2.1.1) holds.

For (i) we have to prove that $\left|B_{(s, t)}\right|$ is infinite. If $\operatorname{tp}(s, t)$ is even, for every $n \in \omega$ there exists $\left(F,\left\{T_{(\sigma, \tau)} \mid(\sigma, \tau) \in S\right\}\right) \in G \cap E_{s, t, n}$, hence $(s, t) \in S$ and there is a $\mu \in T_{(s, t)}$ with $\mu(|s|)>n$. If $\operatorname{tp}(s, t)$ is odd we can find for every $n \in \omega$ a $\nu \in T_{(s, t)}$ with $\nu(|t|)>n$.

(iii) and (iv) are easy to verify. For (v) suppose $\operatorname{tp}(s, t)$ is even and $\rho \in B_{(s, t)}$. Fix $\nu \in B_{(\rho, \tau)}$. Since $G \cap E_{s, t, n} \neq \emptyset$ for every $n \in \omega$, we get $\rho^{\prime} \in B_{(s, t)}$ such that $\rho^{\prime}(|s|)>\# \nu$, hence $\nu \notin B_{\left(\rho^{\prime}, t\right)}$, hence we have $B_{(\rho, t)} \supsetneqq B_{\left(\rho^{\prime}, t\right)}$. $\square($ claim 2)

Claim 3: $\mathcal{B} \leq^{*} \mathcal{A}^{\alpha}$ for every $\alpha<\kappa$.

Proof of claim 3: Suppose $\alpha<\kappa$ and fix $\left(F,\left\{T_{(\sigma, \tau)} \mid(\sigma, \tau) \in S\right\}\right) \in G \cap D_{\alpha}$.

Subclaim 1: For every $(s, t) \in I_{(p, q)} \backslash S$ we have $B_{(s, t)} \subseteq \operatorname{split}\left(r_{(s, t)}^{\mathcal{A}^{\alpha}}\right)$ where $r=p$ if $\operatorname{tp}(s, t)$ is even and $r=q$ if $\operatorname{tp}(s, t)$ is odd. 
Proof of subclaim 1: Suppose $(s, t) \in I_{(p, q)} \backslash S$ and $\operatorname{tp}(s, t)$ is even. Let $\mu \in B_{(s, t)}$, then there exists $\left(F^{\prime},\left\{T_{(\sigma, \tau)}^{\prime} \mid(\sigma, \tau) \in S^{\prime}\right\}\right) \in G$ with $(s, t) \in$ $S^{\prime}$ and $\mu \in T_{(s, t)}^{\prime}$. Choose $\left(F^{\prime \prime},\left\{T_{(\sigma, \tau)}^{\prime \prime} \mid(\sigma, \tau) \in S^{\prime \prime}\right\}\right) \leq\left(F,\left\{T_{(\sigma, \tau)} \mid(\sigma, \tau) \in\right.\right.$ $S\}),\left(F^{\prime},\left\{T_{(\sigma, \tau)}^{\prime} \mid(\sigma, \tau) \in S^{\prime}\right\}\right)$ in $G$. Then we have $(s, t) \in S^{\prime \prime}, \mu \in T_{(s, t)}^{\prime \prime}$, $(s, t) \notin S$ and $\alpha \in F$ and these imply $\mu \in T_{(s, t)}^{\prime \prime} \backslash T_{(s, t)}=T_{(s, t)}^{\prime \prime} \subseteq \operatorname{split}\left(p_{(s, t)}^{\mathcal{A}^{\alpha}}\right)$, as desired. The odd case is once more analogous.

Subclaim 2: For the finitely many $(s, t) \in S$ we have $B_{(s, t)} \subseteq^{*} \operatorname{split}\left(r_{(s, t)}^{\mathcal{A}^{\alpha}}\right)$ for $r=p$ if $\operatorname{tp}(s, t)$ is even and $r=q$ if $\operatorname{tp}(s, t)$ is odd.

Proof of subclaim 2: Suppose $(s, t) \in S$ and $\operatorname{tp}(s, t)$ is even. We prove that we have $B_{(s, t)} \backslash T_{(s, t)} \subseteq \operatorname{split}\left(p_{(s, t)}^{\mathcal{A}^{\alpha}}\right)$. Suppose $\mu \in B_{(s, t)} \backslash T_{(s, t)}$, so there exists $\left(F^{\prime},\left\{T_{(\sigma, \tau)}^{\prime} \mid(\sigma, \tau) \in S^{\prime}\right\}\right) \in G$ with $(s, t) \in S^{\prime}$ and $\mu \in T_{(s, t)}^{\prime}$. Choose $\left(F^{\prime \prime},\left\{T_{(\sigma, \tau)}^{\prime \prime} \mid(\sigma, \tau) \in S^{\prime \prime}\right\}\right) \leq\left(F,\left\{T_{(\sigma, \tau)} \mid(\sigma, \tau) \in S\right\}\right),\left(F^{\prime},\left\{T_{(\sigma, \tau)}^{\prime} \mid(\sigma, \tau) \in S^{\prime}\right\}\right)$ in $G$. Then we have $\mu \in T_{(s, t)}^{\prime \prime}, \mu \notin T_{(s, t)}$ and $\alpha \in F$ and these imply $\mu \in$ $T_{(s, t)}^{\prime \prime} \backslash T_{(s, t)} \subseteq \operatorname{split}\left(p_{(s, t)}^{\mathcal{A}^{\alpha}}\right)$. The odd case is analogous.

$\square($ subclaim 2) Subclaim 1 and 2 together easily imply $\mathcal{B} \leq{ }^{*} \mathcal{A}^{\alpha}$.

$\square($ claim 3)

\subsection{The proof of the maintheorem}

Now we are able to prove the main theorem of this chapter, but we first write down a generalized version:

Theorem 2.2.1 $\mathrm{MA}_{\kappa}(\sigma$-centered $)$ implies $\operatorname{add}\left(\mathcal{J}\left(\mathbb{M}^{2}\right)\right)>\kappa$.

Proof of theorem 2.2.1 and therefore of theorem 2.0.5: Suppose $\mathrm{MA}_{\kappa}(\sigma-$ centered) is true and $X_{\alpha} \in J\left(\mathbb{M}^{2}\right)$ for $\alpha<\kappa$ and $(p, q) \in \mathbb{M}^{2}$. We search for a pair $\left(p^{\prime}, q^{\prime}\right) \leq(p, q)$ in $\mathbb{M}^{2}$ such that $\left(\left[p^{\prime}\right] \times\left[q^{\prime}\right]\right) \cap \bigcup_{\alpha<\kappa} X_{\alpha}=\emptyset$. Define by induction a $\leq^{*}$-descending sequence $\left(\mathcal{A}^{\alpha}\right)_{\alpha \leq \kappa}$ of $\operatorname{good}(p, q)$-sequences: For $\alpha=0$ define $\mathcal{A}^{0}$ to be the good sequence for $(p, q)$ defined in remark 2.1.2. Suppose $\mathcal{A}^{\beta}$ for $\beta<\alpha$ is already defined and $\alpha<\kappa$ is a limit ordinal. Choose by lemma 2.1.8 (we use $\mathrm{MA}_{\kappa}\left(\sigma\right.$-centered) here) a good $(p, q)$-sequence $\mathcal{A}^{\alpha}$ with $\mathcal{A}^{\alpha} \leq^{*} \mathcal{A}^{\beta}$ for every $\beta<\alpha$.

For the successor step, suppose $\mathcal{A}^{\alpha}$ is defined. Choose a good $(p, q)$-sequence $\mathcal{A}^{\alpha+1} \leq^{*} \mathcal{A}^{\alpha}$ with $\left(\left[p_{(\sigma, \tau)}^{\mathcal{A}^{\alpha+1}}\right] \times\left[q_{(\sigma, \tau)}^{\mathcal{A}^{\alpha+1}}\right]\right) \cap X_{\alpha}=\emptyset$ for every $(\sigma, \tau) \in I_{(p, q)}$ by lemma 2.1.7.

Notice that the pair $\left(p_{(\mathrm{st}(p), \mathrm{st}(q))}^{\mathcal{A}^{\kappa}}, q_{(\mathrm{st}(p), \mathrm{st}(q))}^{\mathcal{A}^{\kappa}}\right)$ of Miller trees does not have the desired property, because for $\alpha<\kappa$ we only have $\mathcal{A}^{\kappa} \leq^{*} \mathcal{A}^{\alpha+1}$, hence there 
could exist a pair $(f, g) \in\left[p_{(\mathrm{st}(p), \mathrm{st}(q))}^{\mathcal{A}^{\kappa}}\right] \times\left[q_{(\mathrm{st}(p), \mathrm{st}(q))}^{\mathcal{A}^{\kappa}}\right]$ with $(f, g) \notin\left[p_{(\mathrm{st}(p), \mathrm{st}(q))}^{\mathcal{A}^{\alpha+1}}\right] \times$ $\left[q_{(\mathrm{st}(p), \mathrm{st}(q))}^{\mathcal{A}^{\alpha+1}}\right]$, therefore we do not know whether $(f, g)$ is in $X$ or not. We will define a forcing $Q$ to get $\left(p^{\prime}, q^{\prime}\right) \leq\left(p_{(\operatorname{st}(p), \mathrm{st}(q))}^{\mathcal{A}^{\kappa}}, q_{(\mathrm{st}(p), \mathrm{st}(q))}^{\mathcal{A}^{\kappa}}\right)$ such that for every $(f, g) \in\left[p^{\prime}\right] \times\left[q^{\prime}\right]$ and every $\alpha<\kappa$ we have $(f, g) \in\left[p_{(f|n, g| m)}^{\mathcal{A}^{\alpha+1}}\right] \times\left[q_{(f|n, g| m)}^{\mathcal{A}^{\alpha+1}}\right]$ for suitable $n, m \in \omega$. In this situation the choice of $\mathcal{A}^{\alpha+1}$ guarantees $(f, g) \notin X$.

Define $Q$ in the following way: $Q$ is the set of all $(u, v, X)$ with

(a') $X \subseteq \kappa$ is finite;

(b') $u: m \rightarrow \operatorname{split}\left(p_{(\operatorname{st}(p), \operatorname{st}(q))}^{\mathcal{A}^{\kappa}}\right)$ and $v: m^{\prime} \rightarrow \operatorname{split}\left(q_{(\mathrm{st}(p), \operatorname{st}(q))}^{\mathcal{A}^{\kappa}}\right), m, m^{\prime} \in \omega$ with $m=m^{\prime}$ or $m=m^{\prime}+1$

(c') the downward closures $U$ and $V$ of $\operatorname{ran}(u)$ and $\operatorname{ran}(v)$ are finite subtrees of $p_{(\operatorname{st}(p), \mathrm{st}(q))}^{\mathcal{A}^{\kappa}}, q_{(\mathrm{st}(p), \mathrm{st}(q))}^{\mathcal{A}^{\kappa}}$, respectively, with $\operatorname{split}(U) \subseteq \operatorname{ran}(u)$ and $\operatorname{split}(V) \subseteq \operatorname{ran}(v)$;

(d') the functions $\Phi:(\operatorname{ran}(u), \leq) \rightarrow(T\lceil m, \leq)$ defined by $u(k) \longmapsto T(k)$ and $\Psi:(\operatorname{ran}(v), \leq) \rightarrow\left(T\left\lceil m^{\prime}, \leq\right)\right.$ defined by $v(k) \longmapsto T(k)$ are both isomorphisms;

(e') if $u(j)$ is the immediate predecessor in the tree sense of $u(k+1)$ in $\operatorname{ran}(u)$, then we have $u(k+1)(|u(j)|)>\max (\{\# u(i) \mid i \leq k\} \cup\{\# v(i) \mid i \leq k\})$ and $|u(k+1)|>u(k+1)(|u(j)|)$,

if $v(j)$ is the immediate predecessor in the tree sense of $v(k+1)$ in $\operatorname{ran}(v)$, then we have $v(k+1)(|v(j)|)>\max (\{\# u(i) \mid i \leq k+1\} \cup\{\# v(i) \mid i \leq k\})$ and $|v(k+1)|>v(k+1)(|v(j)|)$.

Define $(u, v, X) \leq\left(u^{\prime}, v^{\prime}, X^{\prime}\right): \Leftrightarrow$

$$
\begin{aligned}
& u \supseteq u^{\prime}, v \supseteq v^{\prime}, X \supseteq X^{\prime} \text { and } \\
& \text { for every } \alpha \in X^{\prime} \text { and every }(\sigma, \tau) \in(\operatorname{ran}(u) \times \operatorname{ran}(v)) \backslash \\
& \left(\operatorname{ran}\left(u^{\prime}\right) \times \operatorname{ran}\left(v^{\prime}\right)\right) \text { we have }(\sigma, \tau) \in p_{(\sigma|n, \tau| m)}^{\mathcal{A}^{\alpha}} \times q_{(\sigma|n, \tau| m)}^{\mathcal{A}^{\alpha}} \\
& \text { for every } n, m \in \omega \text { such that }\left(\sigma \left\lceiln, t\lceil m) \in I_{(p, q)} .\right.\right.
\end{aligned}
$$

Q is $\sigma$-centered, because $Q=\bigcup\left\{Q_{(u, v)} \mid(u, v)\right.$ is as in the definition of $\left.Q\right\}$, where $Q_{(u, v)}:=\{(u, v, X) \in Q \mid X \subseteq \kappa$ is finite $\}$ is obviously centered and there are only countable many $(u, v)$.

In the following, we will define suitable dense subsets of $Q$ : For $\alpha<\kappa$ define

$$
D_{\alpha}:=\{(u, v, X) \in Q \mid \alpha \in X\} .
$$


Clearly, $D_{\alpha}$ is dense for every $\alpha<\kappa$.

We define

$$
\begin{aligned}
E_{s, t, n}:=\{(u, v, X) \in Q \mid(s, t) \in & \operatorname{ran}(u) \times \operatorname{ran}(v) \wedge \\
& \exists \mu \in \operatorname{ran}(u)(s \varsubsetneqq \mu \wedge \mu(|s|)>n)\}
\end{aligned}
$$

for $(s, t) \in I_{(p, q)}$ with $\operatorname{tp}(s, t)$ even and $n \in \omega$ and

$$
\begin{aligned}
F_{s, t, m}:=\{(u, v, X) \in Q \mid(s, t) \in & \operatorname{ran}(u) \times \operatorname{ran}(v) \wedge \\
& \quad \exists \in \operatorname{ran}(v)(t \varsubsetneqq \nu \wedge \nu(|t|)>m)\}
\end{aligned}
$$

for $(s, t) \in I_{(p, q)}$ with $\operatorname{tp}(s, t)$ odd and $m \in \omega$. For $n \in \omega$ and $(s, t) \in I_{(p, q)}$ with $\operatorname{tp}(s, t)$ even the set $E_{s, t, n}$ is dense below every $(\tilde{u}, \tilde{v}, \tilde{X})$ with $(s, t) \in$ $\operatorname{ran}(\tilde{u}) \times \operatorname{ran}(\tilde{v})$. And for $m \in \omega$ and $(s, t) \in I_{(p, q)}$ with $\operatorname{tp}(s, t)$ odd the set $F_{s, t, m}$ is dense below every $(\tilde{u}, \tilde{v}, \tilde{X})$ with $(s, t) \in \operatorname{ran}(\tilde{u}) \times \operatorname{ran}(\tilde{v})$. The proof of this equals the proof of claim 1 in lemma 2.1.8 and is skipped. Letting

$$
\begin{aligned}
& \tilde{E}_{s, t, n}:=\left\{(u, v, X) \in Q \mid \exists\left(u^{\prime}, v^{\prime}, X^{\prime}\right) \in E_{s, t, n}\left((u, v, X) \leq\left(u^{\prime}, v^{\prime}, X^{\prime}\right)\right)\right\} \\
& \cup\left\{(u, v, X) \in Q \mid(u, v, X) \text { is incompatible with every element of } E_{s, t, n}\right\}
\end{aligned}
$$

and

$$
\begin{aligned}
& \tilde{F}_{s, t, m}:=\left\{(u, v, X) \in Q \mid \exists\left(u^{\prime}, v^{\prime}, X^{\prime}\right) \in F_{s, t, m}\left((u, v, X) \leq\left(u^{\prime}, v^{\prime}, X^{\prime}\right)\right)\right\} \\
& \cup\left\{(u, v, X) \in Q \mid(u, v, X) \text { is incompatible with every element of } F_{s, t, m}\right\}
\end{aligned}
$$

we get dense subsets of $Q$. By $\mathrm{MA}_{\kappa}(\sigma$-centered $)$ let $G$ be a generic filter for the dense sets $D_{\alpha}$ for $\alpha<\kappa, \tilde{E}_{s, t, n}$ for $(s, t) \in I_{(p, q)}$ with $\operatorname{tp}(s, t)$ even and $n \in \omega$ and $\tilde{F}_{s, t, m}$ with $(s, t) \in I_{(p, q)}$ with $\operatorname{tp}(s, t)$ odd and $m \in \omega$. Define $p^{\prime}$ and $q^{\prime}$ to be the superperfect trees determined by

$$
\operatorname{split}\left(p^{\prime}\right)=\bigcup\{\operatorname{ran}(u) \mid \exists v \exists X((u, v, X) \in G)\}
$$

and by

$$
\operatorname{split}\left(q^{\prime}\right)=\bigcup\{\operatorname{ran}(v) \mid \exists u \exists X((u, v, X) \in G)\} .
$$

Claim 1: $\left(p^{\prime}, q^{\prime}\right) \in \mathbb{M}^{2}$.

Proof of claim 1: Suppose $(s, t) \in p^{\prime} \times q^{\prime}$. Then there exists $(u, v, X) \in G$ and $s^{\prime} \in \operatorname{ran}(u)$ and $t^{\prime} \in \operatorname{ran}(v)$ with $s^{\prime} \supseteq s, t^{\prime} \supseteq t,\left(s^{\prime}, t^{\prime}\right) \in \operatorname{ran}(u) \times \operatorname{ran}(v)$ and $\operatorname{tp}\left(s^{\prime}, t^{\prime}\right)$ exists. If $\operatorname{tp}\left(s^{\prime}, t^{\prime}\right)$ is even, we have $G \cap E_{s^{\prime}, t^{\prime}, n} \neq \emptyset$ for every $n \in \omega$, therefore $s^{\prime}$ is an infinite splitnode above $s$, hence $p^{\prime} \in \mathbb{M}$. If $\operatorname{tp}\left(s^{\prime}, t^{\prime}\right)$ is odd we have $G \cap F_{s^{\prime}, t^{\prime}, m} \neq \emptyset$ for some $m \in \omega$ and it is possible to choose extensions $s^{\prime \prime}$ of $s$ and $t^{\prime \prime}$ of $t^{\prime}$ with $\operatorname{tp}\left(s^{\prime \prime}, t^{\prime \prime}\right)$ is even. Again, we have $G \cap E_{s^{\prime \prime}, t^{\prime \prime}, n} \neq \emptyset$ for every $n \in \omega$ and therefore $s^{\prime \prime}$ is an infinite splitnode above $s$. Analogously, we 
get $q^{\prime} \in \mathbb{M}$.

$\square($ claim 1)

Obviously, we have $\left(p^{\prime}, q^{\prime}\right) \leq(p, q)$.

Claim 2: $\left(\left[p^{\prime}\right] \times\left[q^{\prime}\right]\right) \cap \bigcup_{\alpha<\kappa} X_{\alpha}=\emptyset$.

Proof of claim 2: It suffices to prove $\left(\left[p^{\prime}\right] \times\left[q^{\prime}\right]\right) \cap X_{\alpha}=\emptyset$ for every $\alpha<\kappa$. Fix $\alpha<\kappa$ and $(u, v, X) \in G \cap D_{\alpha+1}$, so $\alpha+1 \in X$ and let $U$ and $V$ be the downward closures of $\operatorname{ran}(u), \operatorname{ran}(v)$, respectively.

Subclaim: For every $(s, t) \in\left(p^{\prime} \backslash U\right) \times\left(q^{\prime} \backslash V\right)$ we have $(s, t) \in p_{(s|n, t| m)}^{\mathcal{A}^{\alpha+1}} \times q_{(s|n, t| m)}^{\mathcal{A}^{\alpha+1}}$ for every $n, m \in \omega$ such that $\left(s\left\lceil n, t\lceil m) \in I_{(p, q)}\right.\right.$.

Proof of the subclaim: Suppose $(s, t) \in\left(p^{\prime} \backslash U\right) \times\left(q^{\prime} \backslash V\right)$ and choose $\left(s^{\prime}, t^{\prime}\right) \geq$ $(s, t)$ with $\left(s^{\prime}, t^{\prime}\right) \in\left(\left(p^{\prime} \backslash U\right) \times\left(q^{\prime} \backslash V\right)\right) \cap\left(\operatorname{split}\left(p_{(\operatorname{st}(p), \operatorname{st}(q))}^{\mathcal{A}^{\kappa}}\right) \times \operatorname{split}\left(q_{(\operatorname{st}(p), \operatorname{st}(q))}^{\mathcal{A}^{\kappa}}\right)\right)$. There are $\left(u^{\prime}, v^{\prime}, X^{\prime}\right),\left(u^{\prime \prime}, v^{\prime \prime}, X^{\prime \prime}\right) \in G$ such that $s^{\prime} \in \operatorname{ran}\left(u^{\prime}\right)$ and $t^{\prime} \in \operatorname{ran}\left(v^{\prime \prime}\right)$. Choose $(\bar{u}, \bar{v}, \bar{X}) \leq\left(u^{\prime}, v^{\prime}, X^{\prime}\right),\left(u^{\prime \prime}, v^{\prime \prime}, X^{\prime \prime}\right)$ in $G$ and then $(\tilde{u}, \tilde{v}, \tilde{X}) \leq(\bar{u}, \bar{v}, \bar{X})$, $(u, v, X)$ in $G$, as well. Then we have $s^{\prime} \in \operatorname{ran}(\tilde{u}) \backslash \operatorname{ran}(u), t^{\prime} \in \operatorname{ran}(\tilde{v}) \backslash \operatorname{ran}(v)$ and $\alpha+1 \in X$ and these imply $\left(s^{\prime}, t^{\prime}\right) \in\left(p_{\left(s^{\prime}\left|n, t^{\prime}\right| m\right)}^{\mathcal{A}^{\alpha+1}} \times q_{\left(s^{\prime}\left|n, t^{\prime}\right| m\right)}^{\mathcal{A}^{\alpha+1}}\right)$ for every $n, m \in \omega$ such that $\left(s^{\prime}\left|n, t^{\prime}\right| m\right) \in I_{(p, q)}$. Therefore, $(s, t)$ is in $p_{\left(s^{\prime} \uparrow n, t^{\prime} \uparrow m\right)}^{\mathcal{A}^{\alpha+1}} \times q_{\left(s^{\prime}\left|n, t^{\prime}\right| m\right)}^{\mathcal{A}^{\alpha+1}}$, as well.

$\square$ (subclaim)

Now we are able to prove claim 2: For $(f, g) \in\left[p^{\prime}\right] \times\left[q^{\prime}\right]$ we get $(f, g) \in$ $\left[p_{(f\lceil n, g \mid m)}^{\mathcal{A}^{\alpha+1}}\right] \times\left[q_{(f\lceil n, g \mid m)}^{\mathcal{A}^{\alpha+1}}\right]$ for suitable $n, m \in \omega$ and we have chosen $\mathcal{A}^{\alpha+1}$ in such a way that $\left(\left[p_{(f\lceil n, g \mid m)}^{\mathcal{A}^{\alpha+1}}\right] \times\left[q_{(f\lceil n, g \mid m)}^{\mathcal{A}^{\alpha+1}}\right]\right) \cap X_{\alpha}=\emptyset$ hold.

$\square($ claim 2)

$\square($ theorem 2.2.1 and 2.0.5)

Since $\mathrm{MA}_{\kappa}(\sigma$-centered $)$ is true for every $\kappa<\mathrm{p}$, where $\mathrm{p}$ is the least cardinality of a filter base on $\left([\omega]^{\omega}, \subseteq^{*}\right)$ without any lower bound, theorem 2.2.1 implies the following:

Corollary 2.2.2 $\operatorname{add}\left(\mathcal{J}\left(\mathbb{M}^{2}\right)\right) \geq \mathrm{p}$.

Notice also that we have $\operatorname{cov}\left(\mathcal{J}\left(\mathbb{M}^{2}\right)\right) \leq \mathrm{d}$ like in the one-dimensional case, where $d$ is the dominating number, i.e. the least cardinality of a dominating family in $\left({ }^{\omega} \omega, \leq^{*}\right)$. For this, let $\left(f_{\alpha}\right)_{\alpha<\mathrm{d}}$ be a dominating family in ${ }^{\omega} \omega$ and define $X_{\alpha}:=\left\{(f, g) \in{ }^{\omega} \omega \times{ }^{\omega} \omega \mid f, g \leq{ }^{*} f_{\alpha}\right\}$. Then it is easy to see that $\bigcup_{\alpha<\mathrm{d}} X_{\alpha}={ }^{\omega} \omega \times{ }^{\omega} \omega$ and clearly, each $X_{\alpha}$ is in $\mathcal{J}\left(\mathbb{M}^{2}\right)$. Summerized, we have

$$
\omega_{1} \leq \mathrm{p} \leq \operatorname{add}\left(\mathcal{J}\left(\mathbb{M}^{2}\right)\right) \leq \operatorname{cov}\left(\mathcal{J}\left(\mathbb{M}^{2}\right)\right) \leq \mathrm{d} \leq 2^{\omega}
$$


Let us remark, that in the model, built for proving the maintheorem 1.1.2 in the previous chapter, we have $\operatorname{add}\left(\mathcal{J}\left(\mathbb{M}^{2}\right)\right)>\operatorname{cov}(\mathcal{M}) \geq \mathrm{t} \geq \mathrm{p}$, where $\mathrm{t}$ is the tower number, that is the least cardinality of a decreasing chain in $\left([\omega]^{\omega}, \subseteq^{*}\right)$ without any lower bound. The last inequality is trivial, the second one is a result of Piotrowski and Szymański [PiSz].

\subsection{MA( $\sigma$-centered $)$ implies Martin's Axiom for $\mathbb{M}^{2}$}

The following definitions are due to Veličković [Ve].

Definition 2.3.1 For a forcing $P$ and a dense set $D \subseteq P$ we say that a subordering $Q \subseteq P$ captures the density of $D$ if $D \cap Q$ is dense in $Q$.

If $\mathcal{D}$ is a family of dense sets of $P$, we say that $Q$ captures the density of $\mathcal{D}$ if $Q$ captures the density of each $D \in \mathcal{D}$.

Definition 2.3.2 For a forcing $P$ and a class $\mathcal{Z}$ of forcings let $\mathcal{Z}(P)$ be the following statement:

Whenever $\mathcal{D}$ is a family of at most $2^{\omega}$ dense subsets of $P$ and $p \in P$, then there exists a $Q \in \mathcal{Z}$ such that $p \in Q \subseteq P$ and $Q$ captures the density of $\mathcal{D}$.

Definition 2.3.3 For a forcing $P$ define $\operatorname{MA}(P)$ to be the statement:

For any collection of less than $2^{\omega}$ dense subsets of $P$ there exists a filter on $P$ intersecting all of them.

For a class $\mathcal{Z}$ of forcings $\mathrm{MA}(\mathcal{Z})$ means that $\mathrm{MA}(P)$ is true for every $P$ in $\mathcal{Z}$.

Thus, the usual MA is $\mathrm{MA}(c c c)$, where $c c c$ denotes the class of all forcings satisfying the countable chain condition.

It is easy to prove the following:

Fact 2.3.4 For a class $\mathcal{Z}$ of forcings and a single forcing $P, \operatorname{MA}(\mathcal{Z})$ and $\mathcal{Z}(P)$ together imply $\mathrm{MA}(P)$.

We want to prove the following result:

Theorem 2.3.5 $\mathrm{MA}(\sigma$-centered $)$ implies $\mathrm{MA}\left(\mathbb{M}^{2}\right)$.

Since MA implies $\mathrm{MA}(\sigma$-centered $)$ we get that $\mathrm{MA}$ implies $\mathrm{MA}\left(\mathbb{M}^{2}\right)$.

Because of fact 2.3.4, the following theorem implies theorem 2.3.5. 
Theorem 2.3.6 $\mathrm{MA}(\sigma$-centered $)$ implies $\sigma$-centered $\left(\mathbb{M}^{2}\right)$.

For the proof we need the following definition and lemmata:

Definition 2.3.7 Suppose $(p, q) \in \mathbb{M}^{2}$. For a good $(p, q)$-sequence $\mathcal{A}$ define

$$
\begin{aligned}
\mathbb{M}_{\mathcal{A}}^{2}:=\{(u, v) & \in \mathbb{M}^{2} \mid(\operatorname{st}(u), \operatorname{st}(v)) \in I_{(p, q)} \wedge \\
\exists \mathcal{A}^{\prime} \approx \mathcal{A} \forall(\sigma, \tau) \in I_{(p, q)} \cap(\operatorname{split}(u) \times \operatorname{split}(v)) & \left.\left(\left(p_{(\sigma, \tau)}^{\mathcal{A}^{\prime}}, q_{(\sigma, \tau)}^{\mathcal{A}^{\prime}}\right) \leq(u, v)\right)\right\} .
\end{aligned}
$$

Lemma 2.3.8 Suppose $(p, q) \in \mathbb{M}^{2}$ and suppose $\mathcal{A}$ and $\mathcal{B}$ are good $(p, q)$ sequences. Then the following hold:

(a) $\mathbb{M}_{\mathcal{A}}^{2}$ is $\sigma$-centered.

(b) $\mathcal{A} \approx \mathcal{B}$ implies $\mathbb{M}_{\mathcal{A}}^{2}=\mathbb{M}_{\mathcal{B}}^{2}$.

(c) $\mathcal{A} \leq^{*} \mathcal{B}$ implies $\mathbb{M}_{\mathcal{A}}^{2} \supseteq \mathbb{M}_{\mathcal{B}}^{2}$.

Proof: For (a): For $(s, t) \in I_{(p, q)}$ define

$$
M_{(s, t)}^{\mathcal{A}}:=\left\{(u, v) \in \mathbb{M}_{\mathcal{A}}^{2} \mid \operatorname{st}(u)=s \wedge \operatorname{st}(v)=t\right\} .
$$

Then we have $\mathbb{M}_{\mathcal{A}}^{2}=\bigcup\left\{M_{(s, t)}^{\mathcal{A}} \mid(s, t) \in I_{(p, q)}\right\}$ and since $I_{(p, q)}$ is countable, it suffices to prove that each $M_{(s, t)}^{\mathcal{A}}$ is centered. Fix $(s, t)$ and assume $(u, v),\left(u^{\prime}, v^{\prime}\right) \in$ $M_{(s, t)}^{\mathcal{A}}$. Then there are good sequences $\mathcal{B}$ and $\mathcal{B}^{\prime}$ witnessing this. Suppose $\operatorname{tp}(s, t)$ is even, the odd case is similar. It is easy to find a set $C_{(s, t)}$ such that $C_{(s, t)}={ }^{*} A_{(s, t)}, C_{(s, t)}={ }^{*} B_{(s, t)}$ and

$$
\forall \sigma \in C_{(s, t)} \forall \sigma^{\prime} \supseteq \sigma \forall \tau \supseteq t\left(\operatorname{tp}\left(\sigma^{\prime}, \tau\right) \text { exists } \Rightarrow B_{\left(\sigma^{\prime}, \tau\right)}=B_{\left(\sigma^{\prime}, \tau\right)}^{\prime}\right) .
$$

Define $C_{(\sigma, \tau)}:=B_{(\sigma, \tau)}$ for $(\sigma, \tau) \neq(s, t)$. Then $\mathcal{C}=\left(C_{(\sigma, \tau)}\right)_{(\sigma, \tau) \in I_{(p, q)}}$ is a good sequence with $\mathcal{B} \approx \mathcal{C} \approx \mathcal{B}^{\prime}$ and $\mathcal{C} \approx \mathcal{A}$. And $\left(p_{(s, t)}^{\mathcal{C}}, q_{(s, t)}^{\mathcal{C}}\right)$ is an element of $M_{(s, t)}^{\mathcal{A}}$ smaller then $(u, v)$ and $\left(u^{\prime}, v^{\prime}\right)$.

(b) is clear.

For (c): Suppose we have $\mathcal{A} \leq^{*} \mathcal{B}$, so there exists a good $(p, q)$-sequence $\mathcal{A}^{\prime}$ with $\mathcal{A} \approx \mathcal{A}^{\prime} \leq \mathcal{B}$, and suppose $(u, v) \in \mathbb{M}_{\mathcal{B}}^{2}$, witnessed by $\mathcal{B}^{\prime}$. By lemma 2.1.5 there exists a good $(p, q)$-sequence $\mathcal{A}^{\prime \prime}$ such that $\mathcal{A}^{\prime} \approx \mathcal{A}^{\prime \prime} \leq \mathcal{B}^{\prime}$. Then for every $\forall(\sigma, \tau) \in I_{(p, q)} \cap(\operatorname{split}(u) \times \operatorname{split}(v))$ we have $\left(p_{(\sigma, \tau)}^{\mathcal{A}^{\prime \prime}}, q_{(\sigma, \tau)}^{\mathcal{A}^{\prime \prime}}\right) \leq\left(p_{(\sigma, \tau)}^{\mathcal{B}^{\prime}}, q_{(\sigma, \tau)}^{\mathcal{B}^{\prime}}\right) \leq$ $(u, v)$, so $\mathcal{A}^{\prime \prime}$ witnesses that $(u, v) \in \mathbb{M}_{\mathcal{A}}^{2}$.

Lemma 2.3.9 Suppose $(p, q) \in \mathbb{M}^{2}$ and $\mathcal{A}$ is good for $(p, q),(u, v) \in \mathbb{M}^{2}, D \subseteq$ $\mathbb{M}^{2}$ is dense and there exists a good $(p, q)$-sequence $\mathcal{B} \leq^{*} \mathcal{A}$ with $(u, v) \in \mathbb{M}_{\mathcal{B}}^{2}$. Then there exist $\mathcal{C} \leq^{*} \mathcal{A}$ and a pair $\left(u^{\prime}, v^{\prime}\right) \leq(u, v)$ such that $(u, v) \in \mathbb{M}_{\mathcal{C}}^{2}$ and $\left(u^{\prime}, v^{\prime}\right) \in D \cap \mathbb{M}_{\mathcal{C}}^{2}$. If $D$ is $\leq^{0}$-dense, we can choose $\left(u^{\prime}, v^{\prime}\right) \leq^{0}(u, v)$. 
Proof: Suppose $\mathcal{B} \leq^{*} \mathcal{A}$ and $(u, v) \in \mathbb{M}_{\mathcal{B}}^{2}$, witnessed by $\mathcal{B}^{\prime}$. Since $D$ is dense we find $\left(u^{\prime}, v^{\prime}\right) \leq\left(p_{(\operatorname{st}(u), \operatorname{st}(v))}^{\mathcal{B}^{\prime}}, q_{(\mathrm{st}(u), \mathrm{st}(v))}^{\mathcal{B}^{\prime}}\right) \leq(u, v)$ with $\left(u^{\prime}, v^{\prime}\right) \in D$ (If $D$ is $\leq^{0}$ dense, we choose $\left(u^{\prime}, v^{\prime}\right)$ such that $\operatorname{st}\left(u^{\prime}\right)=\operatorname{st}(u)$ and $\operatorname{st}\left(v^{\prime}\right)=\operatorname{st}(v)$.). We define $\mathcal{C}$ in the following way: If $\operatorname{tp}(\sigma, \tau)$ is even and $(\sigma, \tau) \in I_{(p, q)} \cap(\operatorname{split}(u) \times \operatorname{split}(v))$ let $C_{(\sigma, \tau)}$ be a subset of $\operatorname{Succ}_{u^{\prime}}(\sigma) \cap\{\rho \mid \rho(|\sigma|)>\# \sigma\}$ such that for every $\rho \in$ $C_{(\sigma, \tau)}$ we have $|\rho|>\rho(|\sigma|), \min \{\rho(|\sigma|),|\rho|\}>\# \tau$ and $\rho(|\sigma|)>\# \rho^{\prime}$ for every $\rho^{\prime} \in$ $C_{(\sigma, \tau)}$ with $\rho^{\prime}(|\sigma|)<\rho(|\sigma|)$. Analogously for $(\sigma, \tau) \in I_{(p, q)} \cap(\operatorname{split}(u) \times \operatorname{split}(v))$ and $\operatorname{tp}(\sigma, \tau)$ is odd. For $(\sigma, \tau) \in I_{(p, q)} \backslash(\operatorname{split}(u) \times \operatorname{split}(v))$ let $C_{(\sigma, \tau)}:=B_{(\sigma, \tau)}^{\prime}$. If $\operatorname{tp}(\sigma, \tau)$ is even we have $C_{(\sigma, \tau)} \subseteq \operatorname{split}\left(p_{(\sigma, \tau)}^{\mathcal{B}^{\prime}}\right)$, if $\operatorname{tp}(\sigma, \tau)$ is odd we have $C_{(\sigma, \tau)} \subseteq \operatorname{split}\left(q_{(\sigma, \tau)}^{\mathcal{B}^{\prime}}\right)$, so $\mathcal{C} \leq \mathcal{B}^{\prime} \leq^{*} \mathcal{A}$ and therefore $\mathcal{C} \leq^{*} \mathcal{A}$, and we have $(u, v) \in \mathbb{M}_{\mathcal{B}^{\prime}}^{2}=\mathbb{M}_{\mathcal{B}}^{2} \subseteq \mathbb{M}_{\mathcal{C}}^{2}$ by lemma 2.3 .8 (c). It is easy to prove that $\left(u^{\prime}, v^{\prime}\right)$ is in $\mathbb{M}_{\mathcal{C}}^{2}$.

Now we are able to prove theorem 2.3.6:

Proof of theorem 2.3.6: Suppose MA( $\sigma$-centered $)$ is true. Suppose also $\mathcal{D}=\left\{D_{\alpha} \mid \alpha<2^{\omega}\right\}$ is a family of dense subsets of $\mathbb{M}^{2}$ and $(p, q) \in \mathbb{M}^{2}$. Let

$$
\left\{\left(\left(p_{\alpha}, q_{\alpha}\right), D_{\alpha}\right) \mid \alpha<2^{\omega}\right\}
$$

be an enumeration of $\mathbb{M}^{2} \times \mathcal{D}$ with $\left(p_{0}, q_{0}\right)=(p, q)$. We have to find a $\sigma$-centered forcing $Q$ such that $(p, q) \in Q \subseteq \mathbb{M}^{2}$ and $Q$ captures the density of $\mathcal{D}$.

Define a $\leq^{*}$-descending sequence $\left(\mathcal{A}^{\alpha}\right)_{\alpha<2^{\omega}}$ of good $(p, q)$-sequences as follows: For $\alpha=0$ define $\mathcal{A}^{0}$ to be the good sequence for $\left(p_{0}, q_{0}\right)=(p, q)$ defined in remark 2.1.2. Then clearly $(p, q) \in \mathbb{M}_{\mathcal{A}^{0}}^{2}$.

If $\left(\mathcal{A}^{\beta}\right)_{\beta<\alpha}$ is already defined and $\alpha$ is a limit ordinal, we choose by $\operatorname{MA}(\sigma$ centered) and lemma 2.1 .8 a good $(p, q)$-sequence $\mathcal{A}^{\alpha}$ such that $\mathcal{A}^{\alpha} \leq^{*} \mathcal{A}^{\beta}$ for every $\beta<\alpha$.

For the successor step suppose $\mathcal{A}^{\alpha}$ is already defined. If there is no good $(p, q)$ sequence $\mathcal{A} \leq^{*} \mathcal{A}^{\alpha}$ with $\left(p_{\alpha}, q_{\alpha}\right) \in \mathbb{M}_{\mathcal{A}}^{2}$, let $\mathcal{A}^{\alpha+1}:=\mathcal{A}^{\alpha}$.

If there exists $\mathcal{A} \leq^{*} \mathcal{A}^{\alpha}$ with $\left(p_{\alpha}, q_{\alpha}\right) \in \mathbb{M}_{\mathcal{A}}^{2}$, lemma 2.3 .9 gives us a good $(p, q)$ sequence $\mathcal{B} \leq^{*} \mathcal{A}^{\alpha}$ such that $\left(p_{\alpha}, q_{\alpha}\right) \in \mathbb{M}_{\mathcal{B}}^{2}$ and there is a pair $\left(p_{\alpha}^{\prime}, q_{\alpha}^{\prime}\right) \leq\left(p_{\alpha}, q_{\alpha}\right)$ with $\left(p_{\alpha}^{\prime}, q_{\alpha}^{\prime}\right) \in D_{\alpha} \cap \mathbb{M}_{\mathcal{B}}^{2}$. Let $\mathcal{A}^{\alpha+1}:=\mathcal{B}$.

In the end, define

$$
Q:=\bigcup_{\alpha<2^{\omega}} \mathbb{M}_{\mathcal{A}^{\alpha}}^{2}
$$

Clearly, we have $Q \subseteq \mathbb{M}^{2}$.

Claim 1: $Q$ is $\sigma$-centered. 
Proof of claim 1: For $(\sigma, \tau) \in I_{(p, q)}$ define $Q_{(\sigma, \tau)}:=\{(u, v) \in Q \mid \operatorname{st}(u)=$ $\sigma \wedge \operatorname{st}(v)=\tau\}$, then we have $Q=\bigcup\left\{Q_{(\sigma, \tau)} \mid(\sigma, \tau) \in I_{(p, q)}\right\} . I_{(p, q)}$ is countable and like in the proof of lemma 2.3.8 (a) using that $\left(A^{\alpha}\right)_{\alpha<2^{\omega}}$ is a $\leq^{*}$-descending chain, we see that every $Q_{(\sigma, \tau)}$ is centered.

$\square($ claim 1)

Claim 2: $Q$ captures the density of $\mathcal{D}$.

Proof of claim 2: Suppose $D \in \mathcal{D}$ and $(\bar{p}, \bar{q}) \in Q$, so there exists $\alpha<2^{\omega}$ such that $((\bar{p}, \bar{q}), D)=\left(\left(p_{\alpha}, q_{\alpha}\right), D_{\alpha}\right)$. We have to find $\left(p^{\prime}, q^{\prime}\right) \leq(\bar{p}, \bar{q})$ such that $\left(p^{\prime}, q^{\prime}\right) \in D \cap Q$.

Since $(\bar{p}, \bar{q}) \in Q$ we have $(\bar{p}, \bar{q}) \in \mathbb{M}_{\mathcal{A}^{\beta}}^{2}$ for some $\beta$, by lemma 2.3 .8 we have $(\bar{p}, \bar{q}) \in \mathbb{M}_{\mathcal{A} \gamma}^{2}$ for every $\gamma \geq \beta$. Hence, at step $\alpha$ in the construction of the sequence $\left(\mathcal{A}^{\alpha}\right)_{\alpha<2^{\omega}}$ arises the second case. There we have got $\left(p_{\alpha}^{\prime}, q_{\alpha}^{\prime}\right) \leq(\bar{p}, \bar{q})$ such that $\left(p_{\alpha}^{\prime}, q_{\alpha}^{\prime}\right) \in D \cap \mathbb{M}_{\mathcal{A}^{\alpha+1}}^{2} \subseteq D \cap Q$. Let $\left(p^{\prime}, q^{\prime}\right):=\left(p_{\alpha}^{\prime}, q_{\alpha}^{\prime}\right)$ and we are done.

$\square($ claim 2)

Corollary 2.3.10 MA( $\sigma$-centered $)$ implies that $\mathbb{M}^{2}$ does not collaps cardinals.

Proof: Since $\mathbb{M}^{2}$ clearly satisfies the $\left(2^{\omega}\right)^{+}$-chain-condition, it could only collapse cardinals $\leq 2^{\omega}$. Suppose there exist cardinals $\kappa$ and $\lambda$ such that $\kappa<\lambda \leq$ $2^{\omega}$ and there is a $\mathbb{M}^{2}$-name $\tau$ with

$$
\Vdash_{\mathbb{M}^{2}} \tau: \kappa \rightarrow \lambda \text { is surjective. }
$$

Define for each $\alpha<\lambda$

$$
D_{\alpha}:=\left\{(p, q) \in \mathbb{M}^{2} \mid \exists \xi<\kappa\left((p, q) \Vdash_{\mathbb{M}^{2}} \tau(\xi)=\alpha\right)\right\} .
$$

Then $D_{\alpha}$ is open and dense for every $\alpha<\lambda$. As MA( $\sigma$-centered $)$ is true, we have $\sigma$-centered $\left(\mathbb{M}^{2}\right)$ and since $\lambda \leq 2^{\omega}$ there exists a forcing $P \subseteq \mathbb{M}^{2}$ such that each $D_{\alpha} \cap P$ is dense in $P$ and $P$ is $\sigma$-centered. But then $P$ collapses $\kappa$ to $\lambda$, this is a contradiction to the fact that $P$ fulfills the countable chain condition. 


\section{Chapter 3}

\section{Infinite maximal antichains in $(\mathfrak{P}(\omega) / \text { fin })^{n^{*}}$}

\subsection{Definitions and combinatorics}

In the sequel, we always identify the elements of $\mathfrak{P}(\omega)$ /fin with their representatives in $[\omega]^{\omega}$. By $(\mathfrak{P}(\omega) / \text { fin })^{n^{*}}$, for $n^{*}>0$ in $\omega$, we mean the full product carrying the coordinatewise ordering. Hence elements of $(\mathfrak{P}(\omega) / \text { fin })^{n^{*}}$ are $n^{*}$ tupels of subsets of $\omega$, elements of $(\mathfrak{P}(\omega) / \text { fin } \backslash\{0\})^{n^{*}}$ are $n^{*}$-tupels of infinite subsets of $\omega$, or we can view the elements as functions from $n^{*}$ to $\mathfrak{P}(\omega) /$ fin, $\mathfrak{P}(\omega) /$ fin $\backslash\{0\}$, respectively. For $\bar{a} \in(\mathfrak{P}(\omega) / \text { fin } \backslash\{0\})^{n^{*}}$ and $F \subseteq n^{*}$ we write $\bar{a}\lceil F$ for the restricted function $\bar{a}: F \rightarrow \mathfrak{P}(\omega) /$ fin $\backslash\{0\}$. If $F$ have just one element $i$, we write $a_{i}$ instead of $\bar{a}\left\lceil\{i\}\right.$, so $\bar{a}=\left(a_{i}\right)_{i<n^{*}}$.

Two elements $\bar{a}, \bar{b} \in(\mathfrak{P}(\omega) / \text { fin } \backslash\{0\})^{n^{*}}$ are called compatible if there exists an element $\bar{c} \in(\mathfrak{P}(\omega) / \text { fin } \backslash\{0\})^{n^{*}}$ with $\bar{c} \leq \bar{a}$ and $\bar{c} \leq \bar{b}$, i.e. for $\bar{a}=\left(a_{i}\right)_{i<n^{*}}$ and $\bar{b}=\left(b_{i}\right)_{i<n^{*}}$ that $a_{i} \cap b_{i}$ is infinite for every $i<n^{*}$. As usual, if $\bar{a}$ and $\bar{b}$ are not compatible, they are called incompatible. A subset $A \subseteq(\mathfrak{P}(\omega) / \mathrm{fin} \backslash\{0\})^{n^{*}}$ of pairwise incompatible elements is called an antichain of $(\mathfrak{P}(\omega) / \mathrm{fin})^{n^{*}}$.

Here, we are interested in infinite maximal antichains of $(\mathfrak{P}(\omega) / \text { fin })^{n^{*}}$. Since $(\mathfrak{P}(\omega) / \mathrm{fin})^{n^{*}}$ can be densely embedded in the complete Boolean algebra of the regular open subsets of $(\mathfrak{P}(\omega) / \mathrm{fin})^{n^{*}}$ (see [Ko], for example), people often use the terminology of Boolean algebras and call maximal antichains in $(\mathfrak{P}(\omega) / \text { fin })^{n^{*}}$ partitions.

Of course there are finite analytical maximal antichains of $(\mathfrak{P}(\omega) / \text { fin })^{n^{*}}$ for every $n^{*} \in \omega \backslash\{0\}$ : choose $a \in[\omega]^{\omega}$ with $\omega \backslash a \in[\omega]^{\omega}$, too, then $\left\{\bar{a}=\left(a_{i}\right)_{i<n^{*}} \mid a_{i} \in\right.$ $\{a, \omega \backslash a\}$ for every $\left.i<n^{*}\right\}$ is a maximal antichain in $(\mathfrak{P}(\omega) / \mathrm{fin})^{n^{*}}$.

Our goal is to proof, analogously to Mathias' one-dimensional result, the following theorem: 
Theorem 3.1.1 Fix $n^{*} \in \omega \backslash\{0\}$.

There are no analytical infinite maximal antichains in $(\mathfrak{P}(\omega) / \mathrm{fin})^{n^{*}}$.

Recall that a filter on $\omega$ is called Ramsey if for every descending sequence $\left(X_{n}\right)_{n \in \omega}$ with $X_{n} \in U$ for every $n \in \omega$ there exists $\left\{x_{n} \mid n \in \omega\right\} \in U$ such that $x_{n+1} \in X_{x_{n}}$ for every $n \in \omega$. In this case, we say that $\left\{x_{n} \mid n \in \omega\right\}$ diagonalizes the sequence $\left(X_{n}\right)_{n \in \omega}$. If $f: \omega \rightarrow \omega$ is an enumerating function for the set $\left\{x_{n} \mid n \in \omega\right\}$, hence $f(n+1) \in X_{f(n)}$ for every $n \in \omega$, we say that $f$ diagonalizes $\left(X_{n}\right)_{n \in \omega}$.

The Rudin-Keisler ordering $\leq_{R K}$ is defined by the following: For two ultrafilters $U$ and $U^{\prime}$ we have $U \leq_{R K} U^{\prime}$ if there exists a function $h: \omega \rightarrow \omega$ such that $U=\left\{x \subseteq \omega \mid h^{-1}[x] \in U^{\prime}\right\}$. Two ultrafilters $U, U^{\prime}$ are called Rudin-Keislerequivalent if and only if $U \leq_{R K} U^{\prime}$ and $U^{\prime} \leq_{R K} U$. Equivalently (see for example [Je]), $U$ and $U^{\prime}$ are Rudin-Keisler-equivalent if there exists a bijection $h: \omega \rightarrow \omega$ such that $U=\left\{h[x] \mid x \in U^{\prime}\right\}$.

It is well known that Martin's Axiom implies the existence of a Ramsey ultrafilter and the continuums hypothesis $(\mathrm{CH})$ implies the existence as well (this is for example a consequence of lemma 3.3.1). And also a $\mathfrak{P}(\omega) /$ fin-generic filter over $V$ is a Ramsey ultrafilter. Kunen got, starting with a model of $\mathrm{CH}$ and adding $\aleph_{2}$ random reals, a model in which there are no Ramsey ultrafilters (see for example [Je]).

For $n^{*} \in \omega \backslash\{0\}$ and an infinite maximal antichain $\mathcal{A} \subseteq(\mathfrak{P}(\omega) / \text { fin } \backslash\{0\})^{n^{*}}$ define

$$
\begin{aligned}
& \mathcal{J}(\mathcal{A}):=\left\{\bar{x} \in(\mathfrak{P}(\omega) / \text { fin } \backslash\{0\})^{n^{*}} \mid\right. \exists k \in \omega \exists \bar{a}_{0}, \ldots, \bar{a}_{k} \in \mathcal{A} \\
&\left.\left(\bar{x} \backslash\left(\bar{a}_{0} \cup \ldots \cup \bar{a}_{k}\right) \notin(\mathfrak{P}(\omega) / \text { fin } \backslash\{0\})^{n^{*}}\right)\right\}
\end{aligned}
$$

and

$$
\begin{aligned}
\mathcal{J}(\mathcal{A})^{+} & :=(\mathfrak{P}(\omega) / \text { fin } \backslash\{0\})^{n^{*}} \backslash \mathcal{J}(\mathcal{A}) \\
& =\left\{\bar{x} \in(\mathfrak{P}(\omega) / \text { fin } \backslash\{0\})^{n^{*}} \mid \exists^{\infty} \bar{a} \in \mathcal{A}(\bar{a} \text { and } \bar{x} \text { are compatible })\right\}
\end{aligned}
$$

Here, $\exists^{\infty}$ means "there are infinitely many". For $\bar{x} \in(\mathfrak{P}(\omega) / \text { fin } \backslash\{0\})^{n^{*}}$ define

$$
\mathcal{A}\lceil\bar{x}:=\{\bar{a} \in \mathcal{A} \mid \bar{a} \text { and } \bar{x} \text { are compatible }\} \text {, }
$$

the set of all elements of $\mathcal{A}$ compatible with $\bar{x}$.

We will use the following lemma of [Sp4]:

Lemma 3.1.2 [lemma 1.1 of [Sp4]]

Suppose $n^{*} \in \omega \backslash\{0\}$ and $\mathcal{A} \subseteq(\mathfrak{P}(\omega) / \mathrm{fin})^{n^{*}}$ is an infinite maximal antichain. If $F \subseteq n^{*}$ is (with respect to $\subseteq$ ) maximal such that there exists $\mathcal{F} \in[\mathcal{A}]^{\omega}$ with the property that $\{\bar{a}|F| \bar{a} \in \mathcal{F}\}$ has the finite intersection property in $(\mathfrak{P}(\omega) / \text { fin } \backslash\{0\})^{n^{*}}$, then $|\mathcal{F}|=n^{*}-1$. 
Here, the finite intersection property in $(\mathfrak{P}(\omega) / \text { fin } \backslash\{0\})^{n^{*}}$ means, that for every finite subset there is a lower bound in $(\mathfrak{P}(\omega) / \text { fin } \backslash\{0\})^{n^{*}}$. In other words, every finite coordinatewise intersection of elements of $\mathcal{F}$ is in every coordinate infinite. Of course, this lemma also holds for the restriction $\mathcal{A}\lceil\bar{x}$ for every $\bar{x}=\left(x_{0}, \ldots, x_{n^{*}-1}\right) \in(\mathfrak{P}(\omega) / \text { fin })^{n^{*}}$.

Corollary 3.1.3 Suppose $n^{*}$ and $\mathcal{A}$ are as in lemma 3.1.2 and $\bar{x}$ is an element of $(\mathfrak{P}(\omega) / \text { fin } \backslash\{0\})^{n^{*}}$. Then there exist $\mathcal{F} \in\left[\mathcal{A}\lceil\bar{x}]^{\omega}\right.$ and $i^{*} \in n^{*}$ such that for every $i \in n^{*} \backslash\left\{i^{*}\right\}$ there exists $b_{i} \in[\omega]^{\omega}$ with $b_{i} \subseteq^{*} a_{i}$ for every $\bar{a} \in \mathcal{F}$. Clearly, the set $\left\{a_{i^{*}} \mid \bar{a} \in \mathcal{F}\right\}$ must be an antichain.

Lemma 3.1.4 Suppose $n^{*} \in \omega \backslash\{0\}$ and $\mathcal{A} \subseteq(\mathfrak{P}(\omega) / \mathrm{fin})^{n^{*}}$ is an infinite maximal antichain. Suppose also $\bar{b}=\left(b_{0}, \ldots, b_{n^{*}-1}\right) \in \mathcal{J}(\mathcal{A})^{+}$and $h: \omega \rightarrow \omega$ is a bijection.

Then for every pair $k, l \in n^{*}$ with $k<l$ there exists $b_{k}^{\prime} \subseteq b_{k}$ and $b_{l}^{\prime} \subseteq b_{l}$ such that we still have $\left(b_{0}, \ldots, b_{k}^{\prime}, \ldots, b_{l}^{\prime}, \ldots, b_{n^{*}-1}\right) \in \mathcal{J}(\mathcal{A})^{+}$and additionally $h\left[b_{k}^{\prime}\right] \cap b_{l}^{\prime}=\emptyset$ or $h\left[b_{l}^{\prime}\right] \cap b_{k}^{\prime}=\emptyset$ holds.

Proof: So suppose $\bar{b} \in \mathcal{J}(\mathcal{A})^{+}, h: \omega \rightarrow \omega$ is bijective and $k<l<n^{*}$ are given. By corollary 3.1.3 there exist $i^{*}<n^{*}$ and $\mathcal{F} \in\left[\mathcal{A}\lceil\bar{b}]^{\omega}\right.$ such that for every $i \in n^{*} \backslash\left\{i^{*}\right\}$ there exists $b_{i}^{*} \in[\omega]^{\omega}$ with $b_{i}^{*} \subseteq^{*} c_{i}$ for every $\bar{c} \in \mathcal{F}$. Let $\left(\bar{c}^{n}\right)_{n \in \omega}$ be an enumeration of $\mathcal{F}$. Notice that the set $\left\{c_{i^{*}}^{n} \mid n \in \omega\right\}$ consists of pairwise almost disjoint subsets of $\omega$, since $\mathcal{F}$ is countable without loss of generality we have $c_{i^{*}}^{n} \cap c_{i^{*}}^{m}=\emptyset$ for $n \neq m$.

First case: $k, l \in n^{*} \backslash\left\{i^{*}\right\}$.

Consider the set $\left(b_{l}^{*} \cap b_{l}\right) \backslash h\left[b_{k}^{*} \cap b_{k}\right]$. If this set is infinite, let $b_{l}^{\prime}:=\left(b_{l}^{*} \cap b_{l}\right) \backslash$ $h\left[b_{k}^{*} \cap b_{k}\right]$ and $b_{k}^{\prime}:=b_{k}^{*} \cap b_{k}$. Then clearly we have $h\left[b_{k}^{\prime}\right] \cap b_{l}^{\prime}=\emptyset$ and because of $b_{k}^{\prime} \subseteq b_{k}^{*}, b_{l}^{\prime} \subseteq b_{l}^{*}$ and the choice of $b_{k}^{*}$ and $b_{l}^{*}$ we have $\left(b_{0}, \ldots, b_{k}^{\prime}, \ldots, b_{l}^{\prime}, \ldots, b_{n^{*}-1}^{\prime}\right)$ is compatible with every $\bar{c} \in \mathcal{F}$, hence it is an element of $\mathcal{J}(\mathcal{A})^{+}$.

If $\left(b_{l}^{*} \cap b_{l}\right) \backslash h\left[b_{k}^{*} \cap b_{k}\right]$ is finite, divide the infinite set $b_{l}^{*} \cap b_{l} \cap h\left[b_{k}^{*} \cap b_{k}\right]$ in two infinite pieces $b^{\prime} \dot{\cup} b^{\prime \prime}$ and let $b_{l}^{\prime}:=b^{\prime}$ and $b_{k}^{\prime}:=h^{-1}\left[b^{\prime \prime}\right]$. Again, we have $\left(b_{0}, \ldots, b_{k}^{\prime}, \ldots, b_{l}^{\prime}, \ldots, b_{n^{*}-1}^{\prime}\right) \in \mathcal{J}(\mathcal{A})^{+}$.

Second case: $l=i^{*}$.

If there is an infinite subsequence $\left(c_{i^{*}}^{n_{m}}\right)_{m \in \omega}$ of $\left(c_{i^{*}}^{n}\right)_{n \in \omega}$ such that $c_{i^{*}}^{n_{m}} \cap h\left[b_{k} \cap\right.$ $\left.b_{k}^{*}\right] \cap b_{l}$ is infinite for every $m \in \omega$ then let

$$
\begin{aligned}
b_{k}^{\prime} & :=h^{-1}\left[\bigcup_{m \in \omega} c_{i^{*}}^{n_{2 m}}\right] \cap\left(b_{k} \cap b_{k}^{*}\right) \quad \text { and } \\
b_{l}^{\prime} & :=\bigcup_{m \in \omega} c_{i^{*}}^{n_{2 m+1}} \cap b_{l} .
\end{aligned}
$$

If there are only finitely many $c_{i^{*}}^{n_{0}}, \ldots, c_{i^{*}}^{n_{u}}$ such that $c_{i^{*}}^{n_{m}} \cap h\left[b_{k} \cap b_{k}^{*}\right] \cap b_{l}$ is infinite for every $m \leq u$ then let

$$
\begin{aligned}
b_{k}^{\prime} & :=b_{k} \cap b_{k}^{*} \quad \text { and } \\
b_{l}^{\prime}:= & \left(\bigcup\left\{c_{i^{*}}^{n} \mid n \in \omega \backslash\left\{n_{0}, \ldots, n_{u}\right\}\right\} \cap b_{l}\right) \backslash h\left[b_{k} \cap b_{k}^{*}\right] .
\end{aligned}
$$


Easily, in both subcases $b_{k}^{\prime}$ and $b_{l}^{\prime}$ are as claimed.

Third case: $k=i^{*}$.

This case is analogous to the second one, we just have to change the roles of $k$ and $l$.

\subsection{A finite-dimensional version of Mathias forcing}

Now we introduce an $n^{*}$-dimensional version of Mathias forcing:

Definition 3.2.1 [ShSp2] Fix $n^{*} \in \omega \backslash\{0\}$. Let

$$
Q:=\left\{(s, S) \in[\omega]^{<\omega} \times[\omega]^{\omega} \mid \max (s)<\min (S) \vee s=\emptyset\right\} .
$$

For defining the ordering on $Q$ we need the following: let $\left(l_{j}\right)_{j<|s|}$ be the increasing enumeration of $s \in[\omega]^{<\omega}$ and define $s_{i}:=\left\{l_{j} \mid j \equiv i\left(\bmod n^{*}\right)\right\}$ for $i<n^{*}$ and let $\left(k_{j}\right)_{j<\omega}$ be the increasing enumeration of $S \in[\omega]^{\omega}$ and define $S_{i}:=\left\{k_{j} \mid j \equiv i\left(\bmod n^{*}\right)\right\}$ for $i<n^{*}$. Then we define

$$
\begin{aligned}
(s, S) \leq(t, T): \Leftrightarrow \quad & s \cap(\max t+1)=t \text { or } t=\emptyset, \\
& s_{i} \backslash t_{i} \subseteq T_{i} \text { for every } i<n^{*} \text { and } \\
& S_{i} \subseteq T_{i} \text { for every } i<n^{*} .
\end{aligned}
$$

We write $(s, S) \leq^{0}(t, T)$ if $(s, S) \leq(t, T)$ and additionally $s=t$.

Notice that $(s, S),(t, T) \in Q$ are compatible if and only if

$$
\left(\forall i<n^{*}\left(s_{i} \subseteq t_{i} \wedge t_{i} \backslash s_{i} \subseteq S_{i}\right)\right) \vee\left(\forall i<n^{*}\left(t_{i} \subseteq s_{i} \wedge s_{i} \backslash t_{i} \subseteq T_{i}\right)\right)
$$

and for every $i<n^{*}$ we have $\left|S_{i} \cap T_{i}\right|=\omega$.

As for the classical Mathias forcing we have the following relationship between $Q$-reals and generic filters: If $G$ is $Q$-generic over some model $M$ define $x:=$ $\bigcup\{s \mid \exists S((s, S) \in G)\}$ and $x_{i}:=\left\{k_{j} \mid j \equiv i\left(\bmod n^{*}\right)\right\}$, where $\left(k_{i}\right)_{i<\omega}$ is the increasing enumeration of $x$ (notice that we have $x_{i}=\bigcup\left\{s_{i} \mid \exists S^{\prime}\left(\left(s^{\prime}, S^{\prime}\right) \in\right.\right.$ $\left.\left.\left.G \wedge s_{i}^{\prime}=s_{i}\right)\right\}\right)$. Then $G=\left\{(s, S) \in Q^{M} \mid \forall i<n^{*}\left(s_{i} \subseteq x_{i} \subseteq S_{i} \cup s_{i}\right)\right\}$. The proof of this fact equals the classical proof. Hence a set $x$ is a $Q$-real if the set $G_{x}:=\left\{(s, S) \in Q^{M} \mid \forall i<n^{*}\left(s_{i} \subseteq x_{i} \subseteq S_{i} \cup s_{i}\right)\right\}$ is a $Q$-generic filter over $M$, where $s_{i}$ is defined as above.

Suppose $U_{0}, \ldots, U_{n^{*}-1}$ are ultrafilters on $\omega$. Then we define the subordering $Q\left(U_{0}, \ldots, U_{n^{*}-1}\right)$ of $Q$ like follows: in $Q\left(U_{0}, \ldots, U_{n^{*}-1}\right)$ are only those pairs $(s, S) \in Q$ with $S_{i} \in U_{i}$ for every $i<n^{*}$.

In the following we list some of the results of the investigation of $Q$ by Shelah and Spinas [ShSp2] which we will need later. All this results generalize well known facts about the one-dimensional Mathias forcing (see [Ma], for example). Fix $n^{*} \in \omega \backslash\{0\}$. 
Fact 3.2.2 [ShSp2] The forcing $Q$ is equivalent to the forcing $(\mathfrak{P}(\omega) / \mathrm{fin})^{n^{*}} *$ $Q\left(\dot{G}_{0}, \ldots, \dot{G}_{n^{*}-1}\right)$, where $\left(\dot{G}_{0}, \ldots, \dot{G}_{n^{*}-1}\right)$ is the canonical name for the generic object added by the forcing $(\mathfrak{P}(\omega) / \mathrm{fin})^{n^{*}}$ consisting of pairwise not Rudin-Keisler-equivalent Ramsey ultrafilters.

Shelah and Spinas introduced a helpful game:

Definition 3.2.3 Let $U_{0}, \ldots, U_{n^{*}-1}$ be ultrafilters on $\omega$. Then the game $\Gamma\left(U_{0}, \ldots, U_{n^{*}-1}\right)$ for two players $I$ and $I I$ is defined as follows: player $I$ chooses in the $m$ th-move $\left(A_{0}^{m}, \ldots, A_{n^{*}-1}^{m}\right) \in U_{0} \times \ldots \times U_{n^{*}-1}$ and player $I I$ responds playing $k_{m} \in A_{m \bmod n^{*}}^{m}$. Player $I I$ wins the game if and only if for every $i<n^{*}$ the set $\left\{k_{n} \mid n \equiv i\left(\bmod n^{*}\right)\right\}$ is an element of $U_{i}$.

Fact 3.2.4 [ShSp2] Suppose $U_{0}, \ldots, U_{n^{*}-1}$ are pairwise not Rudin-Keisler-equivalent Ramsey ultrafilters. Then player $I$ does not have a winning strategy in the game $\Gamma\left(U_{0}, \ldots, U_{n^{*}-1}\right)$.

Here, it is easy to see that it is necessary that the ultrafilters are pairwise not Rudin-Keisler-equivalent.

Fact 3.2.5 [ShSp2] Let $U_{0}, \ldots, U_{n^{*}-1}$ are pairwise not Rudin-Keisler-equivalent Ramsey ultrafilters on $\omega$. Suppose $S \in[\omega]^{\omega}$ such that for every $i<n^{*}$ and for every $X \in U_{i}$ we have $S_{i} \subseteq^{*} X$. Then $S$ is $Q\left(U_{0}, \ldots, U_{n^{*}-1}\right)$-generic over $V$.

As a corollary we get:

Fact 3.2.6 [ShSp2] Let $U_{0}, \ldots, U_{n^{*}-1}$ are pairwise not Rudin-Keisler-equivalent Ramsey ultrafilters. If $S \in[\omega]^{\omega}$ is $Q\left(U_{0}, \ldots, U_{n^{*}-1}\right)$-generic over $V$ and $T \in[\omega]^{\omega}$ with $T_{i} \subseteq S_{i}$ for every $i<n^{*}$, then $T$ is $Q\left(U_{0}, \ldots, U_{n^{*}-1}\right)$-generic over $V$ as well.

Fact 3.2.7 [ShSp2] If $U_{0}, \ldots, U_{n^{*}-1}$ are pairwise not Rudin-Keisler-equivalent Ramsey ultrafilters then the forcing $Q\left(U_{0}, \ldots, U_{n^{*}-1}\right)$ has the pure decision property.

This means that for a given $Q\left(U_{0}, \ldots, U_{n^{*}-1}\right)$-name $\Theta$ such that $\Vdash_{Q\left(U_{0}, \ldots, U_{n^{*}-1}\right)} \Theta \in\{0,1\}$ and given $(s, S) \in Q\left(U_{0}, \ldots, U_{n^{*}-1}\right)$, there exist $(t, T) \in Q\left(U_{0}, \ldots, U_{n^{*}-1}\right)$ and $\varepsilon \in\{0,1\}$ such that $(t, T) \leq^{0}(s, S)$ and $(t, T) \Vdash_{Q\left(U_{0}, \ldots, U_{n^{*}-1}\right)} \Theta=\varepsilon$. 


\subsection{Finding Ramsey ultrafilters}

Suppose $\mathcal{A}$ is an infinite maximal antichain in $\mathfrak{P}(\omega) /$ fin. Mathias [Ma] proved that $\mathrm{CH}$ implies that there exists a Ramsey ultrafilter $U$ such that $U \subseteq \mathcal{J}(\mathcal{A})^{+}$. For the proof of Theorem 3.1.1 we need the following $n^{*}$-dimensional generalization:

Lemma 3.3.1 (CH) Suppose $n^{*} \in \omega \backslash\{0\}$ and $\mathcal{A}$ is an infinite maximal antichain in $(\mathfrak{P}(\omega) / \mathrm{fin})^{n^{*}}$. Then there exist pairwise not Rudin-Keisler-equivalent Ramsey ultrafilters $U_{0}, \ldots, U_{n^{*}-1}$ such that $U_{0} \times \ldots \times U_{n^{*}-1} \subseteq \mathcal{J}(\mathcal{A})^{+}$.

Proof: Assume $\mathrm{CH}$ and fix the following enumerations: Let $\left(x_{\alpha}\right)_{\alpha<\omega_{1}}$ enumerate $[\omega]^{\omega} ;\left(h_{\alpha}\right)_{\alpha<\omega_{1}}$ enumerate all bijections from $\omega$ to $\omega$ and let $\left({ }^{\alpha} \bar{X}\right)_{\alpha \in \operatorname{Lim}\left(\omega_{1}\right)}$ enumerate all $n^{*}$-tupels of decreasing sequences (of length $\omega$ ) in $[\omega]^{\omega}$, such that every ${ }^{\alpha} \bar{X}$ occurs cofinal often.

We want to construct sequences $\left(a_{0}^{\alpha}\right)_{\alpha<\omega_{1}}, \ldots,\left(a_{n^{*}-1}^{\alpha}\right)_{\alpha<\omega_{1}}$ in $[\omega]^{\omega}$ by induction such that we get $U_{i}$ as the filter generated by $\left\{a_{i}^{\alpha} \mid \alpha<\omega_{1}\right\}$ for $i<n^{*}$.

For beginning the induction choose $\left(a_{0}^{0}, \ldots, a_{n^{*}-1}^{0}\right) \in \mathcal{J}(\mathcal{A})^{+}$arbitrarily.

Suppose $\left(a_{0}^{\nu}\right)_{\nu<\alpha}, \ldots,\left(a_{n^{*}-1}^{\nu}\right)_{\nu<\alpha}$ are chosen such that $\left\{a_{i}^{\beta} \mid \beta<\nu\right\}$ generates a filter called $F_{i}^{\nu}$ for every $i<n^{*}$ and every $\nu<\alpha$ and we have $F_{0}^{\nu} \times \ldots \times F_{n^{*}-1}^{\nu} \subseteq$ $\mathcal{J}(\mathcal{A})^{+}$for every $\nu<\alpha$.

First, suppose that $\alpha$ is a successor ordinal, say $\alpha=\beta+1$. Consider the infinite subset $x_{\beta}$ of $\omega$. Let

$$
{ }^{0} b_{i}:=a_{i}^{\beta} \cap x_{\beta} \text { and }{ }^{1} b_{i}:=a_{i}^{\beta} \backslash x_{\beta}
$$

for $i<n^{*}$. Because of $\left(a_{0}^{\beta}, \ldots, a_{n^{*}-1}^{\beta}\right) \in \mathcal{J}(\mathcal{A})^{+}$there exists at least one sequence $\left(\varepsilon_{0}, \ldots, \varepsilon_{n^{*}-1}\right) \in\{0,1\}^{n^{*}}$ such that $\left({ }^{\varepsilon_{0}} b_{0}, \ldots,{ }^{\varepsilon_{n^{*}-1}} b_{n^{*}-1}\right) \in \mathcal{J}(\mathcal{A})^{+}$. Now consider the function $h_{\beta}$. By applying lemma 3.1.4 finitely many times we get the following: For every $i<n^{*}$ there is an infinite $a_{i} \subseteq{ }^{\varepsilon_{i}} b_{i}$ such that $\left(a_{0}, \ldots, a_{n^{*}-1}\right) \in \mathcal{J}(\mathcal{A})^{+}$and for every pair $k, l<n^{*}$ with $k \neq l$ we have $h_{\beta}\left[a_{k}\right] \cap a_{l}=\emptyset$ or $h_{\beta}\left[a_{l}\right] \cap a_{k}=\emptyset$. Let $a_{i}^{\alpha}:=a_{i}$ for every $i<n^{*}$.

Second, suppose that $\alpha$ is a limit ordinal. Consider the tupel ${ }^{\alpha} \bar{X}=$ $\left(\left({ }^{\alpha} X_{0}^{n}\right)_{n \in \omega}, \ldots,\left({ }^{\alpha} X_{n^{*}-1}^{n}\right)_{n \in \omega}\right)$ of decreasing sequences in $[\omega]^{\omega}$.

First case: $\Pi_{i<n^{*}}\left\{{ }^{\alpha} X_{i}^{n} \mid n \in \omega\right\} \subseteq \Pi_{i<n^{*}} \bigcup_{\nu<\alpha} F_{i}^{\nu}$. We want to construct for every $i<n^{*}$ strictly increasing functions $f_{i}: \omega \rightarrow \omega$ such that $f_{i}(n+1) \in$ ${ }^{\alpha} X_{i}^{f_{i}(n)}$ for every $i<n^{*}$ and every $n \in \omega,\left(f_{0}[\omega], \ldots, f_{n^{*}-1}[\omega]\right) \in \mathcal{J}(\mathcal{A})^{+}$and $\Pi_{i<n^{*}}\left(\bigcup_{\alpha<\nu} F_{i}^{\nu} \cup\left\{f_{i}[\omega]\right\}\right)$ has the finite intersection property in $\mathcal{J}(\mathcal{A})^{+}$.

Fix for every $i<n^{*}$ an enumeration $\left(a_{i}^{\prime n}\right)_{n \in \omega}$ of $\left\{a_{i}^{\nu} \mid \nu<\alpha\right\}$.

Choose $g_{i}^{0}(0) \in{ }^{\alpha} X_{i}^{0} \cap a_{i}^{\prime 0}$ for every $i<n^{*}$. If $g_{0}^{0}(k), \ldots, g_{n^{*}-1}^{0}(k)$ are already chosen for some $k \in \omega$, take for every $i<n^{*}$

$$
g_{i}^{0}(k+1) \in{ }^{\alpha} X_{i}^{0} \cap \ldots \cap{ }^{\alpha} X_{i}^{g_{i}^{0}(k)} \cap a_{i}^{\prime 0} \cap \ldots \cap a_{i}^{\prime k+1}
$$


with $g_{i}^{0}(k+1)>g_{i}^{0}(k)$ (notice that in this first case the set ${ }^{\alpha} X_{i}^{0} \cap . . \cap^{\alpha} X_{i}^{k+1} \cap a_{i}^{\prime 0} \cap$ $\ldots \cap a_{i}^{\prime k+1}$ is infinite for every $\left.i<n^{*}\right)$. Hence we get strictly increasing functions $g_{i}^{0}: \omega \rightarrow \omega$ for $i<n^{*}$ with $\left(g_{0}^{0}[\omega], \ldots, g_{n^{*}-1}^{0}[\omega]\right) \in\left([\omega]^{\omega}\right)^{n^{*}}$. As $\mathcal{A}$ is a maximal antichain there is a $\bar{c}^{0}=\left(c_{0}^{0}, \ldots, c_{n^{*}-1}^{0}\right) \in \mathcal{A}$ such that $\left(g_{0}^{0}[\omega], \ldots, g_{n^{*}-1}^{0}[\omega]\right)$ and $\bar{c}^{0}$ are compatible in $(\mathfrak{P}(\omega) / \text { fin })^{n^{*}}$. Define

$$
{ }^{\varepsilon} Y_{i}^{n}:= \begin{cases}{ }^{\alpha} X_{i}^{n} \backslash c_{i}^{0}, & \text { if } \varepsilon=0 \\ { }^{\alpha} X_{i}^{n}, & \text { if } \varepsilon=1\end{cases}
$$

Since we have $\left({ }^{\alpha} X_{0}^{n}, \ldots,{ }^{\alpha} X_{n^{*}-1}^{n}\right) \in \mathcal{J}(\mathcal{A})^{+}$for every $n \in \omega$ and of course $\left(c_{0}^{0}, \ldots, c_{n^{*}-1}^{0}\right)$ is not in $\mathcal{J}(\mathcal{A})^{+}$, for every $n \in \omega$ there exists a sequence $\left(\varepsilon_{0}^{n}, \ldots, \varepsilon_{n^{*}-1}^{n}\right) \in\{0,1\}^{n^{*}}$ such that $\varepsilon_{i}^{n}=0$ for at least one $i<n^{*}$ and we have $\left(\varepsilon_{0}^{n} Y_{0}^{n}, \ldots,{ }_{n^{*}-1}^{n} Y_{n^{*}-1}^{n}\right) \in \mathcal{J}(\mathcal{A})^{+}$. Because the sequences $\left({ }^{\alpha} X_{i}^{n}\right)_{n \in \omega}$ are decreasing we have the following: If $\left({ }^{\varepsilon_{0}} Y_{0}^{n}, \ldots,{ }^{\varepsilon_{n^{*}}-1} Y_{n^{*}-1}^{n}\right) \notin \mathcal{J}(\mathcal{A})^{+}$for some sequence $\left(\varepsilon_{0}, \ldots, \varepsilon_{n^{*}-1}\right) \in\{0,1\}^{n^{*}}$ then for every $m>n$ we have $\left({ }^{\varepsilon_{0}} Y_{0}^{m}, \ldots,{ }^{\varepsilon_{n^{*}-1}} Y_{n^{*}-1}^{m}\right) \notin$ $\mathcal{J}(\mathcal{A})^{+}$. So we can choose an $\bar{n} \in \omega$ large enough such that for every $m>\bar{n}$ we have $\varepsilon_{i}^{m}=1$ whenever $\varepsilon_{i}^{\bar{n}}=1$. Let $\varepsilon_{i}:=\varepsilon_{i}^{\bar{n}}$ for every $i<n^{*}$, then $\varepsilon_{i}=0$ for at least one $i<n^{*}$ and $\left({ }^{\varepsilon_{0}} Y_{0}^{n}, \ldots,{ }^{\varepsilon_{n^{*}-1}} Y_{n^{*}-1}^{n}\right) \in \mathcal{J}(\mathcal{A})^{+}$for every $n \in \omega$.

Let ${ }^{1} Z_{i}^{n}:={ }^{\varepsilon_{i}} Y_{i}^{n}$ for every $i<n^{*}$ and every $n \in \omega$. So we have an $n^{*}$-tupel ${ }^{1} \bar{Z}=\left(\left({ }^{1} Z_{0}^{n}\right)_{n \in \omega}, \ldots,\left({ }^{1} Z_{n^{*}-1}^{n}\right)_{n \in \omega}\right)$ of descending sequences in $[\omega]^{\omega}$ such that $\left({ }^{1} Z_{0}^{n}, \ldots,{ }^{1} Z_{n^{*}-1}^{n}\right) \in \mathcal{J}(\mathcal{A})^{+}$for every $n \in \omega$. Notice that we have $\Pi_{i<n^{*}}\left\{{ }^{1} Z_{i}^{n} \mid n \in\right.$ $\omega\} \subseteq \Pi_{i<n^{*}} \bigcup_{\nu<\alpha} F_{i}^{\nu}$.

Now we construct functions $g_{0}^{1}, \ldots, g_{n^{*}-1}^{1}: \omega \rightarrow \omega$ in the same way as before but for ${ }^{1} \bar{Z}$ instead of ${ }^{\alpha} \bar{X}$. Choose $g_{i}^{1}(0) \in{ }^{1} X_{i}^{0} \cap a_{i}^{\prime 0}$ for every $i<n^{*}$ and if $g_{0}^{1}(k), \ldots, g_{n^{*}-1}^{1}(k)$ are already chosen take for every $i<n^{*}$

$$
g_{i}^{1}(k+1) \in{ }^{1} Z_{i}^{0} \cap \ldots \cap{ }^{1} Z_{i}^{g_{i}^{1}(k)} \cap a_{i}^{\prime 0} \cap \ldots \cap a_{i}^{\prime k+1}
$$

with $g_{i}^{1}(k+1)>g_{i}^{1}(k)$. Then there exists an element $\bar{c}^{1} \in \mathcal{A}$ such that $\left(g_{0}^{1}[\omega], \ldots, g_{n^{*}-1}^{1}[\omega]\right)$ and $\bar{c}^{1}$ are compatible in $(\mathfrak{P}(\omega) / \text { fin })^{n^{*}}$. As there is at least one index $i<n^{*}$ with ${ }^{1} Z_{i}^{n}={ }^{\alpha} X_{i}^{n} \backslash c_{i}^{0}$ for every $n \in \omega$ the elements $\bar{c}^{0}$ and $\bar{c}^{1}$ are different. As before we let ${ }^{0} Y_{i}^{n}:={ }^{1} Z_{i}^{n} \backslash c_{i}^{1}$ and ${ }^{1} Y_{i}^{n}:={ }^{1} Z_{i}^{n}$ and we find a sequence $\left(\varepsilon_{0}, \ldots, \varepsilon_{n^{*}-1}\right) \in\{0,1\}^{n^{*}}$ with $\varepsilon_{i}=0$ for at least one $i<n^{*}$ such that $\left({ }^{\varepsilon} Y_{0}^{n}, \ldots,{ }_{n^{*}-1} Y_{n^{*}-1}^{n}\right) \in \mathcal{J}(\mathcal{A})^{+}$for every $n \in \omega$. For every $i<n^{*}$ and every $n \in \omega$ let ${ }^{2} Z_{i}^{n}:={ }^{\varepsilon_{i}} Y_{i}^{n}$.

Hence we can find a sequence $\left({ }^{l} \bar{Z}\right)_{l \in \omega}=\left(\left({ }^{l} Z_{0}^{n}\right)_{n \in \omega}, \ldots,\left({ }^{l} Z_{n^{*}-1}^{n}\right)_{n \in \omega}\right)$ of $n^{*}$-tupels of descending chains in $[\omega]^{\omega}$ with $\left({ }^{l} Z_{0}^{n}, \ldots,{ }^{l} Z_{n^{*}-1}^{n}\right) \in \mathcal{J}(\mathcal{A})^{+}$for every $n \in \omega$, a sequence $\left(\left(g_{0}^{l}, \ldots, g_{n^{*}-1}^{l}\right)\right)_{l \in \omega}$ of $n^{*}$-tupels of increasing functions from $\omega$ to $\omega$ with $g_{i}^{l}(k+1) \in Z_{i}^{l} g_{i}^{l}(k)$ and $\left(g_{0}^{l}[\omega], \ldots, g_{n^{*}-1}^{l}[\omega]\right) \in\left([\omega]^{\omega}\right)^{n^{*}}$ and a third sequence $\left(\bar{c}^{l}\right)_{l \in \omega}$ of pairwise different elements of $\mathcal{A}$ such that $\left|c_{i}^{l} \cap g_{i}^{l}[\omega]\right|=\omega$ for every $i<n^{*}$ and every $l \in \omega$.

Now we are ready to define diagonalizing functions $f_{0}, \ldots, f_{n^{*}-1}: \omega \rightarrow \omega$ for $\left({ }^{\alpha} X_{0}^{n}\right)_{n \in \omega}, \ldots,\left({ }^{\alpha} X_{n^{*}-1}^{n}\right)_{n \in \omega}$ such that $\left(f_{0}[\omega], \ldots, f_{n^{*}-1}[\omega]\right) \in \mathcal{J}(\mathcal{A})^{+}:$Choose 
$f_{i}(0) \in{ }^{\alpha} X_{i}^{0}$ for $i<n^{*}$. For $k>0$ there exists $j, l \in \omega$ such that $k=2^{j}(2 l+1)$. Choose for every $i<n^{*}$

$$
f_{i}(k) \in c_{i}^{j} \cap g_{i}^{j}[\omega] \cap{ }^{\alpha} X_{i}^{0} \cap \ldots \cap{ }^{\alpha} X_{i}^{f_{i}(k-1)} \cap a_{i}^{\prime 0} \cap \ldots \cap a_{i}^{\prime k-1}
$$

with $f_{i}(k)>f_{i}(k-1)$.

Now we define $a_{i}^{\alpha}:=f_{i}[\omega]$ for every $i<n^{*}$. Hence for every $i<n^{*}$ we have that $a_{i}^{\alpha}$ diagonalizes $\left({ }^{\alpha} X_{i}^{n}\right)_{n \in \omega}$; and as $\left(a_{0}^{\alpha}, \ldots, a_{n^{*}-1}^{\alpha}\right)$ cuts infinitly many $\bar{c}^{j}$ we have $\left(a_{0}^{\alpha}, \ldots, a_{n^{*}-1}^{\alpha}\right) \in \mathcal{J}(\mathcal{A})^{+}$and $\Pi_{i<n^{*}}\left\{a_{i}^{\nu} \mid \nu \leq \alpha\right\}$ has the finite intersection property in $\mathcal{J}(\mathcal{A})^{+}$.

Second case: $\Pi_{i<n^{*}}\left\{{ }^{\alpha} X_{i}^{n} \mid n \in \omega\right\}$ is not a subset of $\Pi_{i<n^{*}} \bigcup_{\nu<\alpha} F_{i}^{\nu}$. Then choose $a_{i}^{\alpha} \in[\omega]^{\omega}$ arbitrarily such that $a_{i}^{\alpha} \subseteq^{*} a_{i}^{\nu}$ for every $\nu<\alpha$ and every $i<n^{*}$.

This finishes our construction. Our construction guarantees that for every $i<n^{*}$ the set $\left\{a_{i}^{\alpha} \mid \alpha<\omega_{1}\right\}$ has the finite intersection property, thus we can define $U_{i}$ to be the filter generated by $\left\{a_{i}^{\alpha} \mid \alpha<\omega_{1}\right\}$. We have to check that $U_{i}$ is an ultrafilter, that this ultrafilter is Ramsey and that $U_{i}$ and $U_{j}$ for $i \neq j$ are not Rudin-Keisler-equivalent.

First we check that each $U_{i}$ is an ultrafilter: Suppose $a \in[\omega]^{\omega}$, so there exists $\alpha<\omega_{1}$ with $a=x_{\alpha}$. If $x_{\alpha} \notin U_{i}$ we have $a_{i}^{\alpha+1} \nsubseteq x_{\alpha}$, hence in the successor step we have chosen $a_{i}^{\alpha+1} \subseteq a_{i}^{\alpha} \backslash x_{\alpha} \subseteq \omega \backslash x_{\alpha}$, hence $\omega \backslash x_{\alpha} \in U_{i}$.

Check that each $U_{i}$ is Ramsey: Suppose $\left(X_{n}\right)_{n \in \omega}$ is a descending chain in $U_{i}$. Then there exists $\alpha \in \operatorname{Lim}\left(\omega_{1}\right)$ such that $\left(X_{n}\right)_{n \in \omega}=\left({ }^{\alpha} X_{i}^{n}\right)_{n \in \omega}$ and $\Pi_{i<n^{*}}\left\{{ }^{\alpha} X_{i}^{n} \mid n \in \omega\right\} \subseteq \Pi_{i<n^{*}} \bigcup_{\nu<\alpha} F_{i}^{\nu}$, hence we are in the first case of the limit step of the construction and $a_{i}^{\alpha} \in U_{i}$ diagonalizes $\left({ }^{\alpha} X_{i}^{n}\right)_{n \in \omega}$.

It remains to check that for $i, j<n^{*}$ with $i \neq j$ that the ultrafilters $U_{i}$ and $U_{j}$ are not Rudin-Keisler-equivalent: Suppose there exists a bijection $h: \omega \rightarrow \omega$ such that $U_{i}=\left\{h[x] \mid x \in U_{j}\right\}$. But there is an $\alpha<\omega_{1}$ with $h=h_{\alpha}$ and in the successor step of our construction we have chosen $a_{i}^{\alpha+1}$ and $a_{j}^{\alpha+1}$ such that $h_{\alpha}\left[a_{i}^{\alpha+1}\right] \cap a_{j}^{\alpha+1}=\emptyset$ or $h_{\alpha}\left[a_{j}^{\alpha+1}\right] \cap a_{i}^{\alpha+1}=\emptyset$, hence we get a contradiction.

\subsection{The proof of the maintheorem}

Proof of theorem 3.1.1: Suppose $n^{*} \in \omega \backslash\{0\}$ and $\mathcal{A} \subseteq(\mathfrak{P}(\omega) / \mathrm{fin})^{n^{*}}$ is an infinite maximal antichain. Since we can collapse $2^{\omega}$ to $\omega_{1}$ by a $\sigma$-closed forcing, hence without changing the set $\mathfrak{P}(\omega)$, we can assume without loss of generality that $\mathrm{CH}$ is true. By lemma 3.3.1 there are pairwise not Rudin-Keisler-equivalent Ramsey ultrafilters $U_{0}, \ldots, U_{n^{*}-1}$ such that $U_{0} \times \ldots \times U_{n^{*}-1} \subseteq \mathcal{J}(\mathcal{A})^{+}$.

Suppose $\mathcal{A}$ is analytical. Notice that in this case $\mathcal{J}(\mathcal{A})$ is analytical, too. Choose 
a countable elementary submodel $\mathcal{N}$ of $H_{\Theta}$ for a sufficiently large $\Theta$ such that $\mathcal{J}(\mathcal{A}), U_{0}, \ldots, U_{n^{*}-1}, \operatorname{code}(\mathcal{J}(\mathcal{A})) \in \mathcal{N}$, where $\operatorname{code}(\mathcal{J}(\mathcal{A}))$ is the $\Sigma_{1}^{1}$-code of the analytical set $\mathcal{J}(\mathcal{A})$. Consider the image of the Mostowski collapse $\varphi(\mathcal{N})=: \tilde{\mathcal{N}}$ of $\mathcal{N}$, hence $\tilde{\mathcal{N}}$ is transitive, $\mathcal{J}(\mathcal{A})$ is analytical in $\tilde{\mathcal{N}}$ by the same code as in $V$, $\tilde{U}_{i}:=\varphi\left(U_{i}\right)=U_{i} \cap \mathcal{N}$, hence $\tilde{U}_{i} \in \tilde{\mathcal{N}}$ for every $i<n^{*}$ and $Q\left(\tilde{U}_{0}, \ldots, \tilde{U}_{n^{*}-1}\right)=$ $Q\left(U_{0}, \ldots, U_{n^{*}-1}\right) \cap \mathcal{N}=Q\left(U_{0}, \ldots, U_{n^{*}-1}\right) \cap \tilde{\mathcal{N}}$, hence $Q\left(\tilde{U}_{0}, \ldots, \tilde{U}_{n^{*}-1}\right) \in \tilde{\mathcal{N}}$. Let $\left(\dot{g}_{0}, \ldots, \dot{g}_{n^{*}-1}\right)$ be a name for a $Q\left(\tilde{U}_{0}, \ldots, \tilde{U}_{n^{*}-1}\right)$-generic real over $\tilde{\mathcal{N}}$. By the pure decision property of $Q\left(\tilde{U}_{0}, \ldots, \tilde{U}_{n^{*}-1}\right)$ (fact 3.2.7) there exists $(\emptyset, S) \in$ $Q\left(\tilde{U}_{0}, \ldots, \tilde{U}_{n^{*}-1}\right)$ such that

$$
\text { (i) }(\emptyset, S) \Vdash_{Q\left(\tilde{U}_{0}, \ldots, \tilde{U}_{n^{*}-1}\right)}\left(\dot{g}_{0}, \ldots, \dot{g}_{n^{*}-1}\right) \in \mathcal{J}(\mathcal{A})
$$

or

(ii) $(\emptyset, S) \Vdash_{Q\left(\tilde{U}_{0}, \ldots, \tilde{U}_{n^{*}-1}\right)}\left(\dot{g}_{0}, \ldots, \dot{g}_{n^{*}-1}\right) \notin \mathcal{J}(\mathcal{A})$.

Claim: There exists $\bar{a} \in[\omega]^{\omega}$, which is $Q\left(\tilde{U}_{0}, \ldots, \tilde{U}_{n^{*}-1}\right)$-generic over $\tilde{\mathcal{N}}$, such that $a_{i} \subseteq S_{i}$ and $a_{i} \in U_{i}$ for every $i<n^{*}$.

Proof of the claim: Let $\left(O_{n}\right)_{n \in \omega}$ be an enumeration of all open and dense subsets of $Q\left(\tilde{U}_{0}, \ldots, \tilde{U}_{n^{*}-1}\right)$ in $\tilde{\mathcal{N}}$. Consider the following strategy for player $I$ in the game $G\left(\tilde{U}_{0}, \ldots, \tilde{U}_{n^{*}-1}\right)$ (defined in definition 3.2.3): In the beginning, player $I$ plays $\left(s^{0}, S^{0}\right) \in O_{0}$ with $\left(s^{0}, S^{0}\right) \leq(\emptyset, S)$. Player $I I$ answers with $k_{0} \in S_{0}^{0}$. In the $(m+1)$ th-move, player $I$ plays $\left(s^{m+1}, S^{m+1}\right) \in O_{m+1}$ with $\left(s^{m+1}, S^{m+1}\right) \leq\left(s^{m}, S^{m}\right)$ and additionally $k_{m} \in s^{m+1}$ (this is possible, since $O_{m+1}$ is open is dense). Player $I I$ responds with $k_{m+1} \in S_{(m+1) \bmod n^{*}}^{m+1}$. By lemma 3.2.4 player $I$ has no winning strategy, so the just defined one is not a winning one. Fix a play $\left(\left(s^{n}, S^{n}\right)_{n \in \omega},\left\{k_{n} \mid n \in \omega\right\}\right)$ such that player $I$ plays $\left(s^{n}, S^{n}\right)$ as his above defined strategy orders, player $I I$ plays $\left\{k_{n} \mid n \in \omega\right\}$ and player $I$ does not win. Hence we have $\left\{k_{n} \mid n \equiv i\left(\bmod n^{*}\right)\right\} \in U_{i}$ for every $i<n^{*}$. Define $\bar{a}:=\bigcup_{i \in \omega} s^{i}$ and $a_{i}:=\left\{k_{j} \mid j \equiv i\left(\bmod n^{*}\right)\right\}$, where $\left(k_{i}\right)_{i<\omega}$ is the increasing enumeration of $\bar{a}$. Clearly, we have $a_{i} \subseteq S_{i}$ and since $\left\{k_{n} \mid n \equiv i\left(\bmod n^{*}\right)\right\} \subseteq a_{i}$ we have $a_{i} \in U_{i}$ for every $i<n^{*}$. It remains to prove that $\bar{a}$ is $Q\left(\tilde{U}_{0}, \ldots, \tilde{U}_{n^{*}-1}\right)$-generic over $\tilde{\mathcal{N}}$. For this, by the remarks after the definition 3.2.1 of $Q$, we have to prove that for every open and dense set $O \subseteq Q\left(\tilde{U}_{0}, \ldots, \tilde{U}_{n^{*}-1}\right)$ with $O \in \tilde{\mathcal{N}}$ there exists $(t, T) \in O$ such that $t_{i} \subseteq a_{i}$ and $a_{i} \backslash t_{i} \subseteq T_{i}$ for every $i<n^{*}$. So suppose $O \in \tilde{\mathcal{N}}$ is open and dense in $Q\left(\tilde{U}_{0}, \ldots, \tilde{U}_{n^{*}-1}\right)$, hence $O=O_{n}$ for some $n \in \omega$. Consider $\left(s^{n}, S^{n}\right)$, the element of $O_{n}$ played by player $I$. Easily, we have $s_{i}^{n} \subseteq a_{i}$ and $a_{i} \backslash s_{i}^{n} \subseteq S_{i}^{n}$, as required.

$\square($ claim $)$

Now, if $(i)$ is true we have

$$
(i)^{\prime} \tilde{\mathcal{N}}[\bar{a}] \models\left(a_{0}, \ldots, a_{n^{*}-1}\right) \in \mathcal{J}(\mathcal{A})
$$


and if $(i i)$ is true we have

$$
(i i)^{\prime} \tilde{\mathcal{N}}[\bar{a}] \models\left(a_{0}, \ldots, a_{n^{*}-1}\right) \notin \mathcal{J}(\mathcal{A}) .
$$

But by fact 3.2.6 this implies

$$
(i)^{\prime \prime} \tilde{\mathcal{N}}[\bar{c}] \models\left(c_{0}, \ldots, c_{n^{*}-1}\right) \in \mathcal{J}(\mathcal{A})
$$

for every $\bar{c}$ with $c_{i} \in\left[a_{i}\right]^{\omega}$ for every $i<n^{*}$ or

$$
(i i)^{\prime \prime} \tilde{\mathcal{N}}[\bar{c}] \models\left(c_{0}, \ldots, c_{n^{*}-1}\right) \notin \mathcal{J}(\mathcal{A})
$$

for every $\bar{c}$ with $c_{i} \in\left[a_{i}\right]^{\omega}$ for every $i<n^{*}$. But $\Sigma_{1}^{1}$-definitions are absolute for transitive models, hence

$$
(i)^{\prime \prime \prime} V \models\left(c_{0}, \ldots, c_{n^{*}-1}\right) \in \mathcal{J}(\mathcal{A})
$$

for every $\bar{c}$ with $c_{i} \in\left[a_{i}\right]^{\omega}$ for every $i<n^{*}$ or

$$
(i i)^{\prime \prime \prime} V \models\left(c_{0}, \ldots, c_{n^{*}-1}\right) \notin \mathcal{J}(\mathcal{A})
$$

for every $\bar{c}$ with $c_{i} \in\left[a_{i}\right]^{\omega}$ for every $i<n^{*}$. But both is impossible. 


\section{Bibliography}

[BaJu] T. Bartoszynski, H. Judah, Set Theory. On the Structure of the Real line; A. K. Peters, Wellesley, Massachusetts (1995).

[Br] J. BREndLe, Combinatorial aspects of the meager and null ideals and of other ideals on the reals; Thesis for Habilitation, Eberhard-KarlsUniversität Tübingen, 1994/95.

[Go] M. Goldstern, Tools for your forcing construction; Set theory of the reals (Ramat Gan, 1991), Israel Mathematical Conference Prooceedings, vol. 6, Bar Ilan University, Ramat Gan, 1992, pp. 307-362.

[GoJSp] M. Goldstern, M. J. Johnson, O. Spinas, Towers on trees; Proceedings of the American Mathematical Society 122, vol. 2 (1994), pp. 557564 .

[GoReShSp] M. Goldstern, M. Repicky, S. Shelah, O. Spinas, On tree ideals; Proceedings of the American Mathematical Society 123, vol. 5 (1995), pp. 1573-1581.

[Je] T. JeCH, Set Theory; New York, Academic Press (1978).

[JoSp] S. Jossen, O. SpInas, A two-dimensional tree ideal; Berichtsreihe des Mathematischen Seminars Kiel, Vol. 00-32 (2000).

[JuMiSh] H. Judah, A. Miller, S. Shelah, Sacks forcing, Laver forcing, and Martin's axiom; Arch. Math. Logic 31, vol. 3 (1992), pp. 145-161.

[JuSh] H. Judah, S. Shelah, The Kunen-Miller chart; The Journal of Symbolic Logic, vol. 55 (1990), pp. 909-927.

[Ke] A. Kechris, On a notion of smallness for subsets of the Baire space; Transactions of the American Mathematical Society, vol. 229 (1977), pp. 191-207.

[Ko] S. Koppelberg, Handbook of Boolean algebras Vol. 1 (edited by J .D. Monk); North Holland, Amsterdam, New York, Oxford, 1989. 
[Ku] K. Kunen, Set Theory. An Introduction to Independence Proofs, North Holland, Amsterdam, New York, Oxford, 1980.

[LoShVe] A. Louveau, S. Shelah, B. Velickovic, Borel partitions of infinite subtrees of a perfect tree; Annals of Pure and Applied Logic 63, vol. 3 (1993), pp. 271-281.

[Ma] E. Marcewski, Sur une classe de fonctions de W. Sierpinśki et la classe correspondente d'ensembles; Fundamenta Mathematicae, vol. 24 (1935), pp. 17-37.

[Ma] A. R. D. Mathias, Happy families, Ann. Math. Logic 12 (1977), pp. 59111.

[Mi] A. MilleR, Rational perfect set forcing; Contemporary Mathematics, vol. 31 (1984), pp. 143-159.

[PiSz] Z. Piotrowski, A. Szymanski, Some remarks on category in topological spaces, Proceedings of the American Mathematical Society, vol. 101 (1987), pp. 156-160.

[Sh] S. Shelah, Proper forcing; Lecture Notes in Mathematics, vol. 940, Springer Verlag, Berlin, 1982.

[ShSp1] S. Shelah, O. Spinas, The distributivity numbers of $\mathfrak{P}(\omega) /$ fin and its spuares; Transactions of the American Mathematical Society 352, no. 5 (2000), pp. 2023-2047.

[ShSp2] S. Shelah, O. Spinas, The distributivity numbers of finite products of $\mathfrak{P}(\omega) /$ fin; Fundamenta Mathematicae 158 (1998), pp. 81-93.

[Sp1] O. SPInas, Generic trees; The Journal of Symbolic Logic, vol. 60 (1995), pp. 705-726.

[Sp2] O. SpInas, Ramsey and freeness properties of Polish planes; Proceedings of the London Mathematical Society, vol. 82 (2001), pp. 31-63.

[Sp3] O, SPINAS, Canonical behaviour of Borel functions on superperfect rectangles; J. Math. Logic 1, vol. 2 (2001), pp. 173-220.

[Sp4] O. SpInas, Partitioning products of $\mathfrak{P}(\omega) /$ fin; Pacific Journal of Mathematics, vol. 176, no. 1 (1996), pp. 249-262.

[Ve] B. Velickovic, ccc posets of perfect trees; Compositio Math. 79 (1991), pp. 279-294. 
